\title{
4G LTE NETWORK DATA COLLECTION AND ANALYSIS ALONG PUBLIC TRANSPORTATION ROUTES
}

by

Habiba Elsherbiny

A thesis submitted to the Department of Electrical and Computer Engineering

In conformity with the requirements for

the degree of Master of Applied Science

\author{
Queen's University \\ Kingston, Ontario, Canada
}

May, 2020

Copyright (C) Habiba Elsherbiny, 2020 


\begin{abstract}
With the advancements in wireless network technologies over the past few decades and the deployment of 4G LTE networks, the capabilities and services provided to end-users have become seemingly endless. Users of smartphones utilize high-speed network services while commuting on public buses and hope to have a consistent, high-quality connection for the duration of their trip. Due to the massive load demand on cellular networks and frequent changes in the underlying radio channel, users often experience sudden unexpected variations in the connection quality. To overcome such a variation and maintain a consistent connection, we need to predict these variations before they occur. This can be accomplished by analyzing different network quality parameters at various times and locations and investigating the main factors that affect the network's performance and network QoS.

To this end, we conducted a network survey via Kingston Transit, in Kingston, Ontario, using the Android network monitoring application G-NetTrack Pro from which we constructed a dataset of various client-side wireless network quality parameters. The dataset consists of 30 repeated public transit bus trips, each lasting no more than one hour. We studied two techniques for throughput analysis: regression predictive modelling and time series forecasting. For regression predictive modelling, we deployed various machine learning models on the collected data for throughput prediction and achieved the highest prediction performance with the random forest model. For time series forecasting, we used statistical methods as well as deep learning architectures. Our evaluation shows that the machine learning models had a higher throughput prediction performance than the time series forecasting techniques.

In this thesis, we present an analysis of the collected data, where we investigate the effects of time and location on the network's measured throughput and signal strength. Also, we discuss and compare the results of applying different throughput prediction techniques on the collected data.
\end{abstract}




\section{Acknowledgements}

I would like to express my sincere gratitude to my supervisor Prof. Hossam Hassanein for his endless guidance, support and constant feedback throughout this research work. Also, I would like to extend my appreciation to my co-supervisor Prof. Aboelmagd Noureldin for his valuable suggestions and insights during the planning and development of this research work.

I am deeply grateful to Prof. Hazem Abbas for his guidance and motivation, and for giving his time so generously to help me complete this research work.

I also wish to thank Basia Palmer for her patient and accurate review of this thesis and her useful suggestions.

I would like to thank all my colleagues in Queen's Telecommunications Research Lab. In particular, I would like to thank Ahmad Nagib for his generous help and support, Basma, Rawan, Sara and Mary for their continuous encouragement.

To my parents, Ashraf and Amany, thank you for always believing in me in every step of my life. Words can never describe how grateful I am to your endless love and support. I would like to extend my appreciation to my brothers, Ahmad and Amr, who have always stood by my side and encouraged me through hard times.

Last but not least, I wish to thank my Fiancé, Marwan, for being patient and supportive during my studies and for always encouraging me to do better. 


\section{Table of Contents}

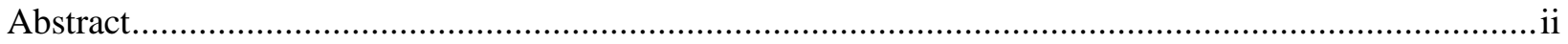

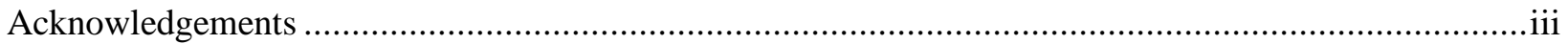

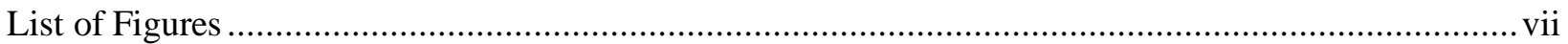

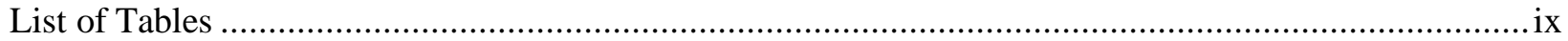

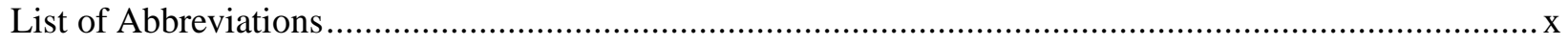

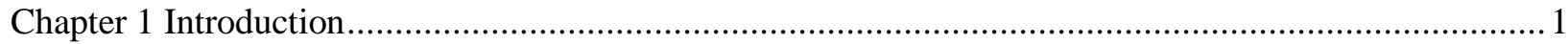

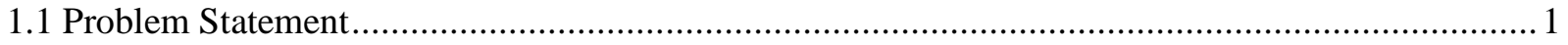

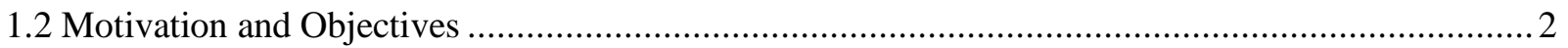

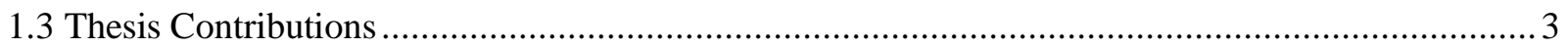

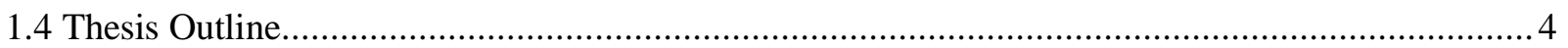

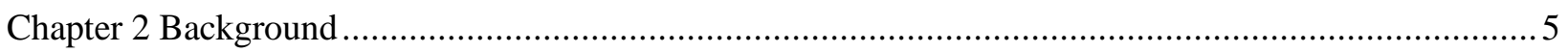

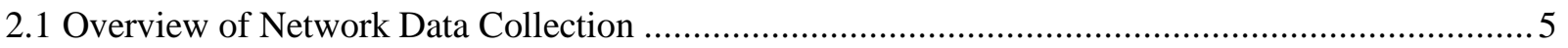

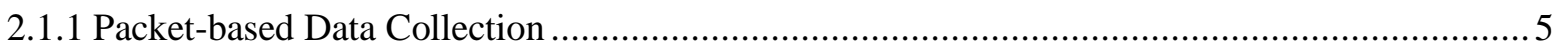

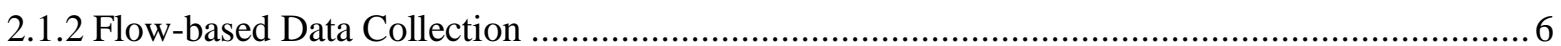

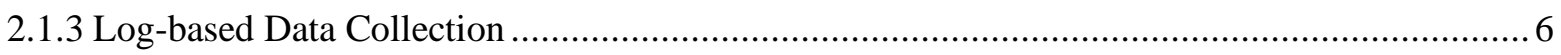

2.1.4 Data Collection Tools and Techniques................................................................................. 6

2.2 LTE Networks Performance Metrics .................................................................................. 7

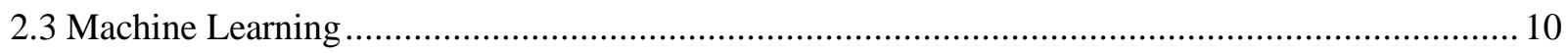

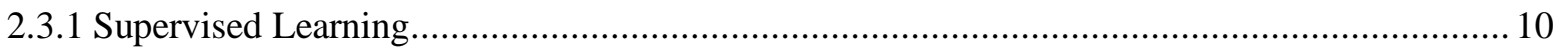

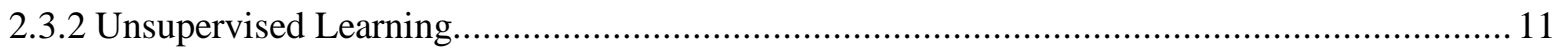

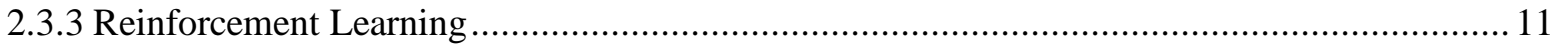

2.4 Overview of Throughput Prediction ............................................................................... 11

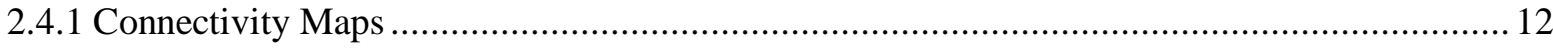

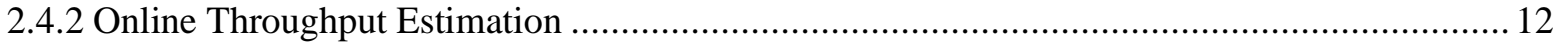

2.5 Related Work

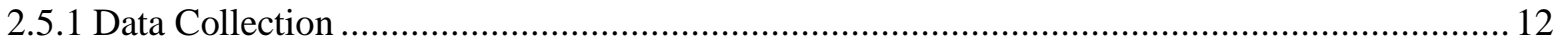

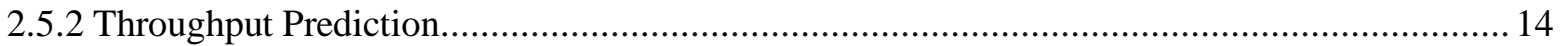

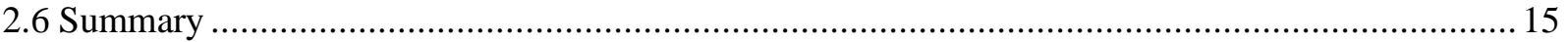

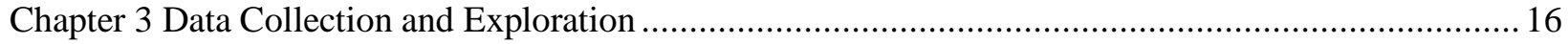

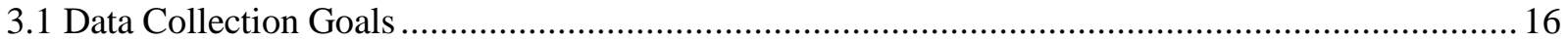

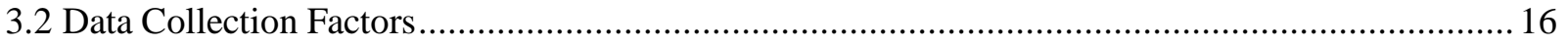

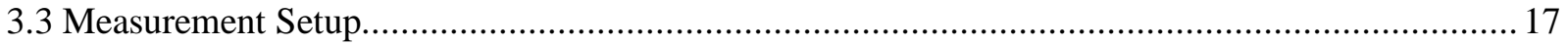




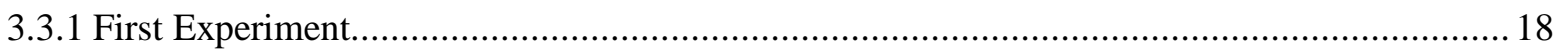

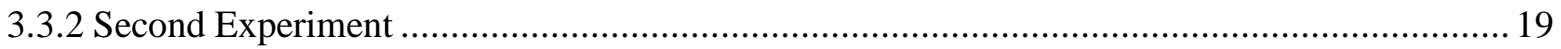

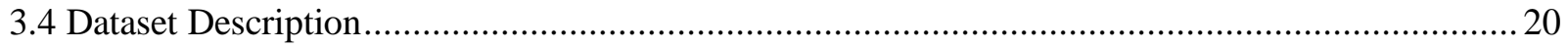

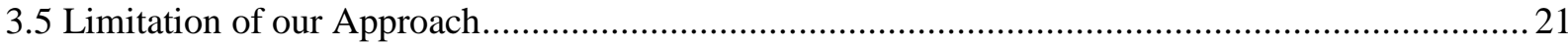

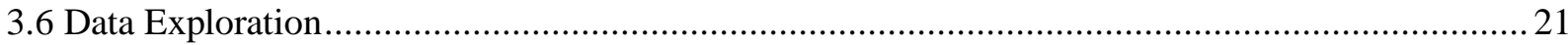

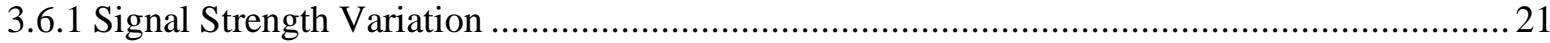

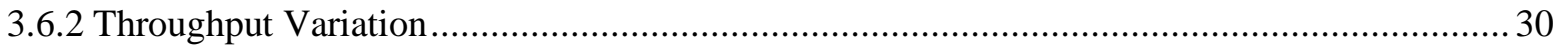

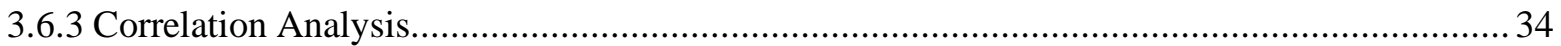

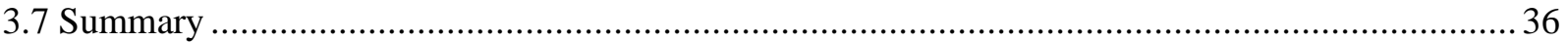

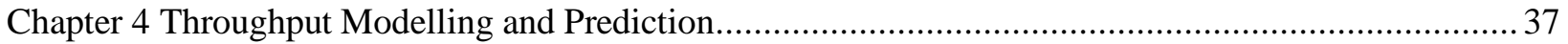

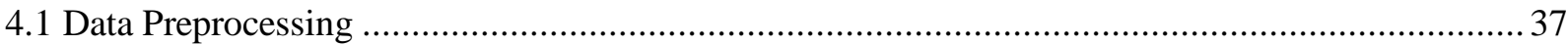

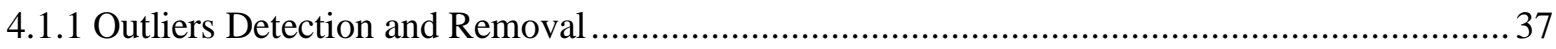

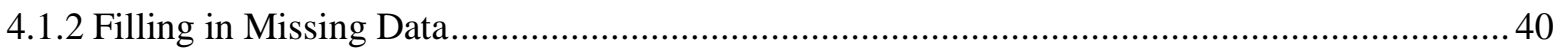

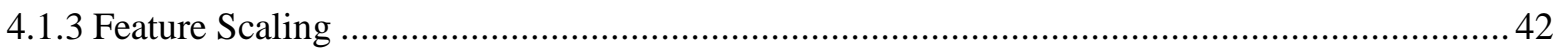

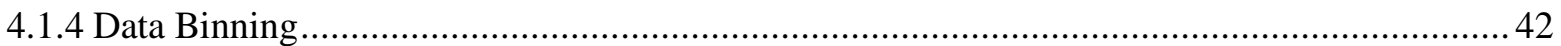

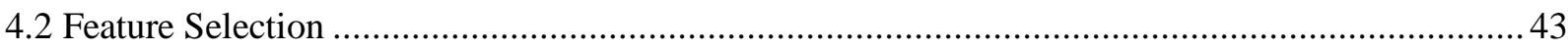

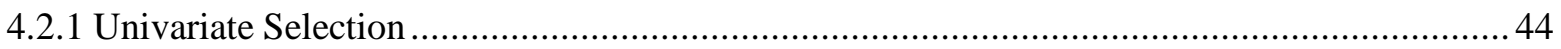

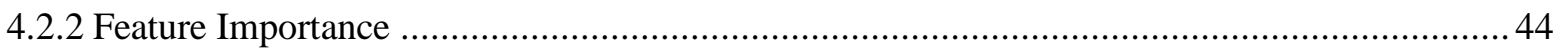

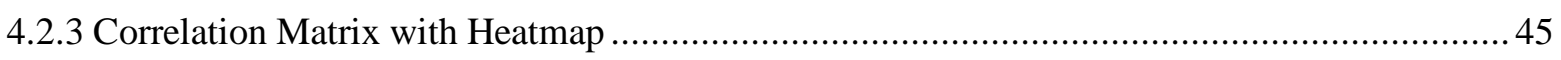

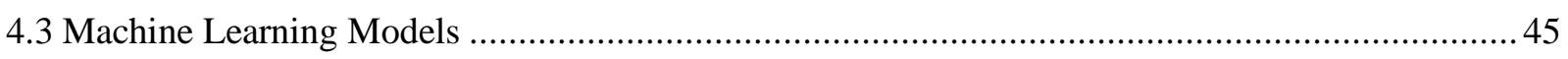

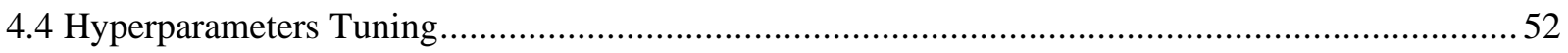

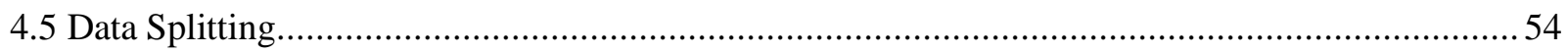

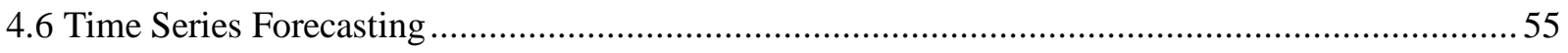

4.6.1 Autoregressive Integrated Moving Average (ARIMA)..................................................56

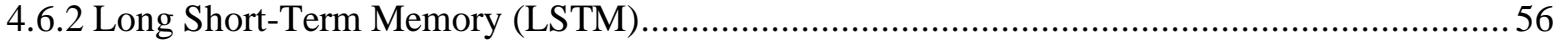

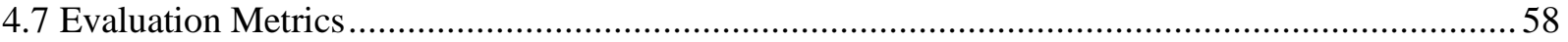

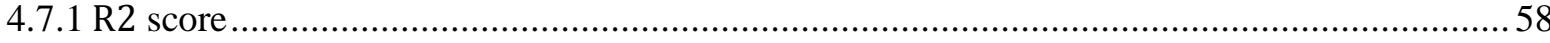

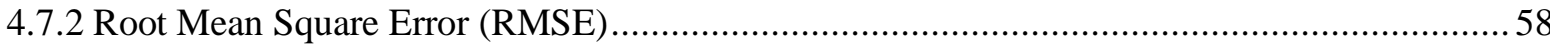

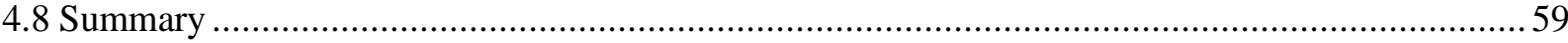

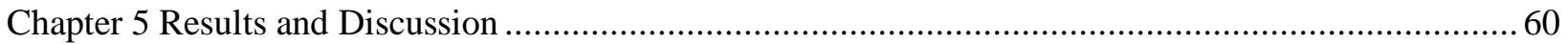

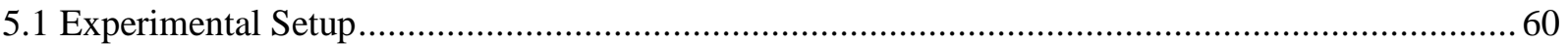

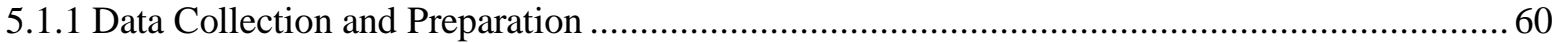

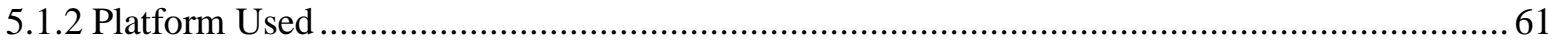

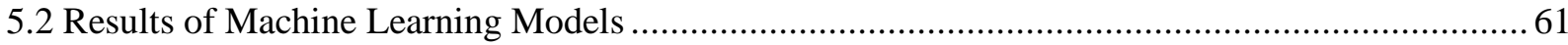




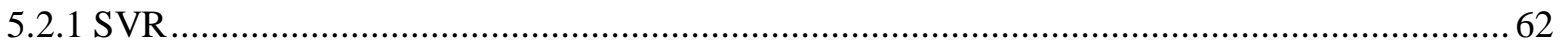

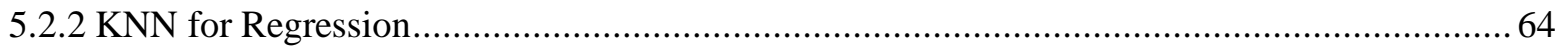

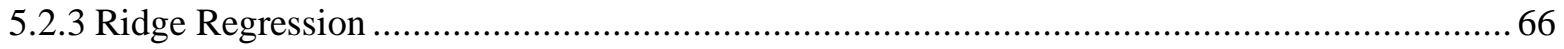

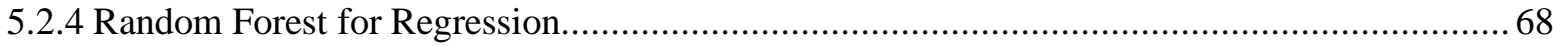

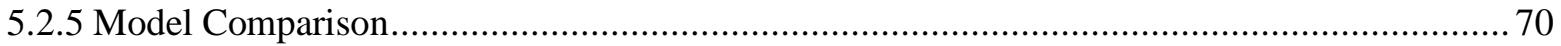

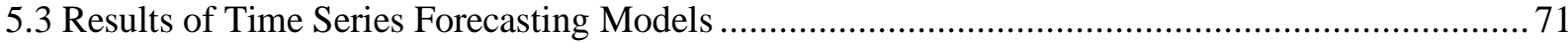

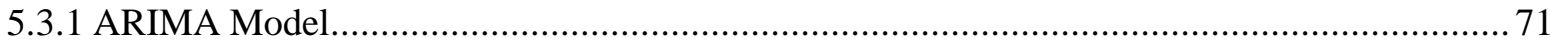

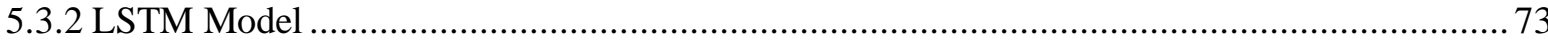

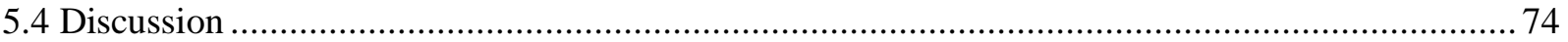

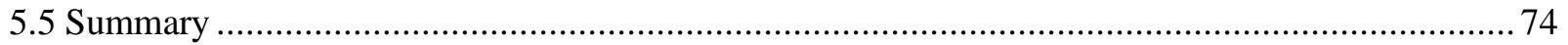

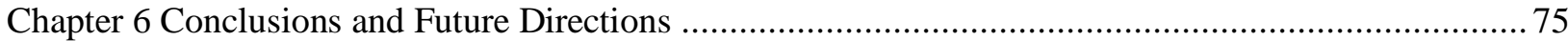

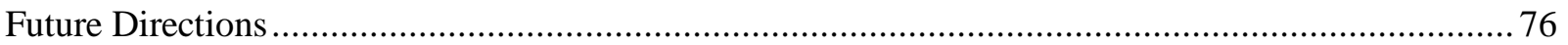

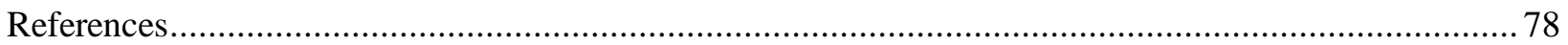




\section{List of Figures}

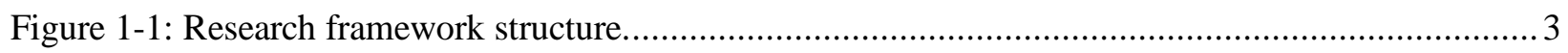

Figure 3-1: The $23.64 \mathrm{~km}$ trajectory of the Kingston Transit Express Bus 502. .................................... 18

Figure 3-2: Sample trip trajectory showing incomplete GPS recordings. .......................................... 19

Figure 3-3: Sample 9-am-trips (a) latitude and (b) signal strength variation per second........................ 22

Figure 3-4: Sample 12-pm-trips (a) latitude and (b) signal strength variation per second. ....................23

Figure 3-5: Sample 6-pm-trips (a) latitude and (b) signal strength variation per second....................... 24

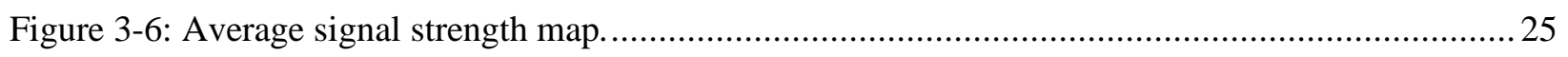

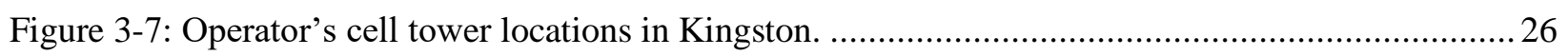

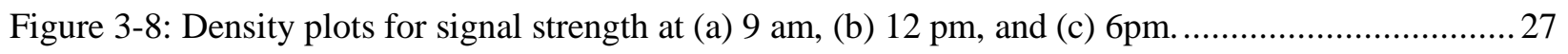

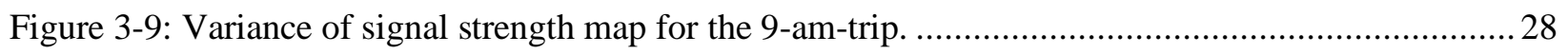

Figure 3-10: Variance of signal strength map for the 12-pm-trip. .....................................................29

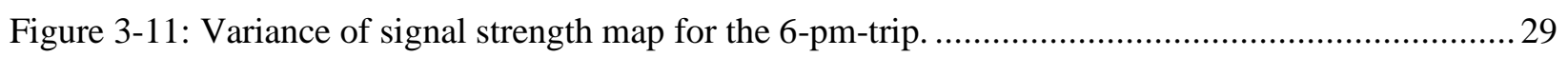

Figure 3-12: Throughput variation per second for sample bus 9 -am trips.......................................... 30

Figure 3-13: Throughput variation per second for sample 12-pm-trips.............................................. 31

Figure 3-14: Throughput variation per second for sample 6-pm-trips. .............................................. 31

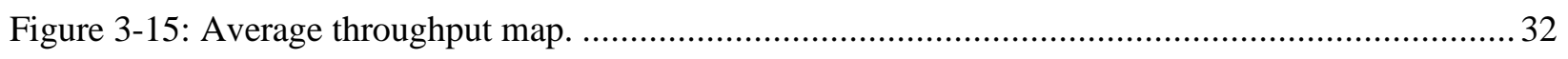

Figure 3-16: : Density plots for throughput at (a) 9 am, (b) 12 pm, and (c) 6pm............................... 33

Figure 3-17: Correlation matrix of different features in the dataset.................................................. 35

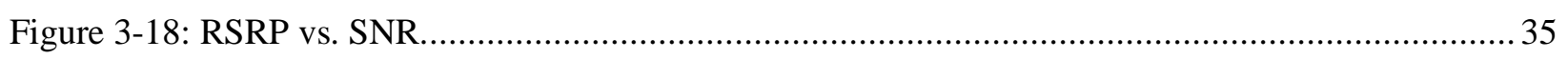

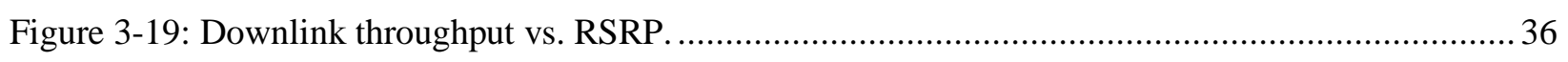

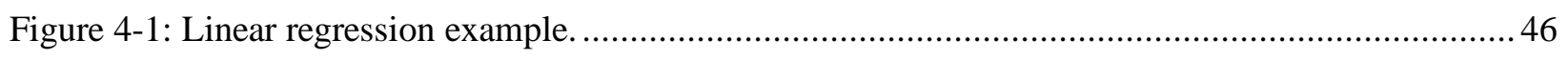

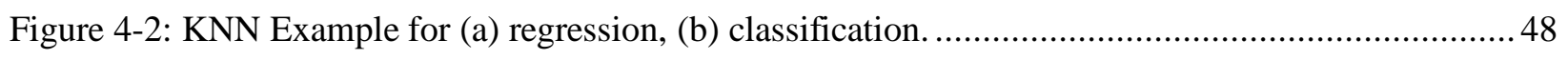

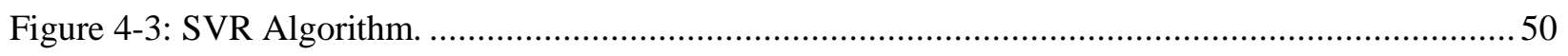

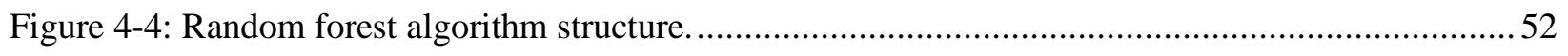

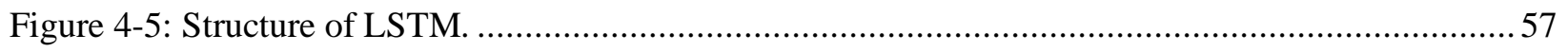

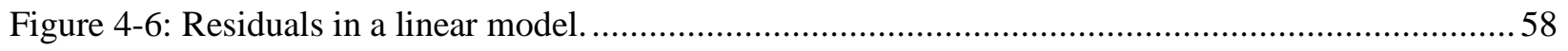

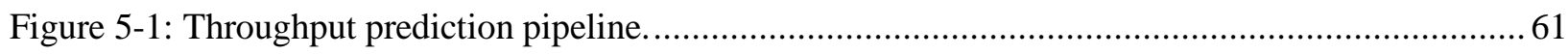

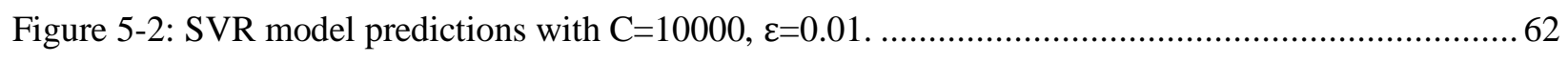

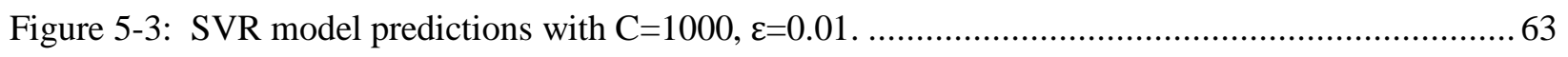

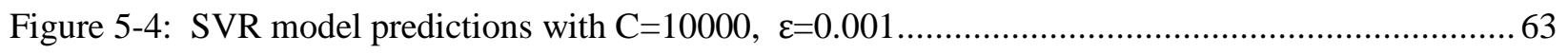

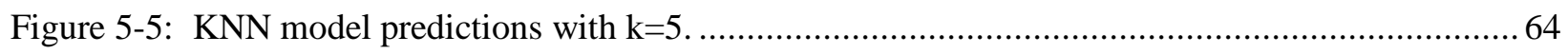




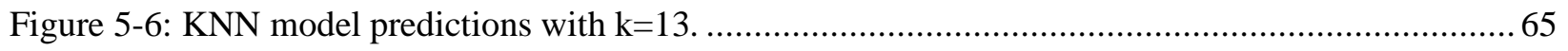

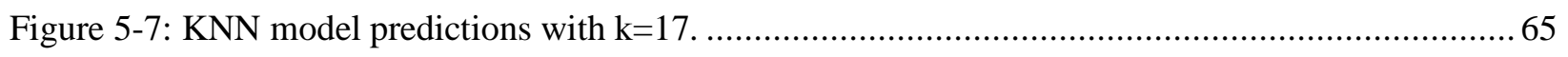

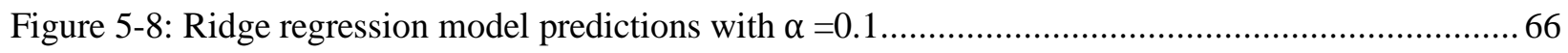

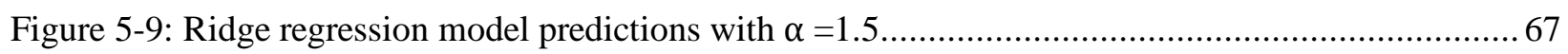

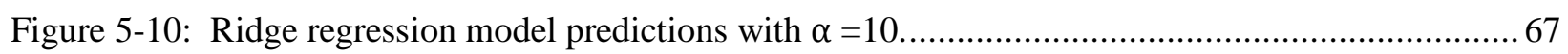

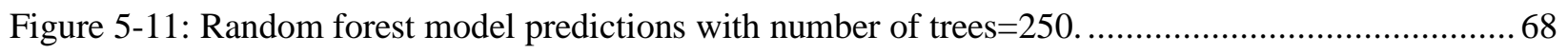

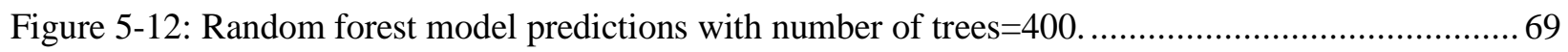

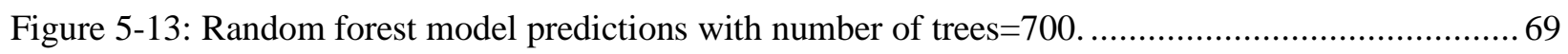

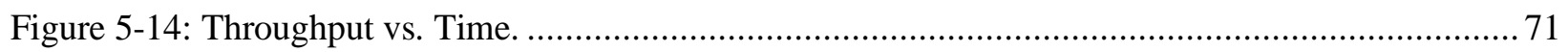

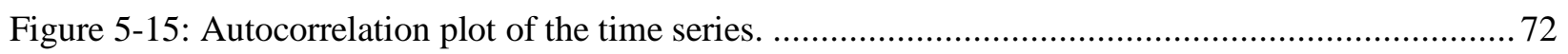

Figure 5-16: ARIMA model performance for time series throughput forecasting................................ 72

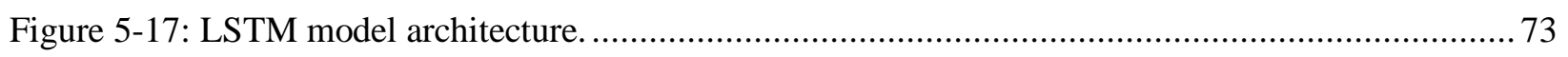

Figure 5-18: LSTM model performance for time series throughput forecasting. ................................ 73 


\section{List of Tables}

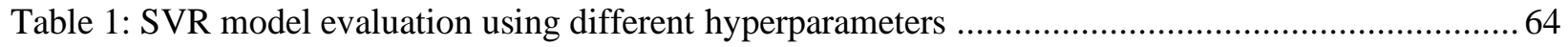

Table 2: KNN model evaluation using different hyperparameters................................................... 66

Table 3: Ridge regression model evaluation using different hyperparameters ......................................68

Table 4: Random forest model evaluation using different hyperparameters ....................................... 70

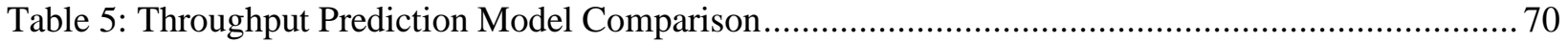




\section{List of Abbreviations}

\begin{tabular}{|c|c|}
\hline LTE & Long Term Evolution \\
\hline RRM & Radio Resource Management \\
\hline RSSI & Received Signal Strength Indicator \\
\hline RSRP & Reference Signal Received Power \\
\hline RSRQ & Reference Signal Received Quality \\
\hline CQI & Channel Quality Indicator \\
\hline SNR & Signal-to-noise Ratio \\
\hline QoS & Quality of Service \\
\hline GPS & Global Positioning Service \\
\hline IP & Internet Protocol \\
\hline QXDM & Qualcomm Extensible Diagnostic Monitor \\
\hline API & Application Program Interface \\
\hline 3GPP & 3rd Generation Partnership Project \\
\hline RTT & Round-Trip Time \\
\hline $\mathrm{ABR}$ & Adaptive bitrate Streaming \\
\hline UDP & User Datagram Protocol \\
\hline TCP & Transmission Control Protocol \\
\hline HSPA+ & High Speed Packet Access \\
\hline HSDPA & High Speed Downlink Packet Access \\
\hline WiFi & Wireless Fidelity \\
\hline IQR & Interquartile Range \\
\hline DBSCAN & Density-based Spatial Clustering of Applications with Noise \\
\hline MICE & Multivariate Imputation by Chained Equations \\
\hline
\end{tabular}




$\begin{array}{ll}\text { SVM } & \text { Support Vector Machines } \\ \text { SVR } & \text { Support Vector Regression } \\ \text { SVC } & \text { Support Vector Classification } \\ \text { KNN } & \text { K-Nearest Neighbors } \\ \text { MSE } & \text { Mean Squared Error } \\ \text { RMSE } & \text { Root Mean Square Error } \\ \text { RAM } & \text { Random Access Memory } \\ \text { RNN } & \text { Recurrent Neural Networks } \\ \text { LSTM } & \text { Long Short-Term Memory } \\ \text { ARIMA } & \text { Autoregressive Integrated Moving Average }\end{array}$




\section{Chapter 1}

\section{Introduction}

The past decade has witnessed a staggering evolution in cellular networks. Mobile wireless technologies have undergone four distinct generations. From uncomplicated voice calls in the first generation to highspeed, low latency and video streaming in the fourth generation. The increasing demand in network usage has proven the necessity of further service enhancements. However, these enhancements were faced with several obstacles. In this chapter, we shed light on the problems that prevent such improvements in cellular networks and present our solutions to these problems.

\subsection{Problem Statement}

Over the years, there has been a dramatic increase in mobile network traffic. The technological advancements in the 4G LTE networks have brought broadband speeds directly to smartphones, allowing mobile users to access high-speed internet services such as online gaming and video streaming while on a public transit bus. This has significantly increased the load on cellular networks, causing fluctuating loads on the network traffic. To cope with this increasing demand, cellular network operators are constantly looking ways to fulfill the client's expectations. However, this task has many challenges; it requires successful scheduling, network load balancing, and resource allocation. While taking into consideration cell tower locations, network quality and time of day, the problem can be broken down to three main aspects: the user's location, the achieved network quality and the time of the day.

In the past few years, these three aspects have been studied extensively in the literature. The researchers have taken different approaches to tackle the issues attached to these aspects. The most common issue faced in the related work was the sparsity of cellular network data. Consequently, the usage of modern network quality prediction techniques was limited, as most mechanisms require large amounts of data. To solve this problem, researchers directed their efforts towards collecting their own data. 
However, most of the data collection and analysis performed in the related work focused on $3 \mathrm{G}$ networks $[1,2,3]$. In addition to the lack of $4 \mathrm{G}$ LTE networks analysis, most of the research conducted did not investigate connectivity issues on public transportation.

We conducted a network survey along a $23.4 \mathrm{~km}$ public transit bus route covering both urban and suburban areas in Kingston, Ontario. To consider the effect of time and road traffic conditions, we conducted the measurements at three different times of the weekdays. To consider changing connectivity, we performed a comprehensive analysis on the collected data and applied machine learning algorithms, as well as time series forecasting approaches, for throughput prediction and forecasting ${ }^{1}$. Since throughput is a major indicator of the network's performance, the work done in the field utilized throughput prediction as one of the means of ensuring a high network quality.

\subsection{Motivation and Objectives}

The motivation for this research is the need to predict the network throughput based on other network parameters. This is an initial step that is required before tasks such as predictive resource allocation could be carried out by network providers. It is also a crucial step for maintaining the network QoS.

For the following reasons, public transportation vehicles are attractive candidates for cellular network analysis. Public transportation passengers generate massive amounts of mobile traffic, due to their intensive consumption of data. The routes and stops are known in advance, which allowed us to observe the status of the traffic during the bus trips and note any correlation with network quality of service (QoS).

To perform a precise analysis of 4G LTE networks availability within public transit buses, accurate and reliable data is a necessity. As there is no publicly available dataset of 4G LTE network parameters in Kingston, conducting a network survey of 4G LTE network data was required for this research.

\footnotetext{
${ }^{1}$ The main difference between prediction and forecasting is that forecasting takes into consideration the time dimension.
} 
The work presented in this thesis targets the following objectives:

- Collecting cellular network data containing measurements of various network performance indicators, throughput, and context information such as GPS location and bus speed.

- Analyzing the cellular connectivity levels on public transportation across Kingston.

- Investigating the correlation between signal strength and throughput.

- Applying throughput prediction algorithms on the collected data.

To the best of our knowledge, this is the first extensive analysis to be carried out over 4G LTE networks along public transportation in Kingston. The main stages of work are illustrated in Figure 1-1.

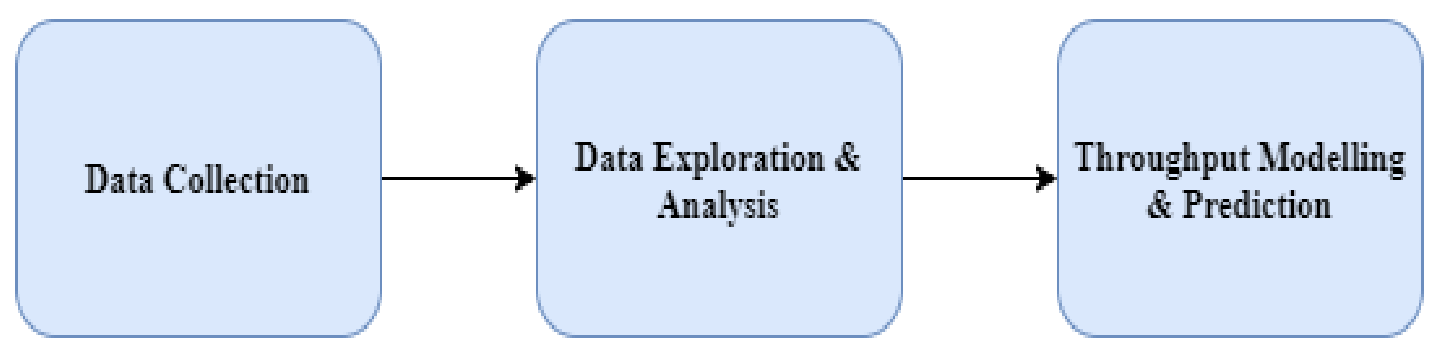

Figure 1-1: Research framework structure.

\subsection{Thesis Contributions}

The following are the key contributions of our research:

\section{1- Data Collection}

We constructed a 4G LTE dataset of wireless network parameters including signal strength measurements, downlink and uplink throughput measurements, and GPS locations.

We achieved this by conducting a network survey along a public bus route in Kingston Ontario for over 30 hours, covering a total of $700 \mathrm{~km}$. We used two different android smartphones for data collection. We noted the effects of time and road traffic by performing our measurements three times a day, for 10 days. To the best of our knowledge, this is the first 4G LTE dataset collected along public transportation routes in Kingston, Ontario. 


\section{2- Data Analysis}

We performed a thorough analysis on the collected measurements, and investigated the effects of time, location, road traffic condition and number of passengers on the signal strength and throughput measurements. Moreover, we studied the variations of the signal strength and throughput values at different times of the day and analyzed the relationship between the different wireless network parameters and the throughput.

\section{3- Throughput Modelling and Prediction}

To analyze the fluctuations in the network throughput measurements and anticipate them, we trained various machine learning models for throughput prediction using the collected dataset. We evaluated the models' performance on test data from our measurements using multiple evaluation metrics. Additionally, we performed a comparative analysis of the various models used.

\subsection{Thesis Outline}

The thesis is organized into six chapters. Chapter 1 provides an introduction, Chapter 2 gives an overview about data collection mechanisms and techniques, throughput prediction methods, and presents an extensive literature review. In Chapter 3, we explain our data collection approach and perform an analysis on the collected data. Chapter 4 presents the pipeline for the throughput prediction models that we applied to the data, as well as the preprocessing steps. Chapter 5 discusses and compares the experimental results of the different models we used for throughput prediction. Lastly, Chapter 6 summarizes our findings, and presents an insight regarding potential future research directions. 


\section{Chapter 2}

\section{Background}

In this chapter, we present background information about the topics that will be discussed throughout this thesis. In Section 2.1, we provide an overview about network data collection, explaining the different tools and techniques that are commonly used for data collection. In Section 2.2, we discuss the main metrics used for measuring network performance. In Section 2.3, we give a brief overview about the different types of machine learning algorithms. In Section 2.4, we talk about two widespread techniques that are used for throughput prediction. Finally, in Section 2.5, we discuss the related work conducted in the fields of data collection and throughput prediction.

\subsection{Overview of Network Data Collection}

Over the years, there has been a growing interest in collecting and analyzing network data for numerous reasons, such as network performance evaluation, resource allocation, traffic prediction, throughput prediction, security analysis and intrusion detection. Accordingly, researchers have proposed and studied various data collection mechanisms and techniques. In this section, we present three common mechanisms for data network collection: packet-based data collection, flow-based data collection and log-based data collection [4]. In addition, we describe the different tools and methods for collecting network data.

\subsubsection{Packet-based Data Collection}

In packet switched networks, the data is divided and encoded into packets. A source node typically sends packets to a destination node. When the destination node receives the packets, it decodes them to retrieve the data [4].

A common method for data collection in networks is packet capturing, which is the process of intercepting a data packet that is passing through the data network. Packet capturing can be done by a packet analyzer, also known as packet sniffer [5]. Packet capturing can be classified into two methods, active data collection 
methods and passive collection methods. Active data collection methods work by injecting test data into the network traffic and waiting for a response and then measuring the network performance parameters. Passive data collection methods work by monitoring the network traffic using a monitoring tool [4]. The monitoring tool is polled periodically, and information about the network performance is obtained.

\subsubsection{Flow-based Data Collection}

Another traditional method for network data collection is the flow-based collection. A network flow is made up of a set of packets having the same features. Flow based data collection involves monitoring the network flow at a certain location in the network. Any location can be used for flow monitoring, but the most effective location is at a core network device. Flow monitoring methods are often based on five tuples: the source IP address, the destination IP address, the source port, the destination port, and protocol type [4].

\subsubsection{Log-based Data Collection}

Log-based data collection works by monitoring the network log files, which are files that store records of network events. Different network devices including hosts, mobile devices, routers, and data centers contain $\log$ files. In log-based data collection, the network logs are first probed, and data is acquired from them. Then, the data is parsed to extract important features from it. Finally, analysis is performed on the data, often using pattern matching or machine learning approaches [4].

\subsubsection{Data Collection Tools and Techniques}

Several tools and techniques have been proposed for network data collection. Each tool has different characteristics and choosing the right one depends on factors, such as the nature of the problem and the budget. The following are three commonly used tools for network data collection:

\section{1- Qualcomm eXtensible Diagnostic Monitor (QXDM)}

QXDM is a tool developed by Qualcomm that can record various radio information in real time for phones with Qualcomm chipsets [6]. To record data, a phone is connected using a USB cable to a laptop that runs QXDM. The tool then communicates with the phone and records the traces containing radio 
information [7]. An advantage of using this tool is that it can collect data with a granularity of milliseconds. However, it requires expensive licenses and might not be easily deployed on low cost hardware.

\section{2- Android Smartphone}

Android smartphones rely on the public Android API for obtaining information about various network quality parameters. This approach is simple, inexpensive and can be used out-of-the-box. However, the problem with this approach is that it has a maximum granularity of one second, which is less than that of QXDM.

\section{3- Crowdsourcing}

Crowdsourcing is the process of using individuals or organizations to obtain goods and services [8]. In crowdsourcing, the work is divided among participants who work together toward a common goal. In the context of network data collection, crowdsourcing allows collecting massive amounts of data with the help of volunteers who simply install an application on their devices and use it for conducting measurements. This approach allows for more scenarios for collection but requires multiple volunteers. Moreover, the more diversity might cause the data to have a high variance.

\subsection{LTE Networks Performance Metrics}

LTE is the 3GPP-standardized wireless cellular network that focuses on providing very high data rates, high spectral efficiency, improved capacity and coverage, short round trip time, and spectrum flexibility [9]. To assess the performance of LTE, several metrics should be taken into consideration. The following are the main metrics that determine the performance and quality of the LTE networks:

\section{1- Radio Resource Management Measurements}

Radio Resource Management (RRM) is the system level management of radio transmission characteristics in wireless communication systems [10]. RRM provides a set of measurements to ensure the quality of the LTE networks. The RRM measurements are as follows: 
- Received Signal Strength Indicator (RSSI): RSSI is an estimated measure of the total power in a radio signal received by a client device, including the interference from neighboring cells and other sources, and is measured in $\mathrm{dB}[11]$. There are four main parameters related to the RSSI: dynamic range, accuracy, linearity and average period [12]. The RSSI dynamic range states the maximum and minimum received signal energy that the receiver can measure. The RSSI accuracy defines the average error for each RSSI measurement, and the RSSI linearity specifies how the RSSI plot deviates from a straight line versus the actual received input signal power. The average period indicates the period of time during which the signal strength is measured and then averaged to produce the RSSI value.

- Reference Signal Received Power (RSRP): RSRP is a cell-specific signal strength measurement that indicates the power of the LTE reference signals over the bandwidth and narrowband and is expressed in $\mathrm{dBm}$. It is used for ranking different cells based on their signal strength and for making handover or cell reselection decisions. Handover occurs when the RSRP value of the serving cell falls below that of the neighboring cell by a predefined threshold for a certain period of time [12]. RSRP values range from $-140 \mathrm{dBm}$ to $-44 \mathrm{dBm}$, where a larger value indicates a higher signal strength and likewise a lower value indicates a lower signal strength.

- Reference Signal Received Quality (RSRQ): RSRQ is a cell-specific measurement that indicates the quality of the received reference signal and is expressed in $\mathrm{dB}$. When the value of the RSRP is not enough to make reliable handover or cell reselection decisions, the value of the RSRQ is used to give more information. The value of the RSRQ depends on the RSSI and the number of used resource blocks, and is computed by:

$$
R S R Q=\frac{(N * R S R P)}{R S S I}
$$

where $\mathrm{N}$ is the number of resource blocks. The range of the RSRQ is defined from $-19.5 \mathrm{~dB}$ to -3 $\mathrm{dB}$, where the larger value indicates a higher signal quality and the smaller a lower signal quality. 
- Channel Quality Indicator (CQI): CQI is a 4-bit integer that is sent from user equipment (UE) to the eNodeB, which is the base station responsible for radio communications, resource management and message scheduling in LTE networks. The eNodeB uses the CQI to indicate a suitable downlink transmission data rate in order to ensure a stable connection between the network and the UE. The CQI values range from one to 31 , where larger values indicate a higher quality and smaller values indicate a lower quality [11]. Furthermore, CQI is a quantized and scaled version of the experienced SINR [9]. SNR/SINR stands for signal-to-noise ratio/ signal-to-interferenceplus-noise ratio and is defined as the ratio of the signal power to noise power, where the noise is the sum of the interference power and background noise power.

\section{2- Throughput}

Throughput is a measure of the network's actual data transmission rate [12]. Particularly, it measures the percentage of messages or data packets that are successfully delivered over a communication channel in a given time period. Throughput is measured in bits per second (bps) and can be divided into downlink throughput and uplink throughput. Downlink throughput indicates the number of packets successfully delivered in the downlink channel per unit time, while uplink throughput refers to the number of packets successfully delivered in the uplink channel per unit time. Throughput can be further classified into user throughput and average cell throughput. User throughput is a measure of the average amount of data being received by a certain user in the network and average cell throughput is a measure of the total throughput of all the users in the network.

In our work, we collected user throughput measurements and employed machine learning models to predict the user throughput based on other network parameters. Thus, for the remainder of this thesis, throughput will always refer to the user throughput.

\section{3- Latency}

Latency is a measure of how long it takes a data packet to travel from the source node in the network to the destination node. Network latency is often measured by the round-trip time (RTT), which is the 
time taken by a data packet to travel to a destination node and for an acknowledgment to be sent back to the source node and is expressed in milliseconds (ms).

There are multiple factors that affect the network latency; the following are the more common factors:

- Packet Size: The time taken to send a large packet would be more than the time taken to send a small packet.

- Packet Loss: Packet loss is the percentage of packets that get lost on the way from the source node to the destination node, and therefore are not received in the destination node.

- Signal Strength: Signal strength affects the network latency; a weak signal could cause a high latency.

- Transmission Media: The type of medium used to transmit the data packets can impact the network latency, as some mediums have higher latency than others. For example, old copper cables would have higher latency than fiber-optic cables [13].

\subsection{Machine Learning}

Machine learning has been shown to be successful in various fields, such as: computer vision, speech recognition and natural language processing. Machine learning algorithms build a mathematical model of training data in order to make predictions or decisions without being explicitly programmed to perform the task [14]. Generally, machine learning algorithms fall into three categories: supervised learning, unsupervised learning, and reinforcement learning

\subsubsection{Supervised Learning}

Supervised learning is the task of learning a function that maps the input features to desired output values [15]. Supervised learning algorithms receive a labeled dataset, where each sample in the dataset has a corresponding label or ground truth. Supervised learning can be further divided into classification and regression: 
- Classification: Classification models approximate a mapping from the input variables to a discrete output variable [16]. A classification model classifies the inputs into one of two or more classes. The performance of the model is often measured by the classification accuracy.

- Regression: Regression models approximate a mapping from the input variables to a continuous output variable [17]. The performance of a regression model is measured in terms of errors made in the model's predictions.

\subsubsection{Unsupervised Learning}

Unsupervised learning algorithms receive a set of input variables with no output variable or label [18]. The objective of unsupervised learning is to learn the underlying structure of the data and find an efficient representation for it. Two common unsupervised learning tasks are clustering and dimensionality reduction:

- Clustering: Clustering groups samples into clusters based on their similarities [19]. Samples which are similar to each other are grouped into the same cluster.

- Dimensionality Reduction: The idea of dimensionality reduction is to project samples from a high-dimensional space onto a lower dimensional space without losing much information [20]. This reduces the complexity of the data while retaining its structure.

\subsubsection{Reinforcement Learning}

Reinforcement learning is a class of machine learning where an agent interacts with the environment [21]. The goal of reinforcement learning is to train the agent in such a way that for a given environmental state, it chooses the optimum action that yields the highest reward.

\subsection{Overview of Throughput Prediction}

A widely known problem in wireless networks is throughput prediction, as it can be useful in many applications, such as adaptive bitrate (ABR) streaming [22] and resource allocation. Two common techniques that have been proposed for performing throughput prediction are connectivity maps and online throughput prediction described below. 


\subsubsection{Connectivity Maps}

The concept of connectivity maps is based on network coverage maps, which indicate the strength of the network coverage at different areas on the map. Similarly, connectivity maps indicate areas of high network quality and areas of low network quality. To construct a connectivity map, we need to collect information about various network quality parameters, such as RSSI, RSRP, RSRQ, SNR, and throughput, across the different areas on the map. One way to do that is to use vehicles as probes that measure the network quality [23]. Connectivity maps have been widely used for throughput prediction in the literature, as we will detail further in Section 2.5.

\subsubsection{Online Throughput Estimation}

Another technique for throughput prediction is the online throughput estimation, which estimates the instantaneous throughput based on the most recent measurements of the network quality metrics. This is often achieved using machine learning algorithms, such as linear regression and random forests, which take as input a set of network quality parameters along the corresponding throughput values and predict the future throughput. We further discuss how this technique has been used in the related work in Section 2.5.

\subsection{Related Work}

Over the past decades advancements in cellular networks required accelerating research findings in several fields. In this section, we focus on two major areas in the field of cellular networks: data collection and throughput prediction. An overview of the related work in these areas is provided in the following subsections.

\subsubsection{Data Collection}

To analyze the problem of changing wireless network connectivity levels in mobile settings, several data collection campaigns have been reported in the literature. In [1], Xu et al. proposed a system interface called PROTEUS that measures various network performance metrics, such as throughput, loss rate, and one-way delay of the network. Their approach was proposed for $3 \mathrm{G}$ networks but was not tested on $4 \mathrm{G}$ LTE 
networks. Abou-zeid et al. [2] also investigated wireless connectivity levels in $3 \mathrm{G}$ networks by conducting a network survey along the same bus route in Kingston, Ontario that we used. They performed 33 repeated bus routes, measuring the signal strength along with the GPS coordinates including a timestamp. They analyzed how the geographical, spatial and environmental conditions such as traffic congestion affected the signal strength variations. Another network survey was conducted on 3G networks by Margolies et al. in [3]. Using Samsung Galaxy S II Skyrocket phones as their measuring devices along with the QXDM toolset, they performed the measurements during different car trips, along highways and suburban roads, and also performed stationary measurements for the purpose of control. Furthermore, they investigated the influence of slow fading on the observed channel quality of $3 \mathrm{G}$ networks, which is experienced by vehicles moving between different cell towers.

In [24], Lu et al. conducted measurements in HSPA+ networks using the QXDM toolset. The measurements were conducted by connecting a mobile phone to a host laptop that runs the QXDM software and sending UDP packets to a mobile phone. The Nexus 5 phones were their measuring probes, and they performed 24 experiments with different mobility patterns including stationary, walking and driving. Yao et al. [25] conducted a network survey for eight months, performing 71 repeated car trips along a $23 \mathrm{~km}$ route in Sydney, Australia, that consisted of different radio conditions, such as terrestrial and underwater tunnels. Moreover, they relied on two different network operators for their measurements using HSDPA technology.

In [26], Han et al. carried out a measurement study with 38 repeated car trips along a $5 \mathrm{~km}$ route in the campus of Seoul National University, Seoul, South Korea. They measured the downlink throughput of video streaming from $3 \mathrm{G}$ and $4 \mathrm{G}$ LTE networks. They considered the variations in location, time, humidity, and speed. Through their study, they concluded that $3 \mathrm{G}$ networks are mostly affected by humidity and location, while 4G LTE networks are affected by speed, time, and location.

Jin in [7] investigated 4G LTE by conducting an extensive measurement study in the US with seven different mobile phones under various scenarios, different times of the day, different locations and different 
mobility patterns, including stationary, walking, local city driving and highway driving. The author collected a wide set of network parameters using the QXDM toolset.

Samba et al. in [27] used a crowdsourcing approach to collect network parameter measurements. Their network survey was conducted by 60 different users in France, who collected a total of 5700 measurements. Their measurements included the RSSP, RSRQ, throughput, distance to cell and speed, but they did not include the GPS locations of the users. To measure the throughput, the users downloaded a $32 \mathrm{MB}$ file.

In [23], Jomrich et al. conducted a network survey over three weeks, collecting over 74,000 throughput estimates along with other network quality parameters, such as RSRP, RSRQ, CQI, and SNR. For performing the measurements, they developed their own android application, and used multiple mobile phones. To measure the throughput, they sent and received a packet train of $750 \mathrm{~KB}$ of data to a dedicated server.

Lastly, Raca et al. [28] constructed a 4G LTE dataset consisting of various client-side network performance metrics. The data was collected from two Irish mobile operators, using different mobility patterns: stationary, walking, driving, riding in a bus and riding a train. They used the G-NetTrack Pro application on an android phone for conducting the measurements. To measure the throughput, they continuously downloaded and uploaded a $50 \mathrm{MB}$ file.

\subsubsection{Throughput Prediction}

Over the years, several researchers have investigated the problem of throughput prediction. Kamakaris and Nickerson have proposed the concept of using a connectivity map for throughput estimation in [29]. They investigated the relationship between the signal strength and the throughput in Wi-Fi networks. The authors found that the dynamic variations in the network conditions led to a short average lifetime of the connectivity map predictions. In [30], Pögel and Wolf also proposed the concept of using a connectivity map for predicting different network performance metrics, such as the RSSI, bandwidth and latency in a vehicular context. They also performed several drive tests to collect measurements of network performance parameters in an HSDPA network. Furthermore, in their later work [31], they used the data and information 
they gathered previously to enhance different network services such as adaptive video streaming and the handover between different network technologies.

In [1], Xu et al. used the system interface they developed, PROTEUS, for instantaneous throughput prediction. PROTEUS uses the previous 20 seconds of observed network performance and relies on regression trees for prediction. Liu et al. applied and compared seven different algorithms for mobile networks throughput prediction [32]. They used trace-driven data from 3G/HSPA networks to train their models. The measurements were collected in a stationary scenario and the model used the throughput during each 300 seconds to predict the throughput for the next 300 seconds.

In [7], Jin investigated the problem of applying throughput prediction algorithms in 4G LTE networks. They used the collected data using the QXDM toolset and developed LinkForcast, which is a machine learning based framework for throughput prediction. The framework uses lower-layer information to predict instantaneous link bandwidth. Samba et al. also performed throughput prediction in [27], using the random forest algorithm. For throughput prediction, they relied on additional data from network operators along with the data that they collected using a crowdsourcing approach. The additional data contained radio access network measurements such as the average cell throughput, average number of users in each cell and the connection success rate. The authors concluded that these additional measurements improved the throughput prediction accuracy.

\subsection{Summary}

In this chapter, we presented background information regarding the work discussed in this thesis. We explained the main concepts behind the work performed in the thesis and provided an overview about the work done in the literature in the fields of data collection and throughput prediction. In the next chapter, we explain our methodology for data collection and perform an analysis of the data we collected. 


\section{Chapter 3}

\section{Data Collection and Exploration}

In this chapter, the procedure of data collection, visualization and exploration is presented. In Section 3.1, we discuss our goals for data collection. In Section 3.2, we explain the various factors that influenced the data collection procedure. Section 3.3 presents the measurement setup and Section 3.4 shows the description of the dataset. In Section 3.5, we discuss the limitations of our procedure. Lastly, in Section 3.6, we present the data visualization and exploration results.

\subsection{Data Collection Goals}

The following are our goals for data collection:

1. Construct a comprehensive 4G LTE dataset of wireless network parameters, downlink, and uplink throughput and GPS location along a public transit bus route.

2. Analyze the cellular connectivity levels on public transportation in Kingston, Ontario.

3. Analyze the spatiotemporal correlation between signal strength and throughput.

4. Investigate the possibility of applying throughput prediction algorithms for anticipating locations and times with high or low network throughput.

To achieve these goals, we conducted a network survey using public city buses.

\subsection{Data Collection Factors}

Before starting our network survey, there were several important factors that had to be taken into consideration:

\section{1- Network Parameters}

For a thorough analysis of the network data and accurate throughput prediction, the dataset should include different wireless network's performance metrics such as RSRP, RSRQ, RSSI, SNR, downlink and uplink throughput and some context information such as location and speed the bus was travelling. 


\section{2- Granularity}

Granularity in network data collection refers to the intervals between different recorded measurements. A high granularity means a small interval between data records, and a low granularity means a long interval between data records. The measurement granularity is crucial for determining the accuracy of the throughput prediction algorithms; the higher the granularity, the more accurate the throughput prediction could be. Therefore, the data collection tool is required to have as high granularity as possible.

\section{3- Trip Times}

To account for discrepancies in the flow of road traffic as well as in the cellular network connectivity levels, we should perform the measurements at different times of the day. Ideally, they should include peak traffic hours as well as light traffic hours.

\section{4-Bus Route}

To be able to generalize our results on different areas across Kingston, the bus route should cover the major areas of the city, passing through urban and suburban areas.

\section{5- Cost}

The cost of the data collection process, equipment, and software required is an important factor to consider, especially for limited budgets.

\subsection{Measurement Setup}

To make our results comparable to other researchers' work, we chose to use an Android phone as our measuring tool. Principally, we used the G-NetTrack Pro Android application for obtaining the measurements, since it is capable of measuring different network quality parameters, downlink, and uplink throughput, as well as context-related information. The application has a one-second granularity; it logs measurements every second. Further advantages of using this application are its simple design and low cost. For throughput measurement, we continuously downloaded and uploaded a 10 MB file every two seconds, and recorded the achieved throughput using the G-NetTrack Pro application. A limitation of this application 
is that it uses transmission control protocol (TCP) for throughput measurement. As a result, the measured throughput is impacted by external factors such as congestion window, retransmission, and packet loss.

To investigate the applicability of throughput prediction on public transportation, we conducted the measurements along the Kingston Transit Express Bus 502 route (shown in Figure 3-1). The route has a length of $23.64 \mathrm{~km}$, with two major transfer points at Cataraqui Centre and Downtown Transfer Point. The route from the Downtown Transfer Point to Cataraqui Centre is urban, while the route along Bayridge Drive is suburban. We recorded the measurements by taking three bus trips every day (Monday to Friday) at: 9 am, $12 \mathrm{pm}$ and $6 \mathrm{pm}$. Each trip was the same route, had the same starting and ending points, and too under one hour to complete, starting and ending at the same times every day.

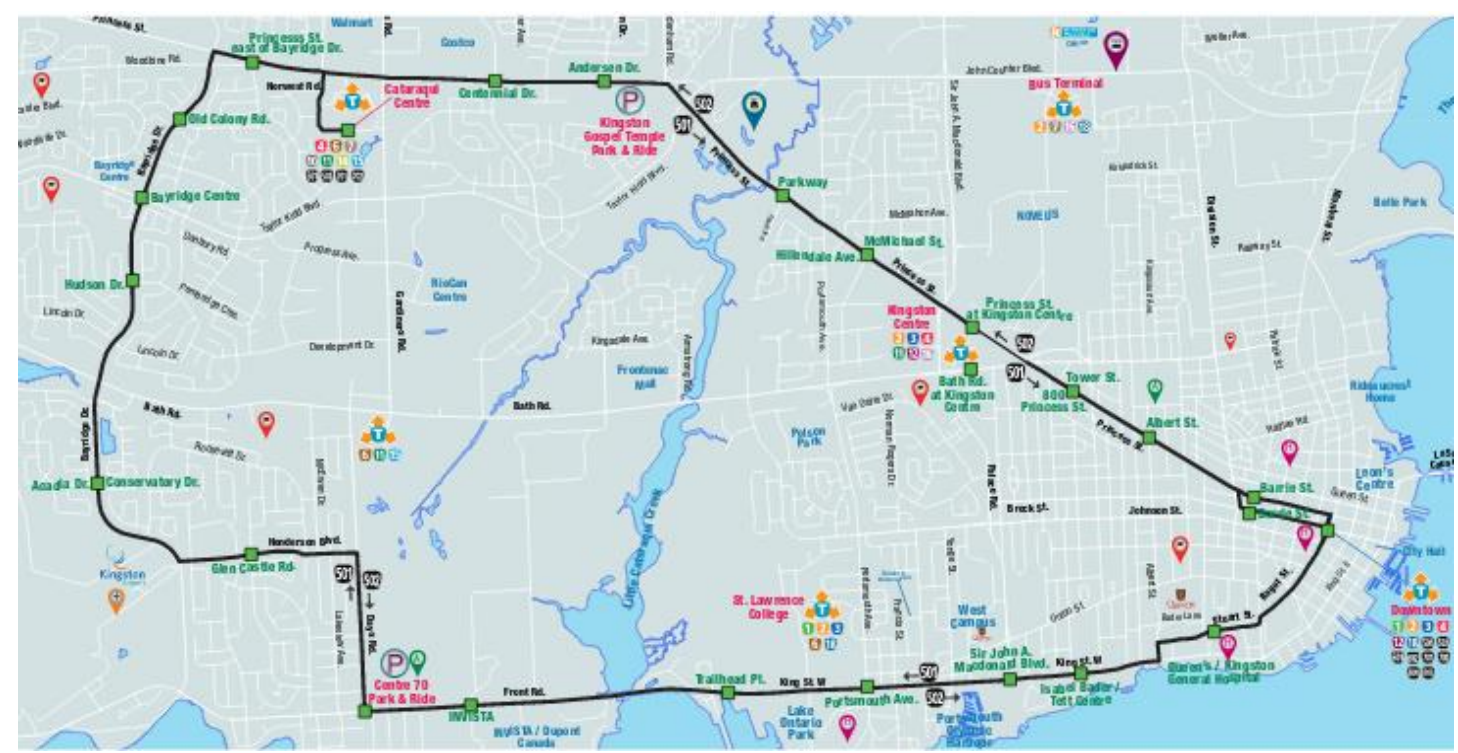

Figure 3-1: The 23.64 km trajectory of the Kingston Transit Express Bus 502.

Source: Reprinted from Kingston Transit, City of Kingston, retrieved from https://www.cityofkingston.ca.

\subsubsection{First Experiment}

We started our network suvey using a Samsung Galaxy S6 phone (LTE Category 6) on the 502 bus and collected data starting at $9 \mathrm{am}, 12 \mathrm{pm}$ and $6 \mathrm{pm}$. After analyzing the first data set, we faced a major problem. Although the phone was able to measure the different network quality parameters as well as the downlink 
and uplink throughput, it was not able to record the GPS locations of the entire trip; there were several missing GPS locations, as shown in Figure 3-2. This problem was due to the inaccurate GPS solution of the Samsung Galaxy S6. To solve this problem, we had to use another device with a more robust GPS solution.

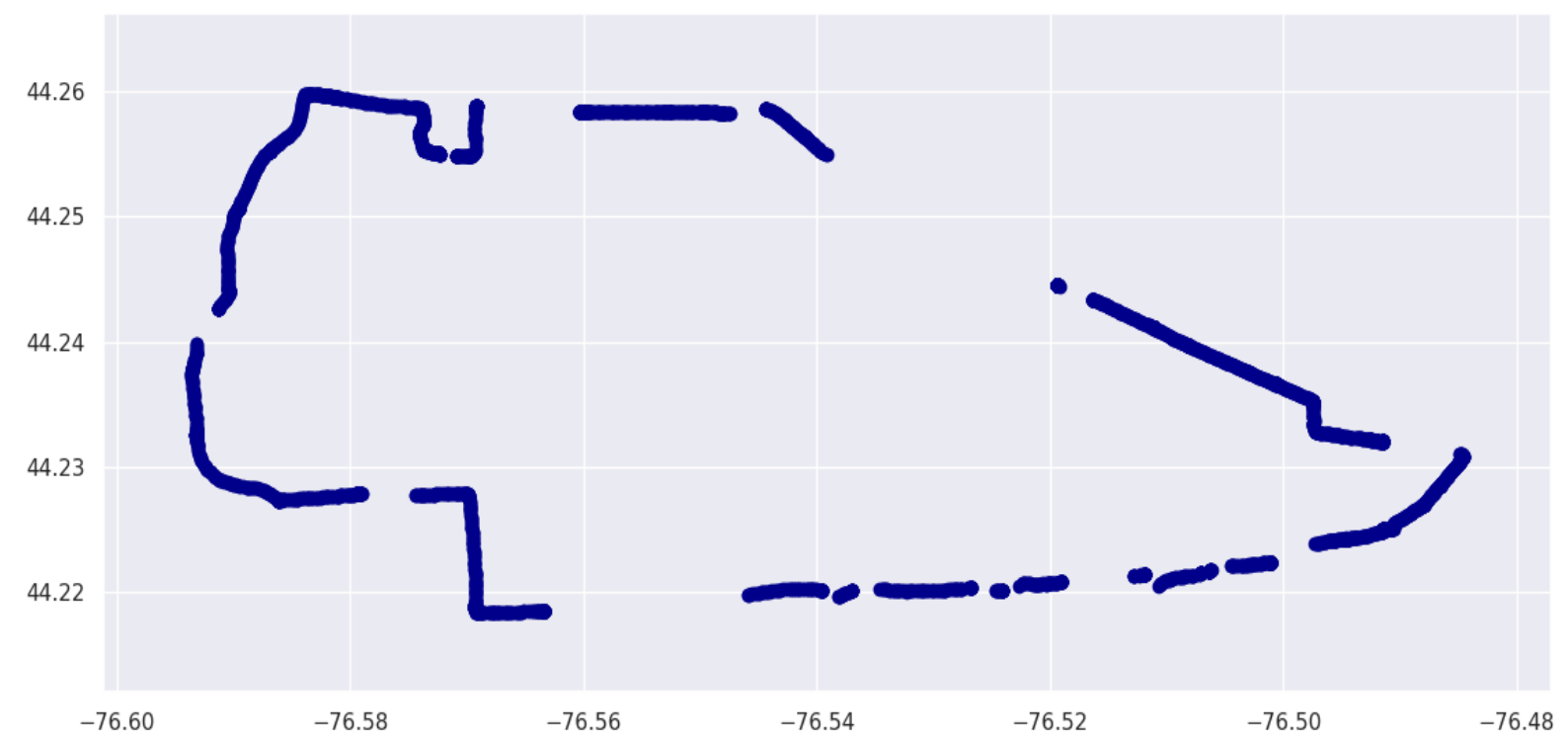

Figure 3-2: Sample trip trajectory showing incomplete GPS recordings.

\subsubsection{Second Experiment}

For the second experiment, to ensure our results are accurate and to compare different LTE categories, we decided to use two different android phones. We used a Samsung Galaxy S9 (LTE Category 18) and a Samsung Galaxy S10e (LTE category 20), which were both able to record the wireless network parameters that we needed and did not have an issue with the GPS solution. The new phones also achieved higher throughput readings than the Samsung Galaxy S6. For this experiment, we also collected data at 9 am, 12 $\mathrm{pm}$ and $6 \mathrm{pm}$. The analysis presented in this thesis is based on the data collected during the second experiment. 


\subsection{Dataset Description}

The following are the attributes of the dataset:

- Timestamp: timestamp of the measurement, precise time when the measurement is taken.

- Longitude: one of the GPS coordinates of the mobile device.

- Latitude: one of the GPS coordinates of the mobile device.

- Speed: speed of the bus at the time of measurement in $\mathrm{km} / \mathrm{h}$, calculated from the GPS data.

- Operator: the mobile country code (MCC) and mobile network code (MNC), which are used together to identify a mobile network operator uniquely.

- CellID: cell ID of serving cell.

- LAC: location area code of serving cell, a unique identifier used by each public land mobile network (PLMN) to update the location of mobile subscribers.

- NetworkTech: current technology, could be 2G, 3G, or 4G.

- RSSI: received signal strength indicator, a measure of the power present in a received radio signal [33].

- RSRP: reference signal received power; this is the measure of power of the LTE reference signals spread over the full bandwidth and narrowband.

- RSRQ: reference signal received quality, indicates the quality of the received reference signal.

- SNR: signal-to-noise ratio, which is the ratio of signal power to the noise power, expressed in decibels.

- Downlink bitrate: current downlink bitrate at the time of measurement expressed in kbps.

- Uplink bitrate: current uplink bitrate at the time of measurement expressed in kbps.

- Height: height of the measuring device over ground level.

- $\quad$ N-Cellid - ID of neighboring cell.

- N-RSRP: RSRP of neighboring cell.

- N-RSRQ: RSRQ of neighboring cell. 


\subsection{Limitations of our Approach}

Below are a few of the limitations that we encountered during our network survey:

\section{1- Data Plan}

As we mentioned earlier, for measuring the throughput, we had to keep downloading and uploading a 10 MB file every two seconds. This led to massive amounts of data being used, that by the end of our network survey, our data usage was flagged.

\section{2- Data from Network Operators}

For privacy reasons, network operators often refuse to make their data public. For this reason, we only had access to the operator's client-side data. Network operators can offer information about the cellular network performance, such as the average cell throughput, the average number of users, the connection success rate and the Block Error Ratio [34]. We believe that having access to such measurements could have improved the performance of throughput prediction algorithms.

\section{3- Budget}

Because of the limited budget and the data plan costs, we could not use more than two phones. Performing the measurements with more than two phones could improve the accuracy of the measurements and therefore lead to better prediction results.

\subsection{Data Exploration}

In this section, we explore the various attributes of the data, discuss their variation with respect to time and location, and analyze the correlation between the features.

\subsubsection{Signal Strength Variation}

Shown in Figure 3-3, Figure 3-4, and Figure 3-5 are the sample latitude and signal strength (RSRP) vs. time for the three trips. We display the latitude graphs on top of the signal strength graphs to show the signal strength with respect to both time and location, and to show the effect of bus delays. The variations between the trips could be caused by a variety of factors, like traffic lights, bus stops, road traffic levels, and the behavior of different drivers. From Figure 3-3 to Figure 3-5, we can see that the 6-pm-trip exhibits more 
time variations than the other two trips; the variance in the latitude is higher than in the 9-am-trip and the 12-pm-trip. This could be due to heavier road traffic or a higher level of passenger activity on the bus at 6 $\mathrm{pm}$, as this is the rush hour.

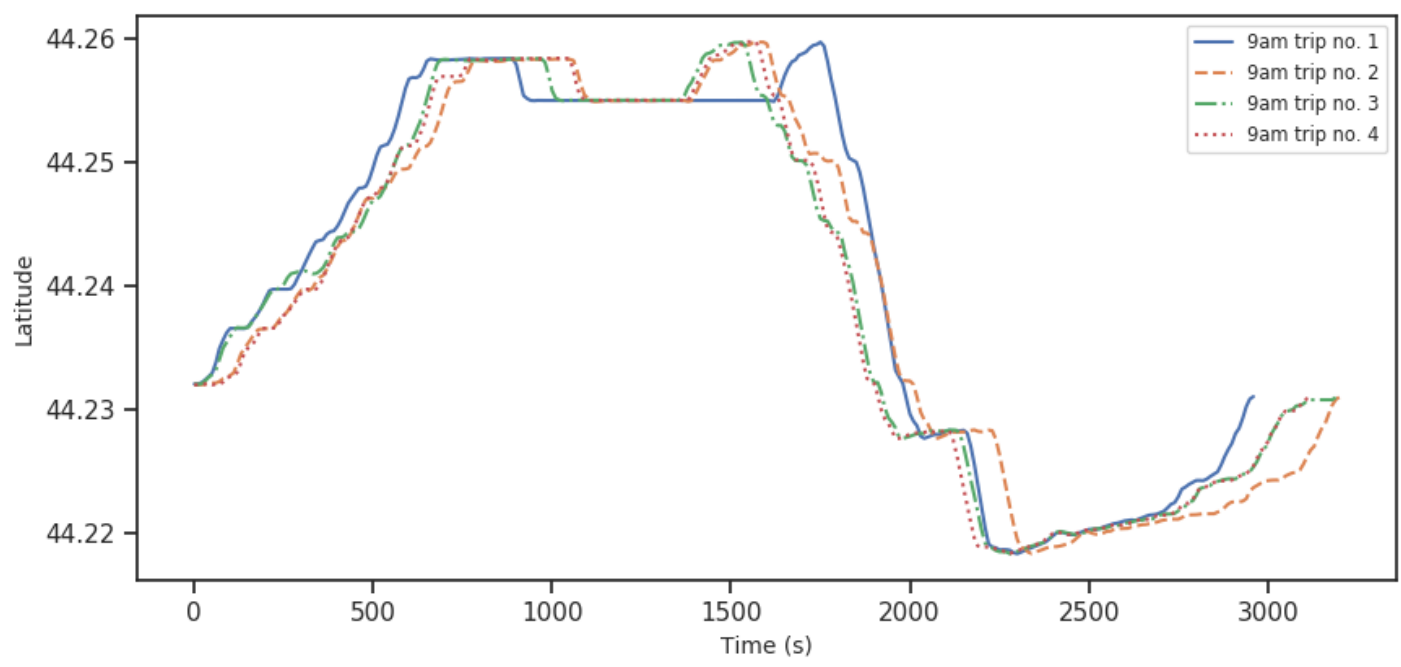

(a) Latitude vs. Time.

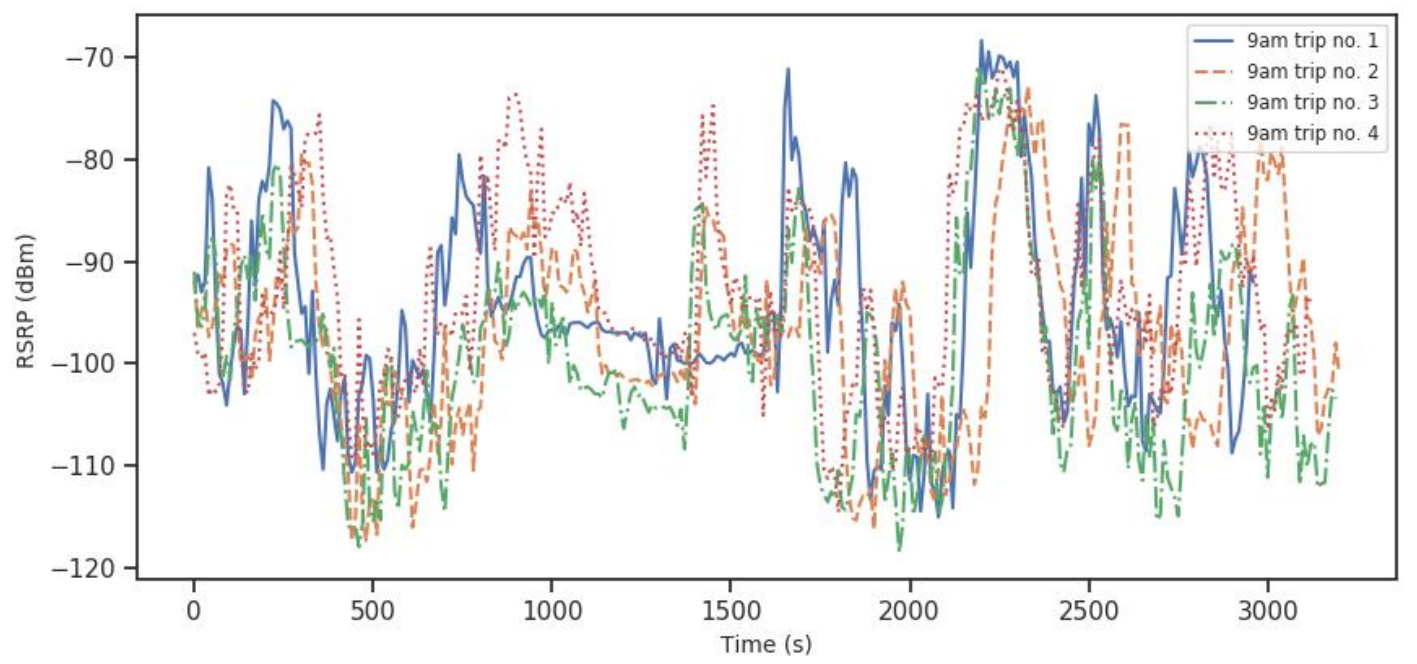

(b) RSRP vs. Time.

Figure 3-3: Sample 9-am-trips (a) latitude and (b) signal strength variation per second. 


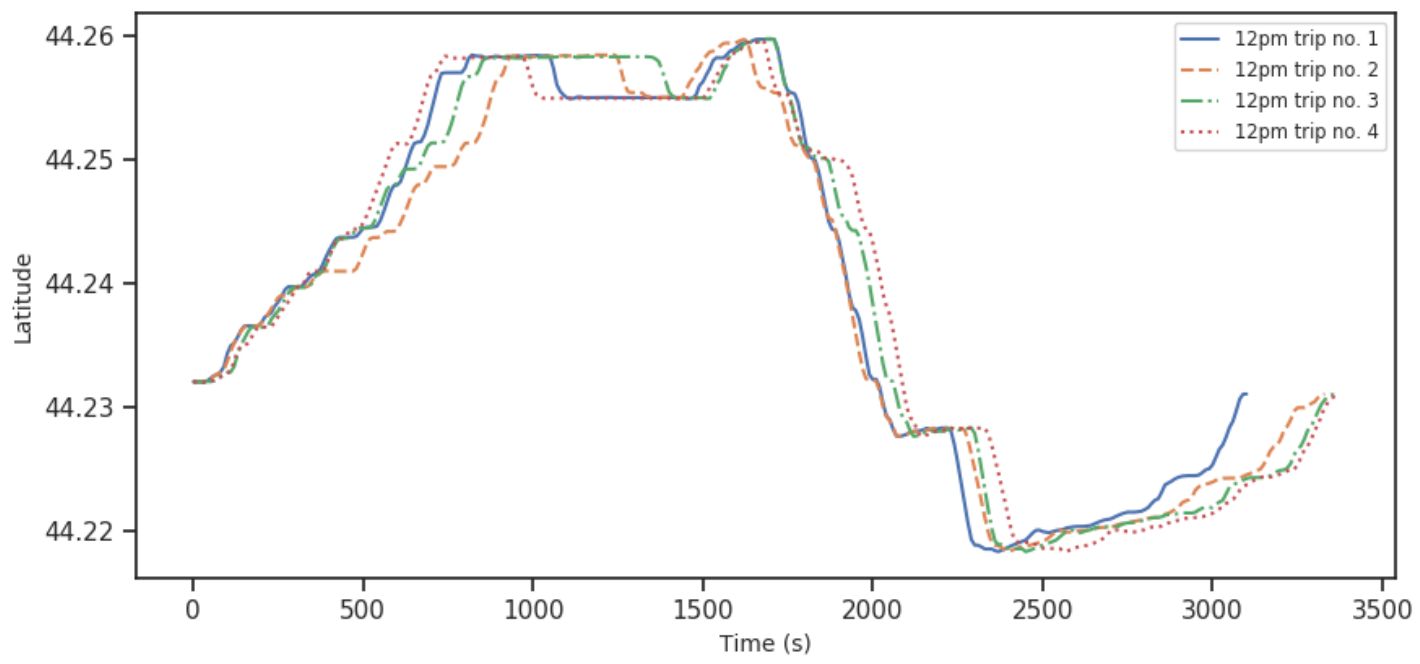

(a) Latitude vs. Time.

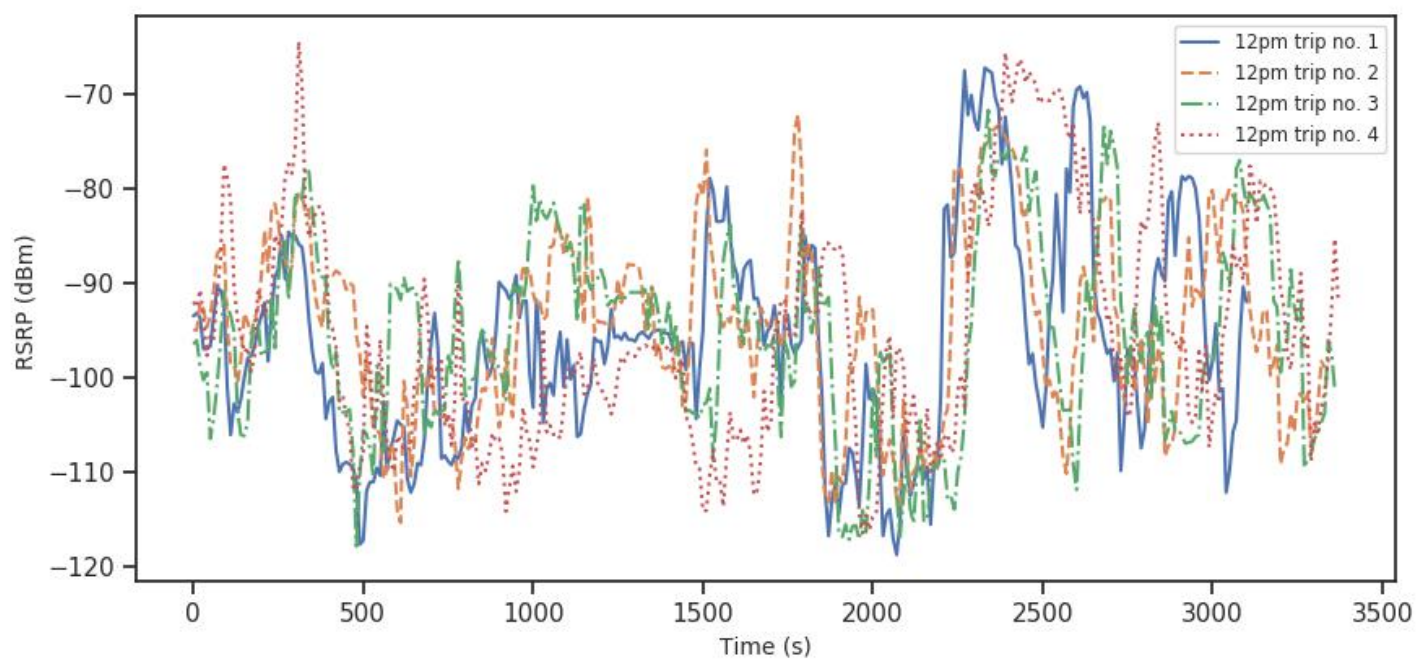

(b) RSRP vs. Time.

Figure 3-4: Sample 12-pm-trips (a) latitude and (b) signal strength variation per second. 


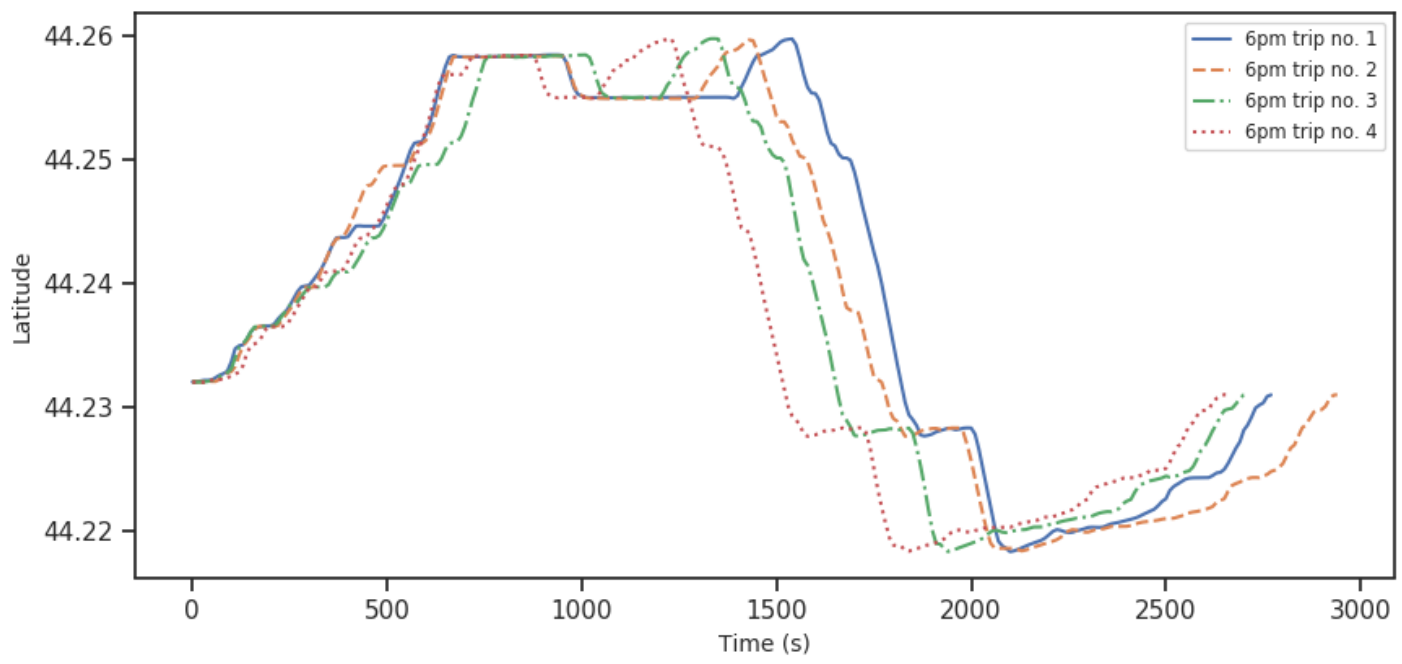

(a) Latitude vs. Time.

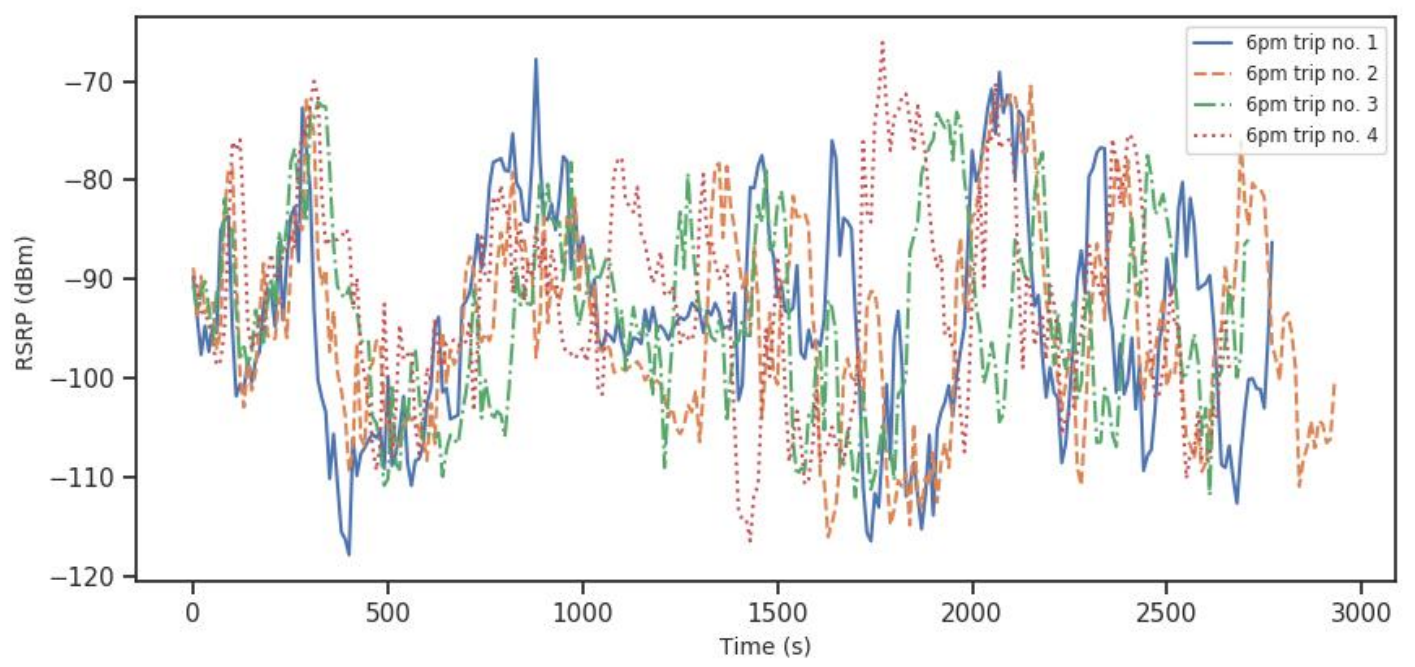

(b) RSRP vs. Time.

Figure 3-5: Sample 6-pm-trips (a) latitude and (b) signal strength variation per second.

It is important to note that these observations differ from those made by $\mathrm{H}$. Abou-zeid in [2], which indicated that the measurements were more consistent in the 6-pm-trip than in the 12-pm-trip, as at $6 \mathrm{pm}$ there was less road traffic. We believe that this change in conditions is due to the city's development in the last five years and the evolution of cellular networks from $3 \mathrm{G}$ to $4 \mathrm{G}$. 
Moreover, we can observe two main consistent dips in the signal strength graphs at 500s and 2000s. These correspond to GPS coordinates (44.24485680, -76.51957566) and (44.22804575, -76.58746690), which are located at Princess Street near Kingston Center and Bayridge Drive, respectively. We can see that the suburban area along Bayridge Drive suffers from a relatively long period of low signal.

From Figure 3-3 and Figure 3-4, one observes that there is not much variation in the latitudes as in Figure 3-5. The 9-am-trips and 12-pm-trips are much more consistent with respect to the location variance. However, some minor variations due to context changes i.e., road traffic conditions and number of passengers on the bus are normal.

Figure 3-6 shows a heat map of the average signal strength variation at different locations along the bus route. We can see there are areas of poor signal, such as on the road to Cataraqui Centre on Princess Street and on Bayridge Drive. On the other hand, areas with more demand such as Downtown, Cataraqui Centre, Invista Centre, St. Lawrence College, are shown to have a higher signal strength. This is consistent with the fact that there are cell towers located in each of these areas, as shown in the map in Figure 3-7, in order to keep up with the cellular network demand.

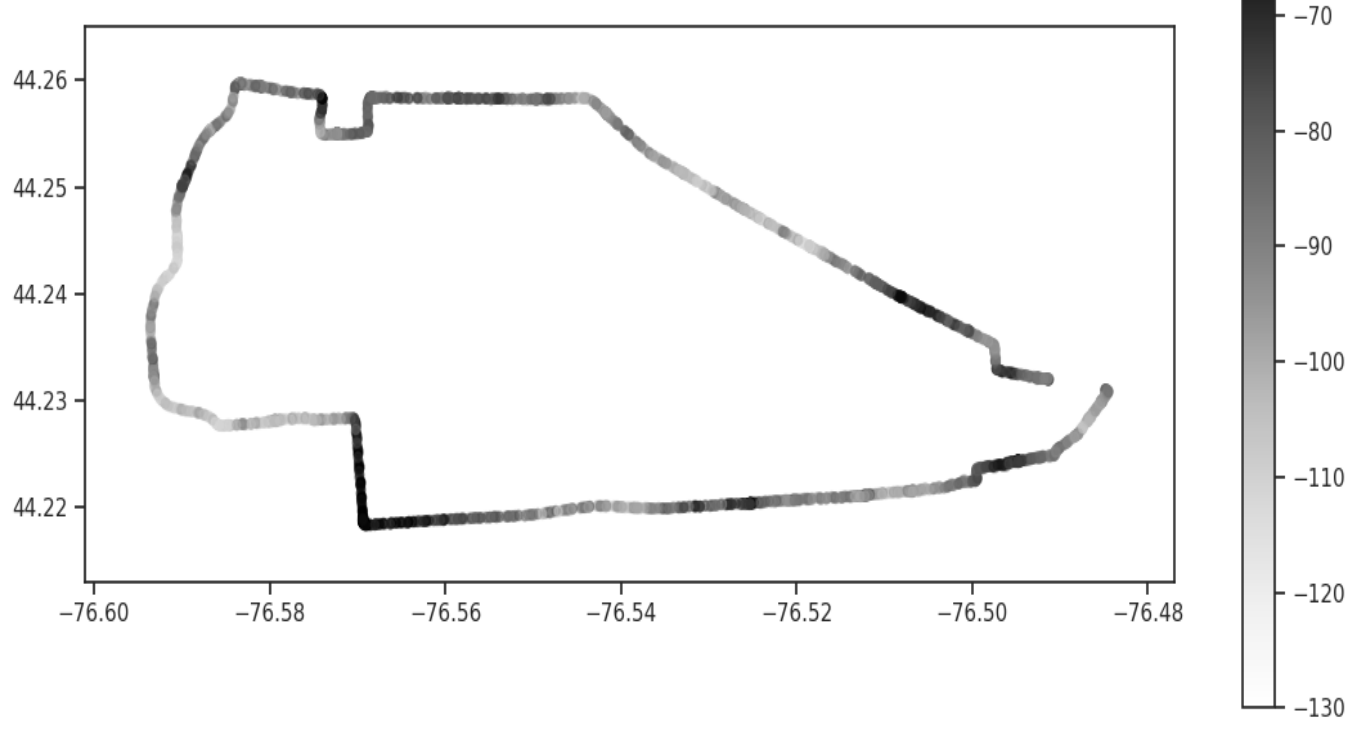

Figure 3-6: Average signal strength map. 


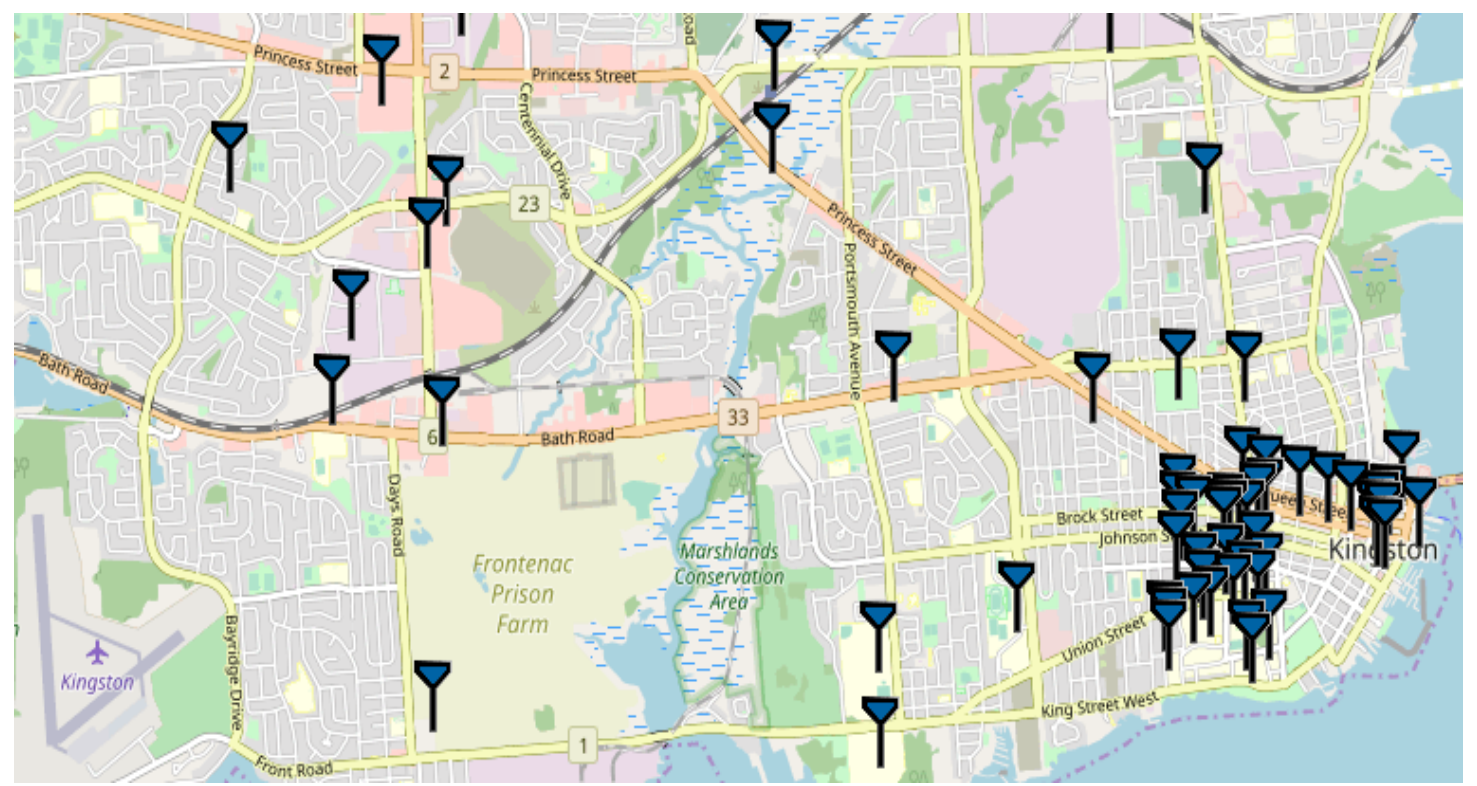

Figure 3-7: Operator's cell tower locations in Kingston.

Source: Reprinted from Canadian Cellular Towers Map, by Steven Nikkel, retrieved from https://www.ertyu.org/steven_nikkel/cancellsites.html/ Copyright 1997-2019 by Steven Nikkel.

The density plots in Figure 3-8 represent the signal strength distributions at each of the three different times. We can infer from these plots that at the 6-pm-trips have a lower signal strength mean. This is possibly caused by increased road traffic at that time; busier traffic hours lead to a heavier network traffic, which in turn reduces the signal strength. Conversely, the distributions for the 9-am-trips and 12-pm-trips have noticeably higher signal strength values. Additionally, we can see that the signal strength at the three different times of the day follows a Gaussian distribution [35], with a mean ranging from -93 to $-96 \mathrm{dBm}$ and a standard deviation around 10. 


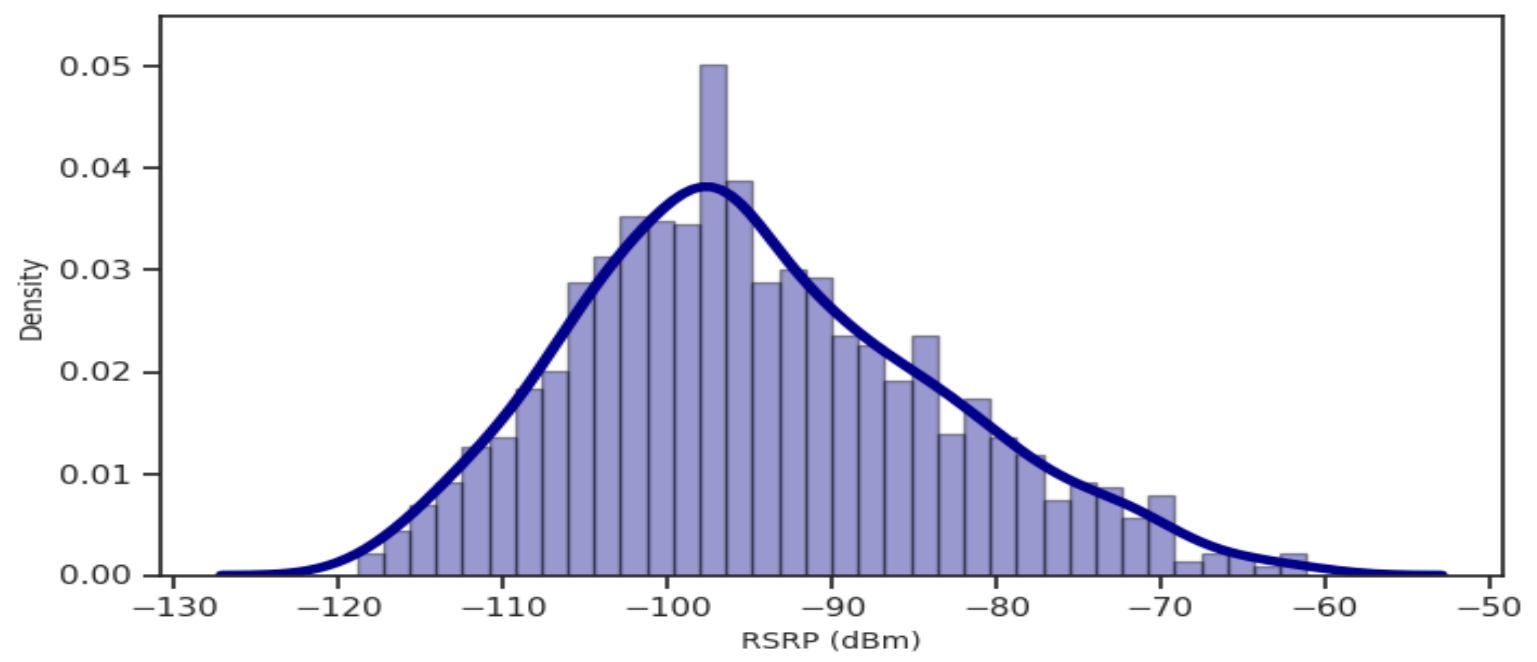

(a) RSRP density plot for the 9-am-trip.

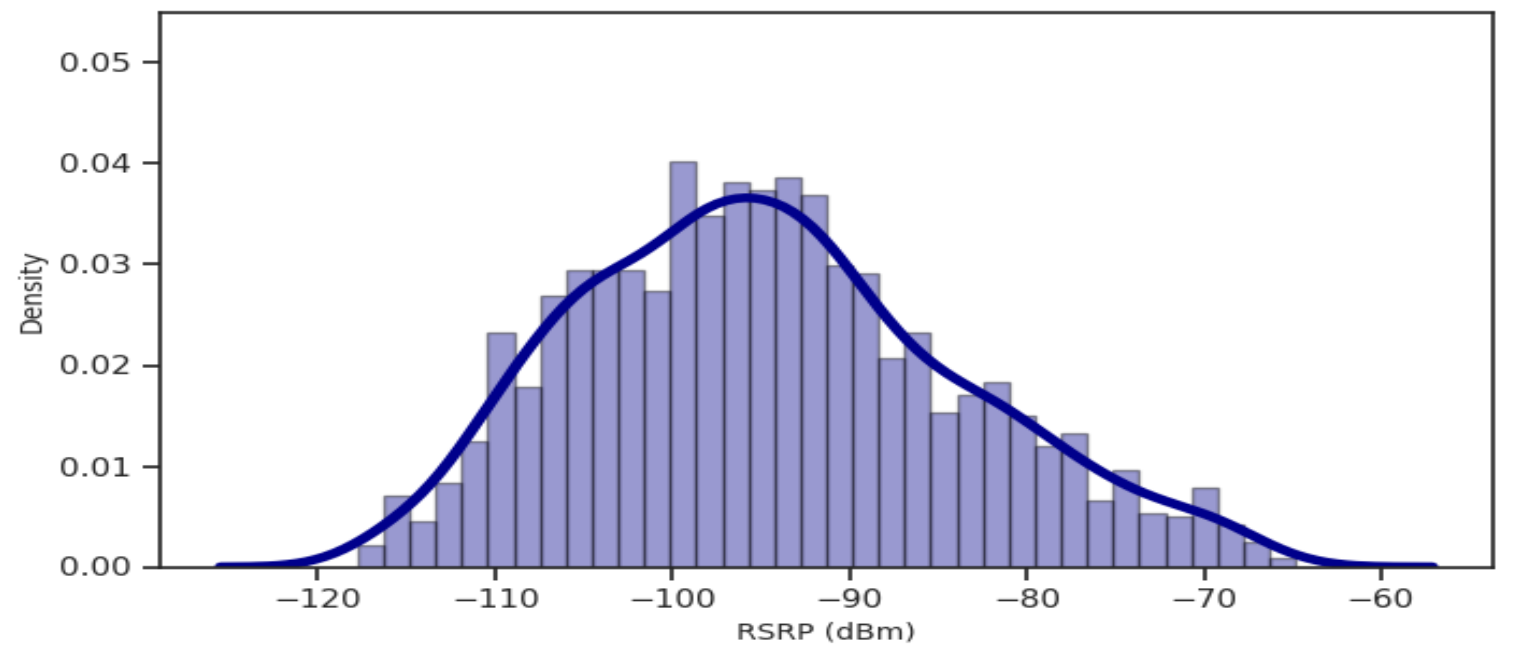

(b) RSRP density plot for the 12-pm-trip.

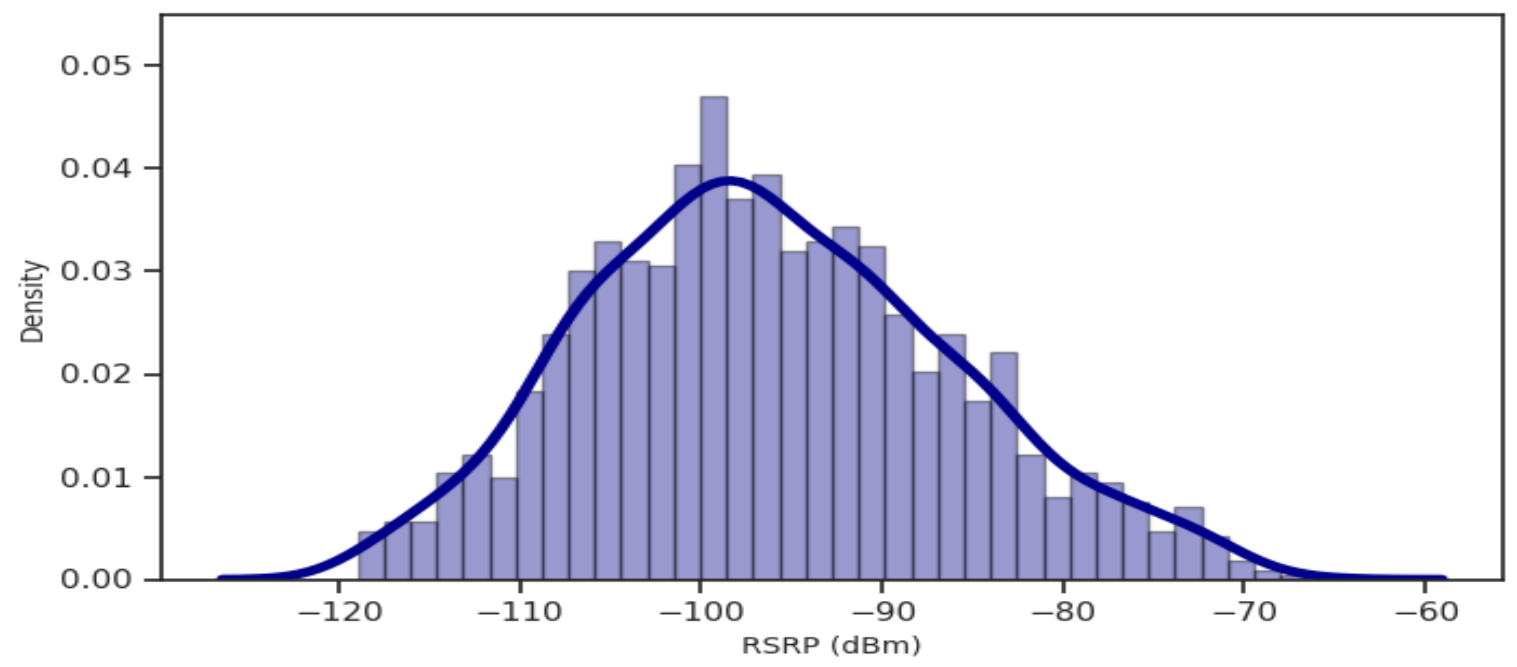

(c) RSRP density plot for the 6-pm-trip.

Figure 3-8: Density plots for signal strength at (a) $9 \mathrm{am}$, (b) $12 \mathrm{pm}$, and (c) 6pm. 
In addition to the density plots, we also generated variance maps for the three times of the day in order to analyze the geographical and temporal effects on the variance of the signal strength. The results are displayed in Figure 3-9, Figure 3-10, and Figure 3-11; in these figures darker shades along the route indicate regions of higher variance. One can see from the plots that signal strength variance per location is significantly higher at 9 am than at $12 \mathrm{pm}$ and $6 \mathrm{pm}$. Based on this observation, we can infer that the signal strength variance at different locations are more consistent at $12 \mathrm{pm}$ and $6 \mathrm{pm}$ than at $9 \mathrm{am}$. Moreover, one can see that the area around Cataraqui Centre has a higher signal strength variance at different times of the day than the rest of the route, as it is consistently high in the three figures.

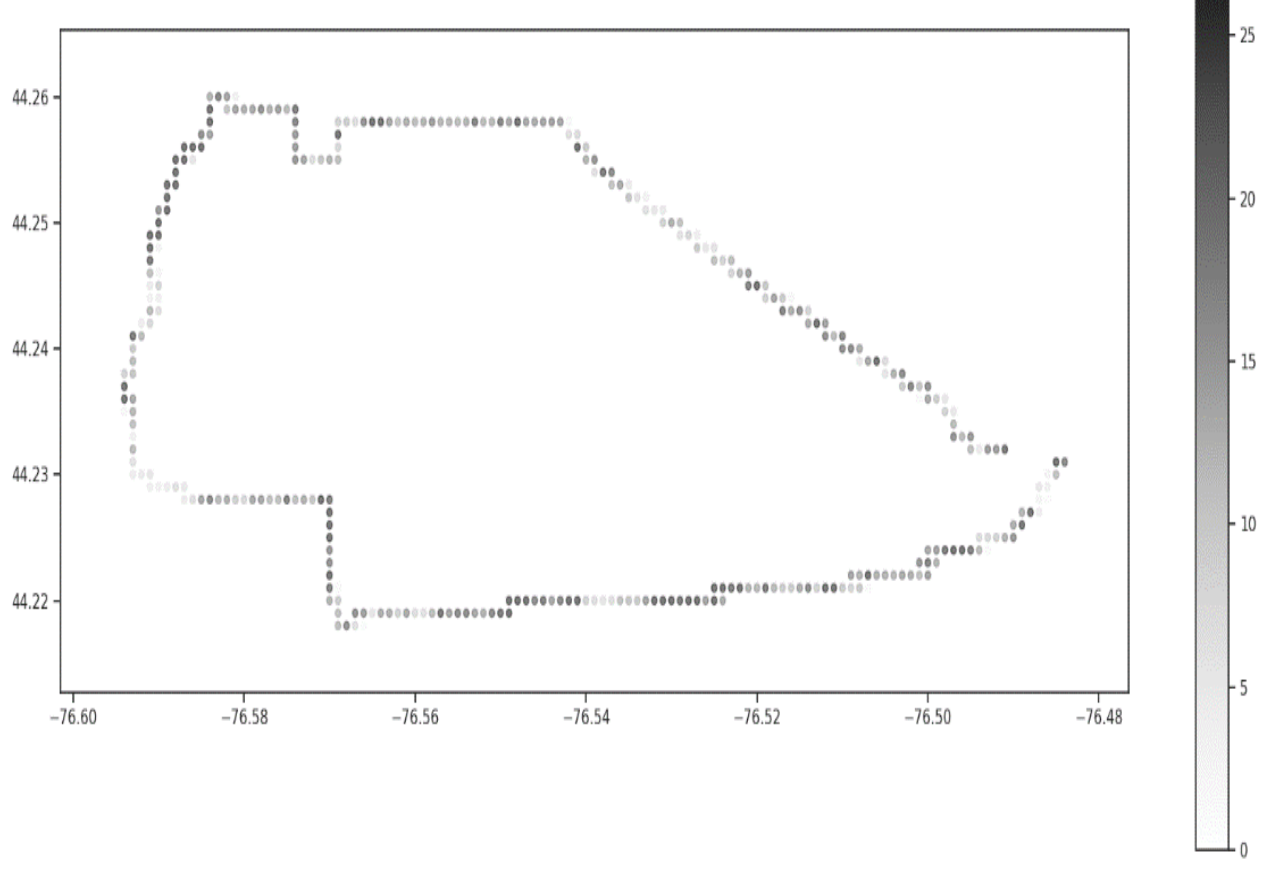

Figure 3-9: Variance of signal strength map for the 9-am-trip. 


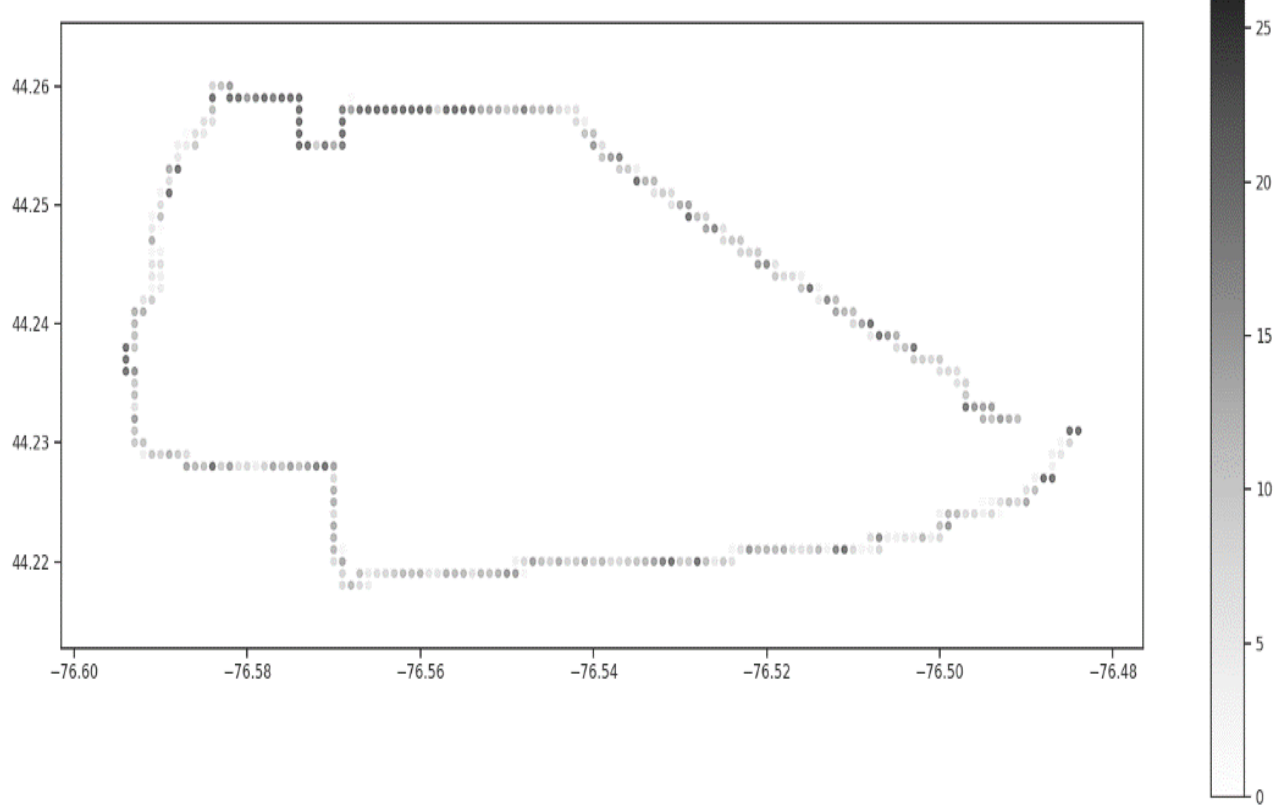

Figure 3-10: Variance of signal strength map for the 12-pm-trip.

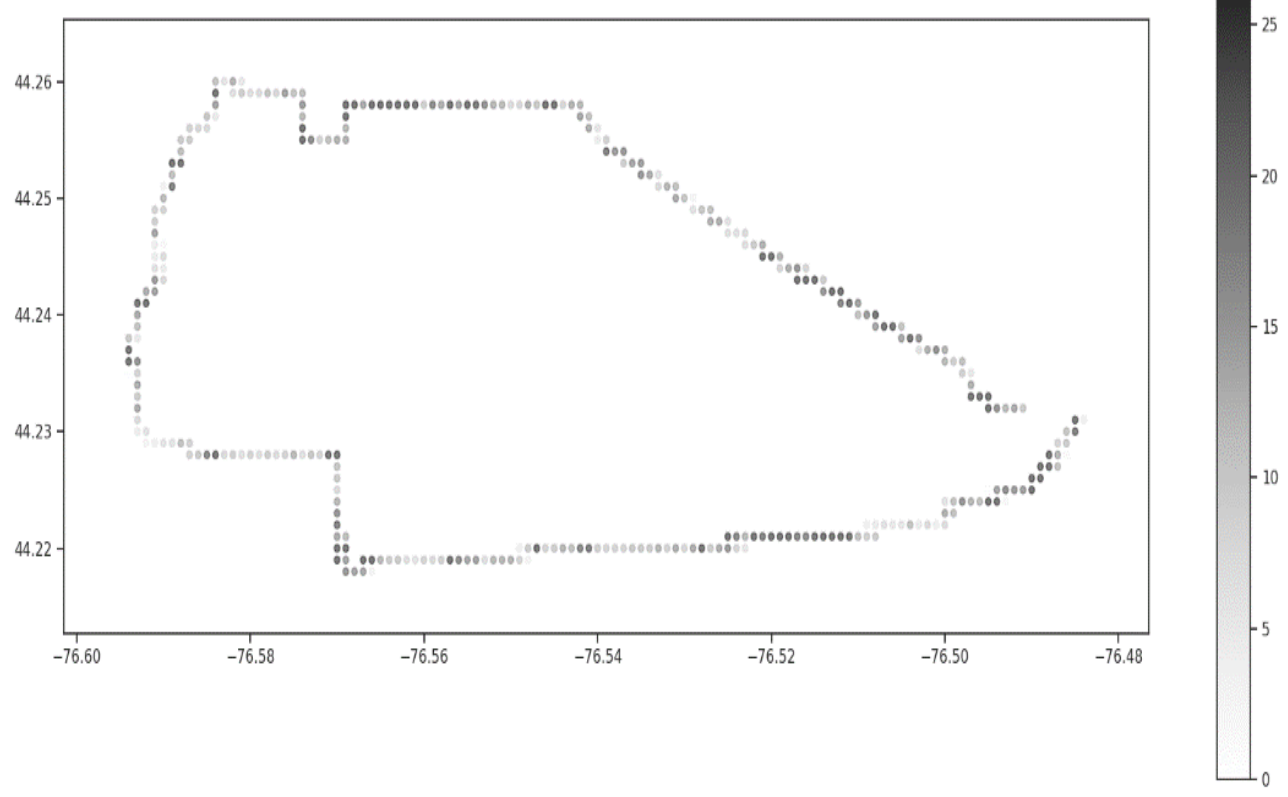

Figure 3-11: Variance of signal strength map for the 6-pm-trip. 


\subsubsection{Throughput Variation}

The following Figure 3-12, Figure 3-13, and Figure 3-14 display sample downlink throughput values at the 9-am-trips, 12-pm-trips and 6-pm-trips, respectively. In a similar manner to the signal strength, the throughput variation at the 6-pm-trips is much higher than at the other times of the day. In the three figures, we can observe some major dips in the throughput at 500s, 2000s, and 2900s, which correspond to GPS coordinates of $(44.24909641,-76.52827461),(44.22807929,-76.58608385)$, and $(44.22177393$, 76.50510436). These coordinates are located at Princess Street near Kingston Center, Bayridge Drive and King Street near Kingston General Hospital, respectively. This is consistent with the low signal strength measurements recorded in these areas, which are caused by the lack of cell towers in these locations.

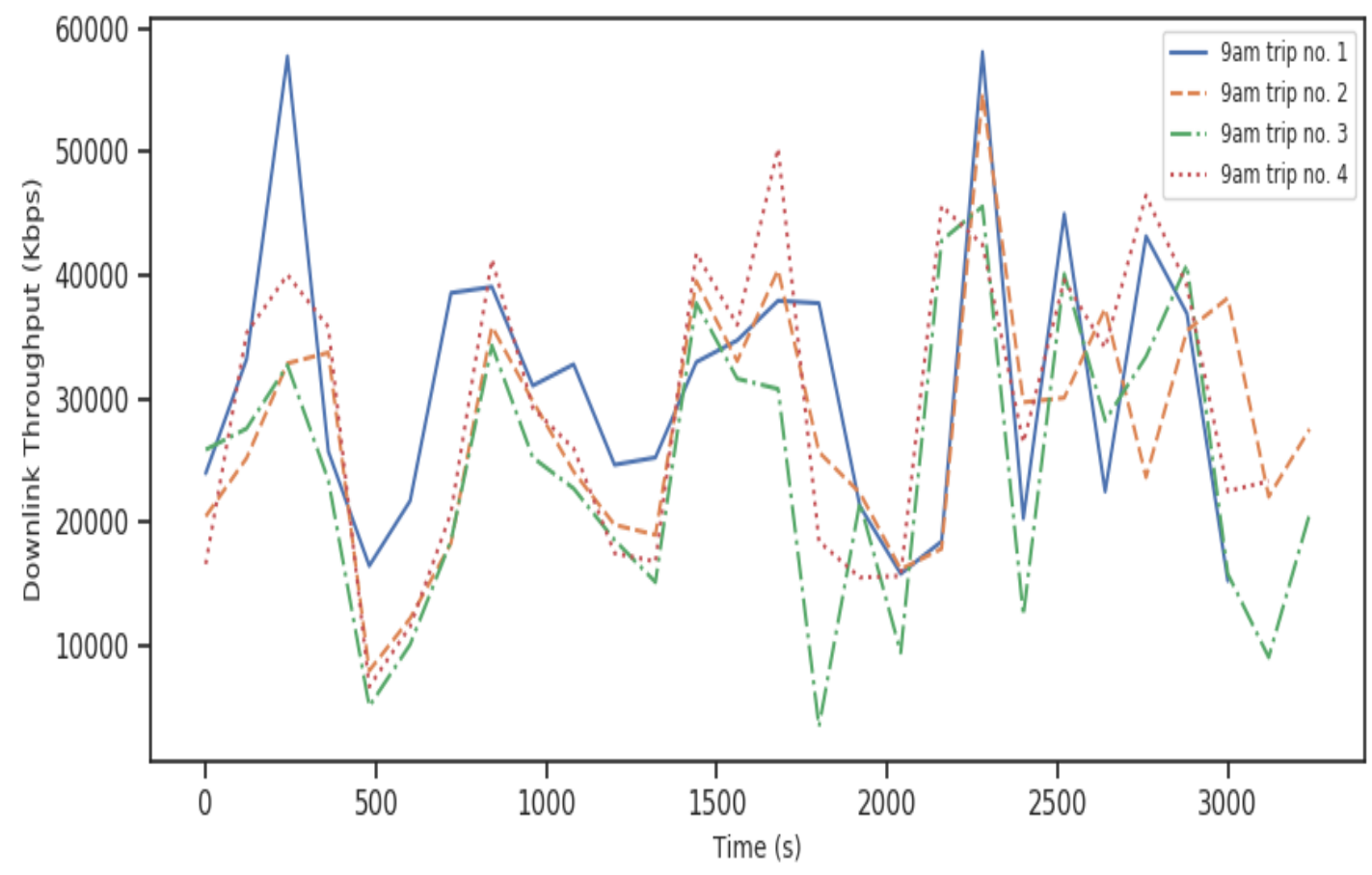

Figure 3-12: Throughput variation per second for sample bus 9-am trips. 


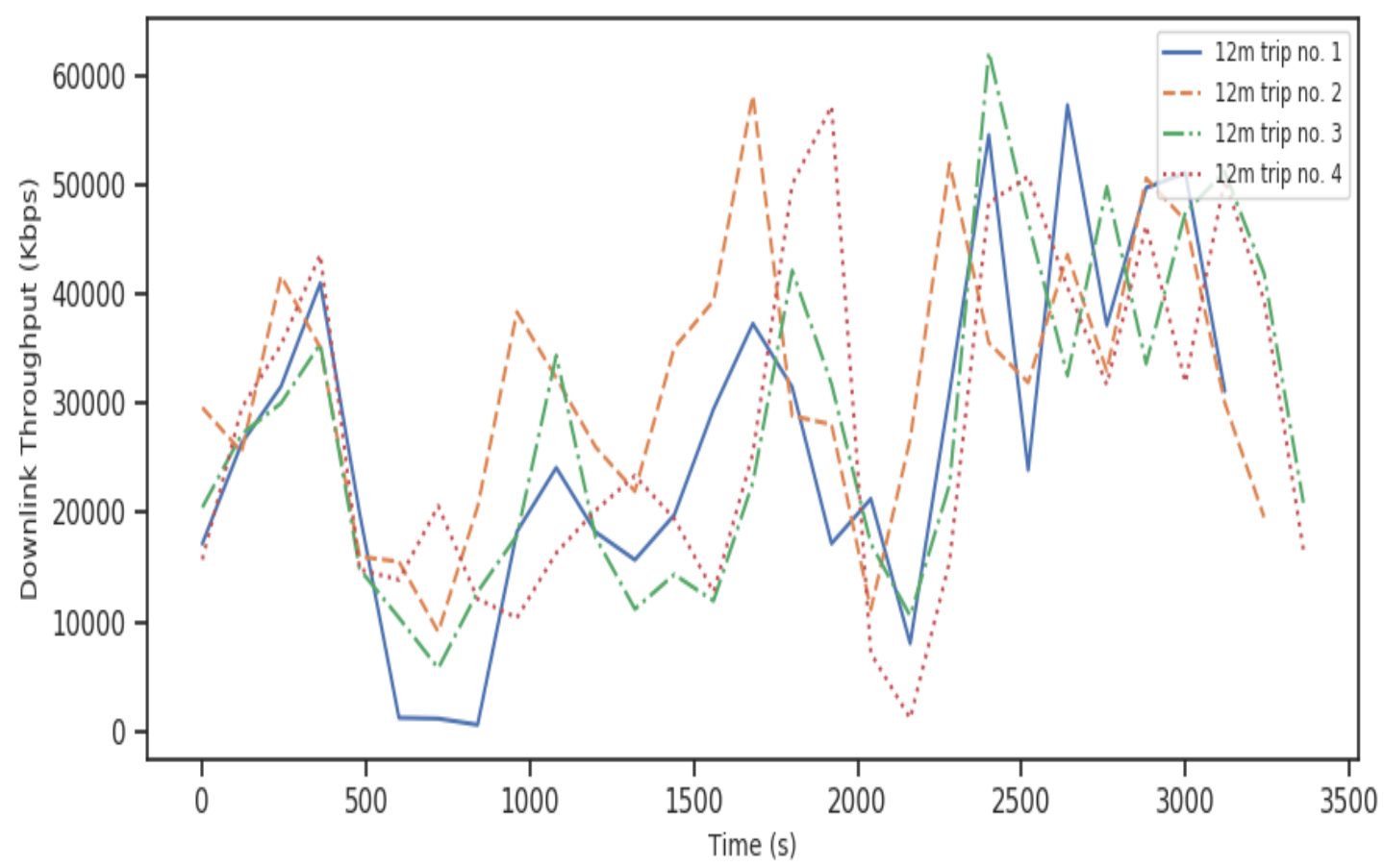

Figure 3-13: Throughput variation per second for sample 12-pm-trips.

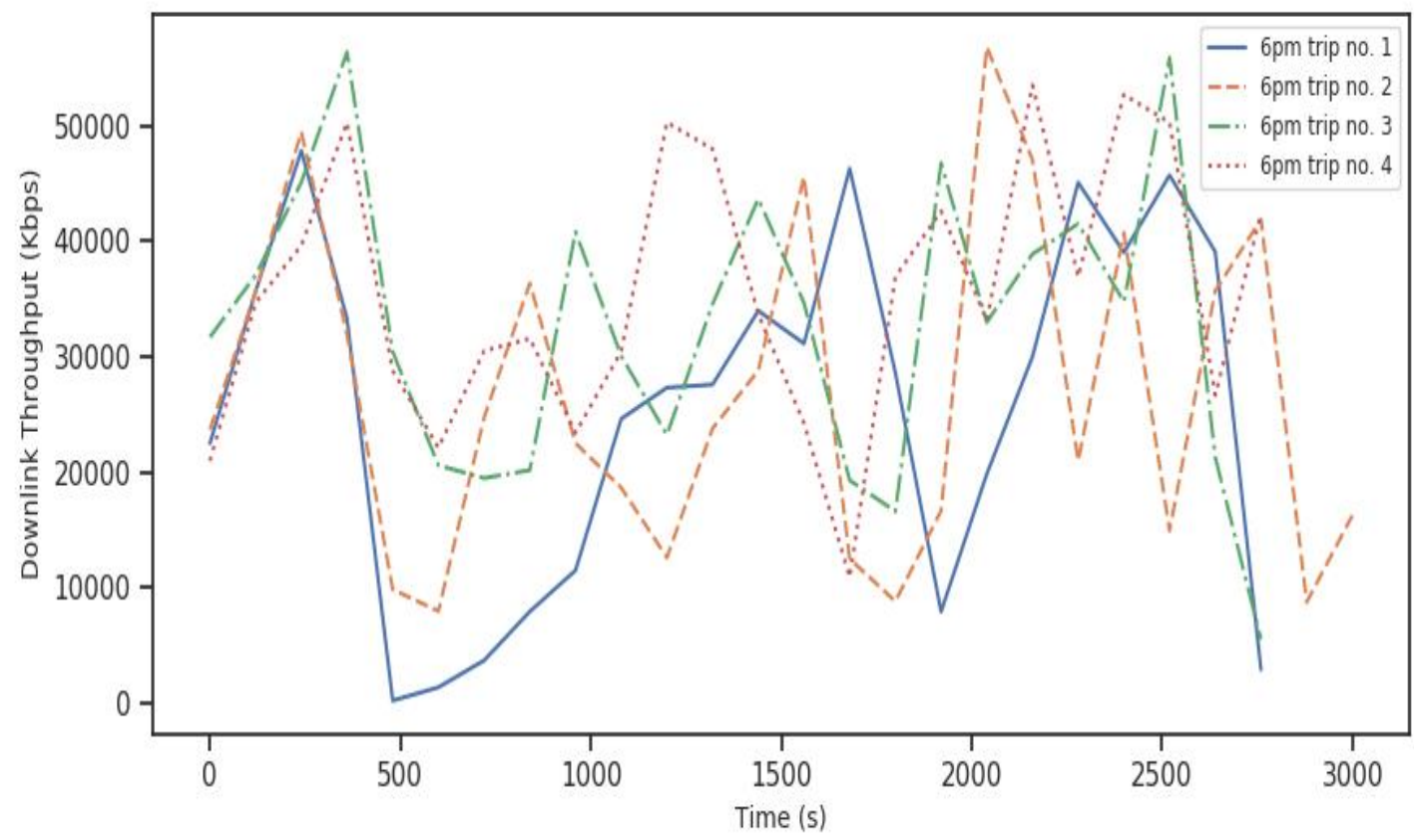

Figure 3-14: Throughput variation per second for sample 6-pm-trips. 
The heatmap in Figure 3-15 shows the variation of the average downlink throughput along the bus route. Consistently with the signal strength values in Figure 3-6, the achieved throughput is low on the way to Cataraqui Centre and on Bayridge Drive.

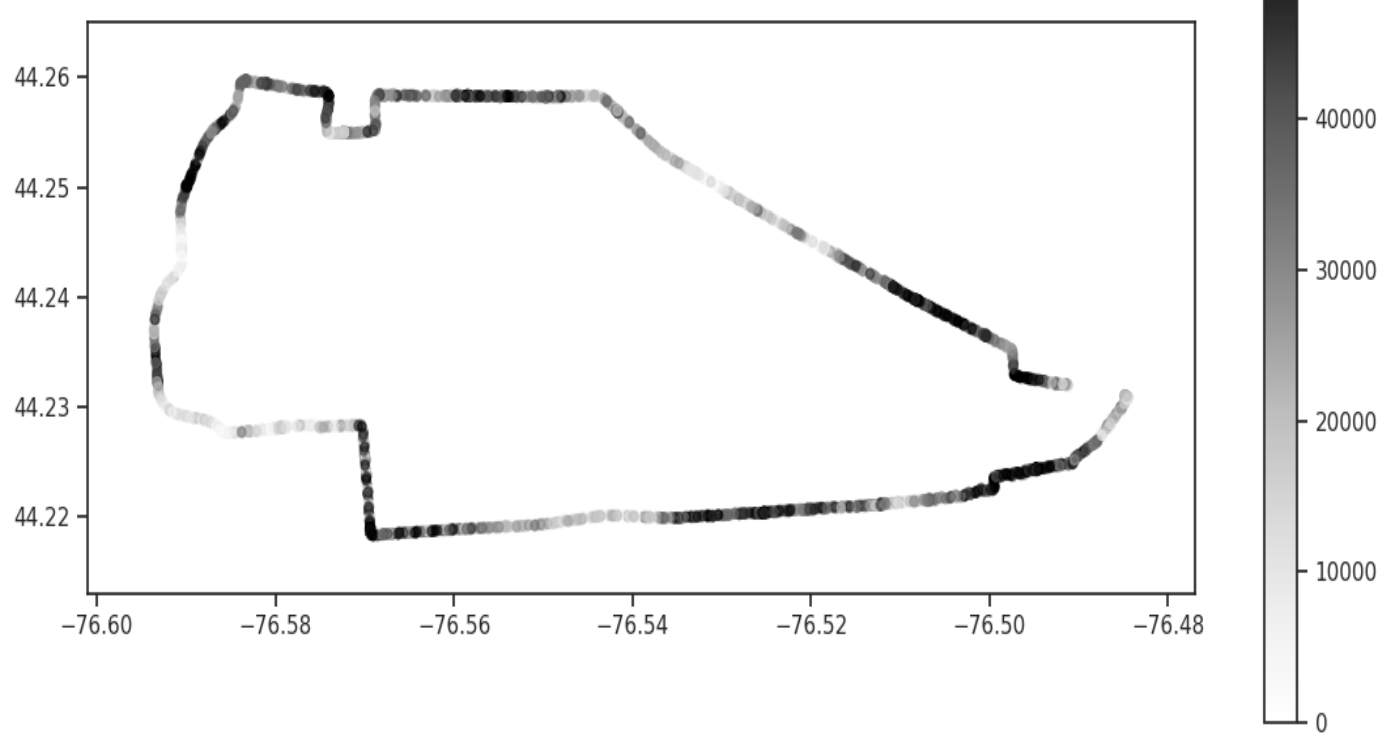

Figure 3-15: Average throughput map.

Figure 3-16 shows the density plots of the throughput, which indicate the throughput distributions at each of the three different times. We can note that the throughput measurements in the 9-am-trips have a Gaussian distribution, while those in the 12-pm-trips and 6-pm-trips have a bimodal distribution [36]. The bimodal distribution can be inferred from the two peaks in the plots, and this usually indicates that the data may be coming from two different distributions. Consistently with the signal strength, the throughput in the 6-pm-trips demonstrates lower values. On the other hand, the distributions for the 9-am-trips and 12-pmtrips exhibit higher throughput values. The mean throughput in the 6-pm-trips is $26 \mathrm{Mbps}$, while it is 28 Mbps and 30 Mbps in the 9-am-trips and 12-pm-trips, respectively. 


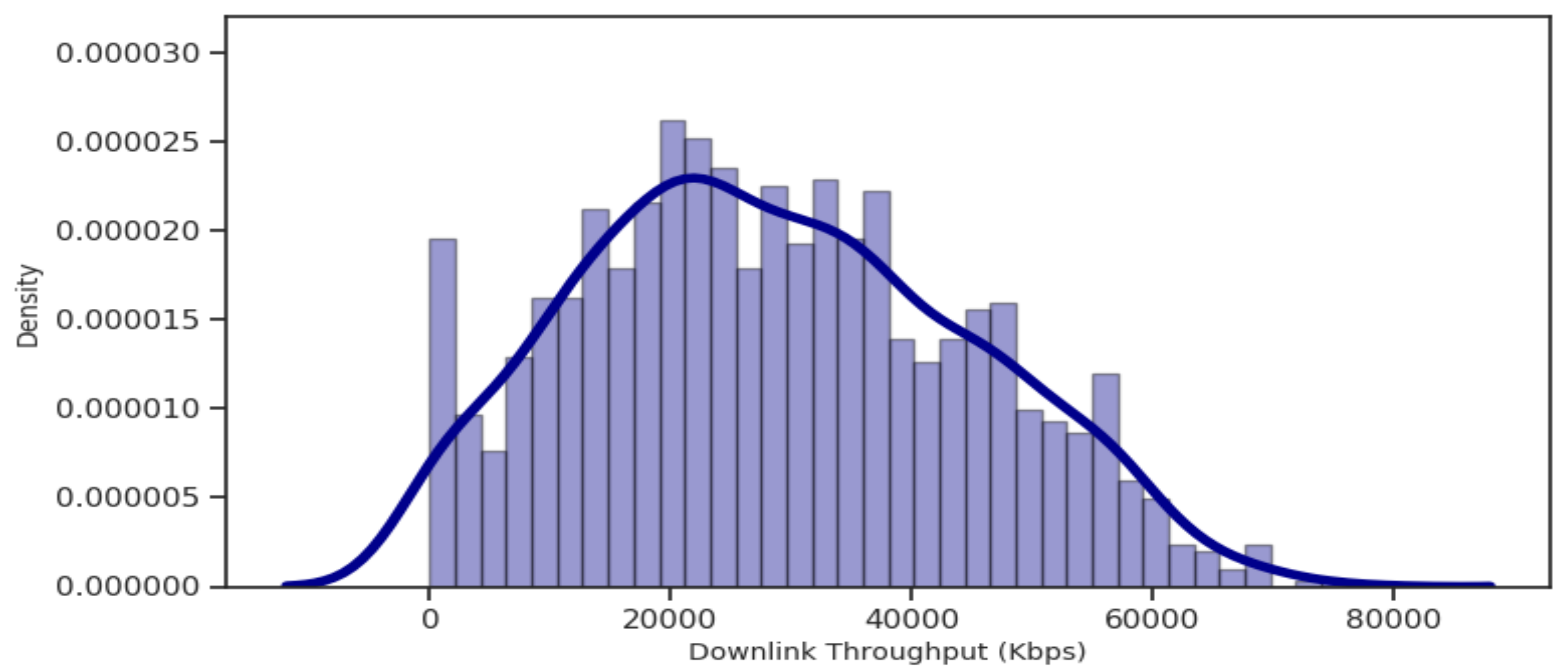

(a) Throughput density plot for the 9-am-trip.

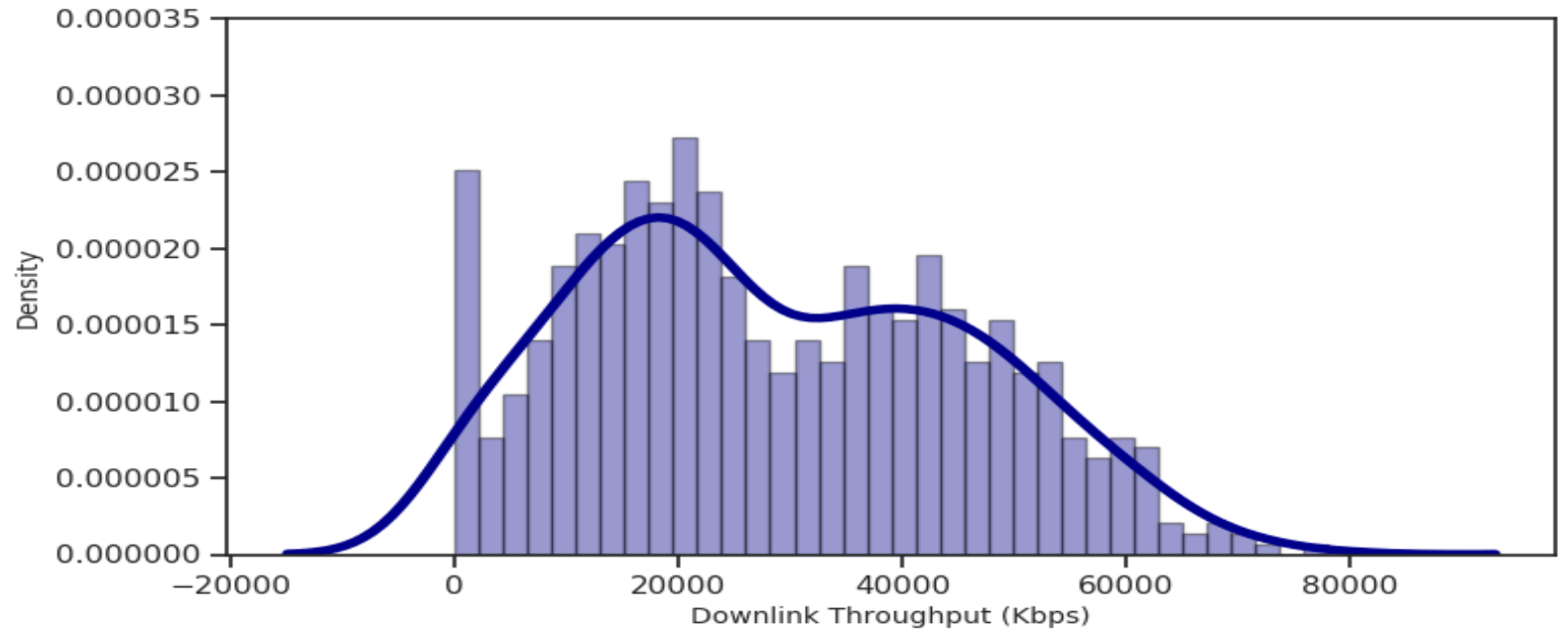

(b) Throughput density plot for the 12-pm-trip.

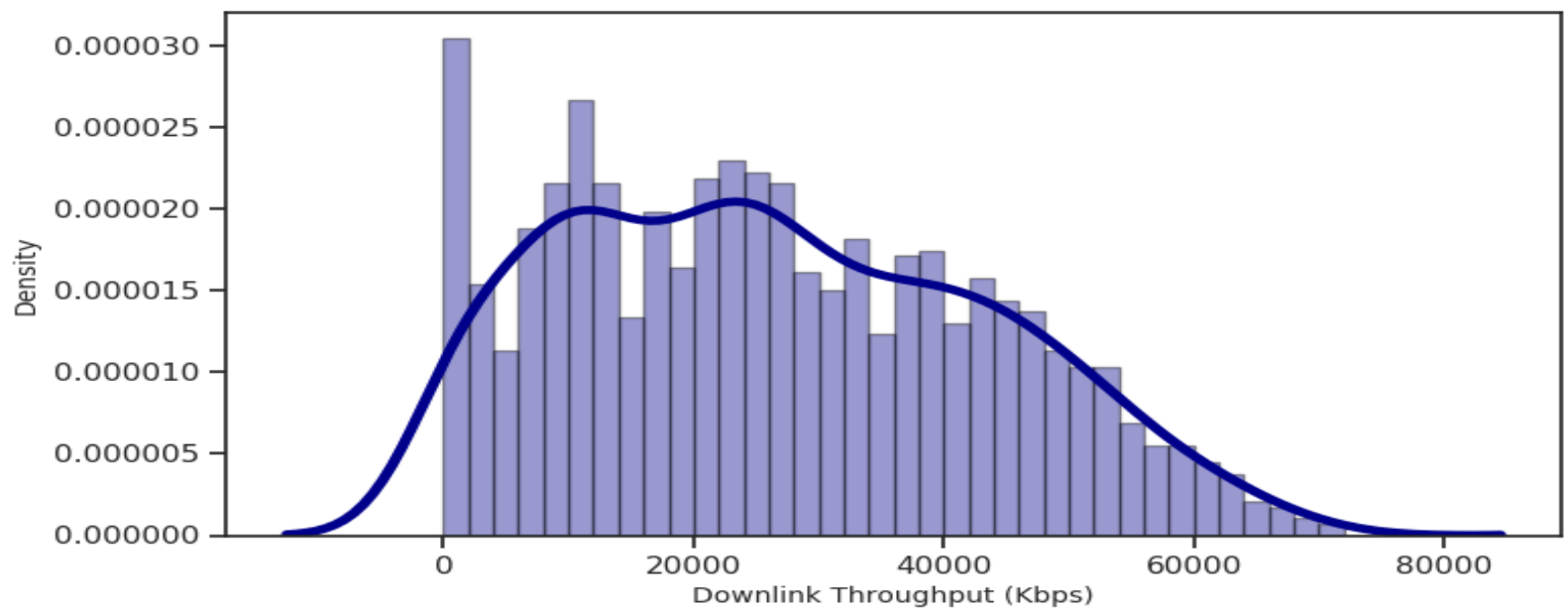

(c) Throughput density plot for the 6-pm-trip.

Figure 3-16: : Density plots for throughput at (a) 9 am, (b) 12 pm, and (c) 6pm. 


\subsubsection{Correlation Analysis}

For correlation analysis, we calculated the Pearson correlation coefficient; this coefficient is used to measure the linear association strength between two variables. Given paired data $\left\{\left\{x_{1}, y_{1}\right\}, \ldots,\left\{x_{n}, y_{n}\right\}\right\}$, Pearson correlation coefficient is computed by the following:

$$
r=\frac{\sum_{i=1}^{n}\left(x_{i}-\bar{x}\right)\left(y_{i}-\bar{y}\right)}{\sqrt{\sum_{i=1}^{n}\left(x_{i}-\bar{x}\right)^{2}} \sqrt{\sum_{i=1}^{n}\left(y_{i}-\bar{y}\right)^{2}}}
$$

where:

- $\quad n$ is the sample size

- $\quad x_{i}$ and $y_{i}$ are individual sample points.

- $\quad \bar{x}$ is the first sample mean defined as: $\bar{x}=\frac{1}{n} \sum_{i-1}^{n} x_{i}$

- $\quad \bar{y}$ is the second sample mean defined as: $\bar{y}=\frac{1}{n} \sum_{i-1}^{n} y_{i}$

The coefficient is calculated by dividing the covariance of two variables by the product of their standard deviations. A correlation coefficient value of 1 means a perfect positive correlation, while a value of -1 means a perfect negative correlation. A value of 0 means there is no correlation at all.

Figure 3-17 shows the correlation matrix between different network parameters and the downlink and uplink throughput. We can see that the RSRP has a strong correlation with both the RSSI and SNR, which is expected since the RSSI is dependent on the RSRP and the RSRP and SNR are proportional to each other. Moreover, the downlink throughput has a medium correlation with the RSRP, RSSI and SNR. This means that we could estimate the throughput if we have the values of these parameters. Furthermore, we can observe a medium correlation between the RSRQ and RSRP, and between the RSRQ and SNR. 


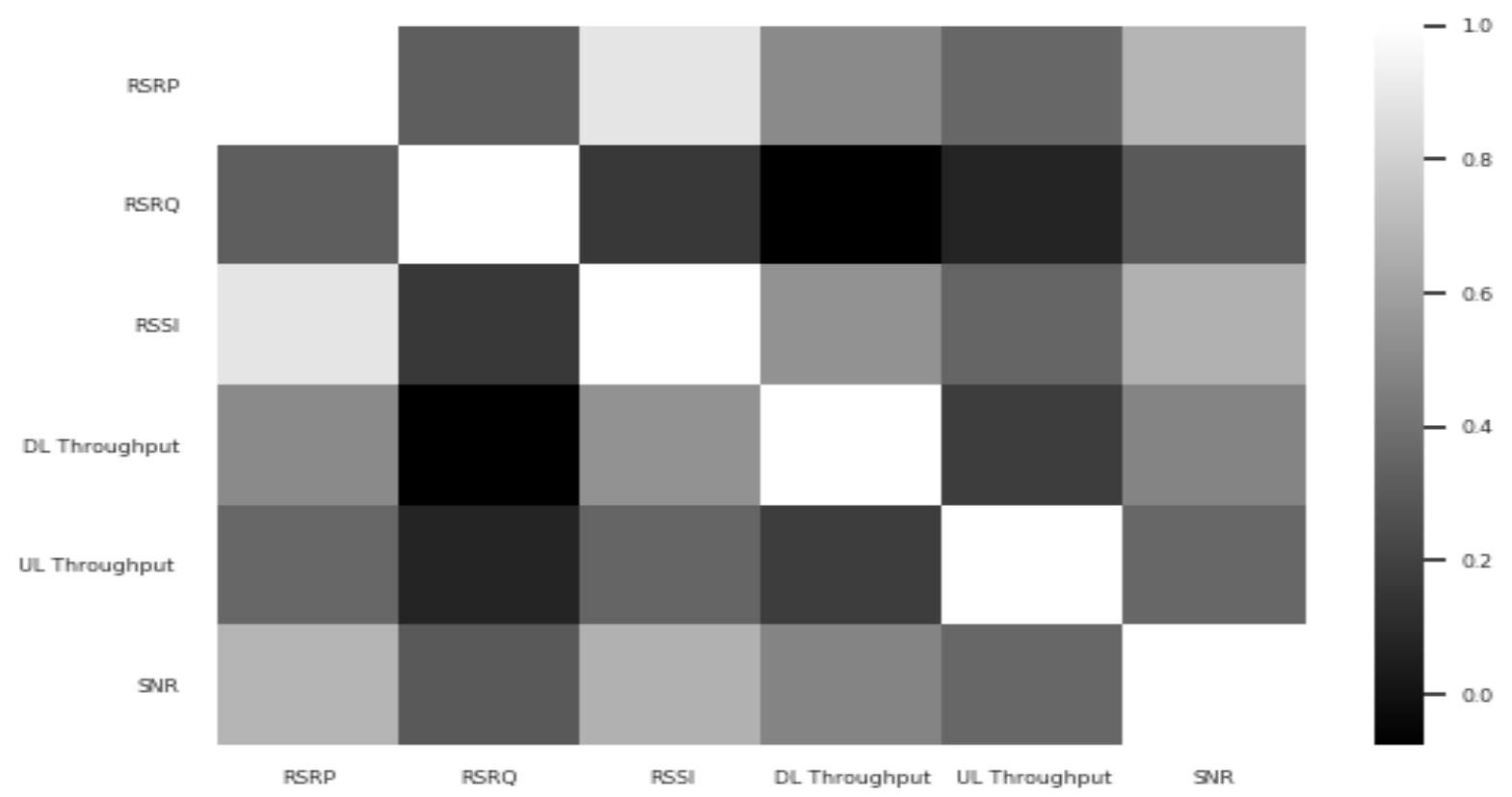

Figure 3-17: Correlation matrix of different features in the dataset.

Figure 3-18 displays the RSRP against the SNR. Consistently with the correlation matrix in Figure 3-17, a linear relationship between the RSRP and SNR can be observed.

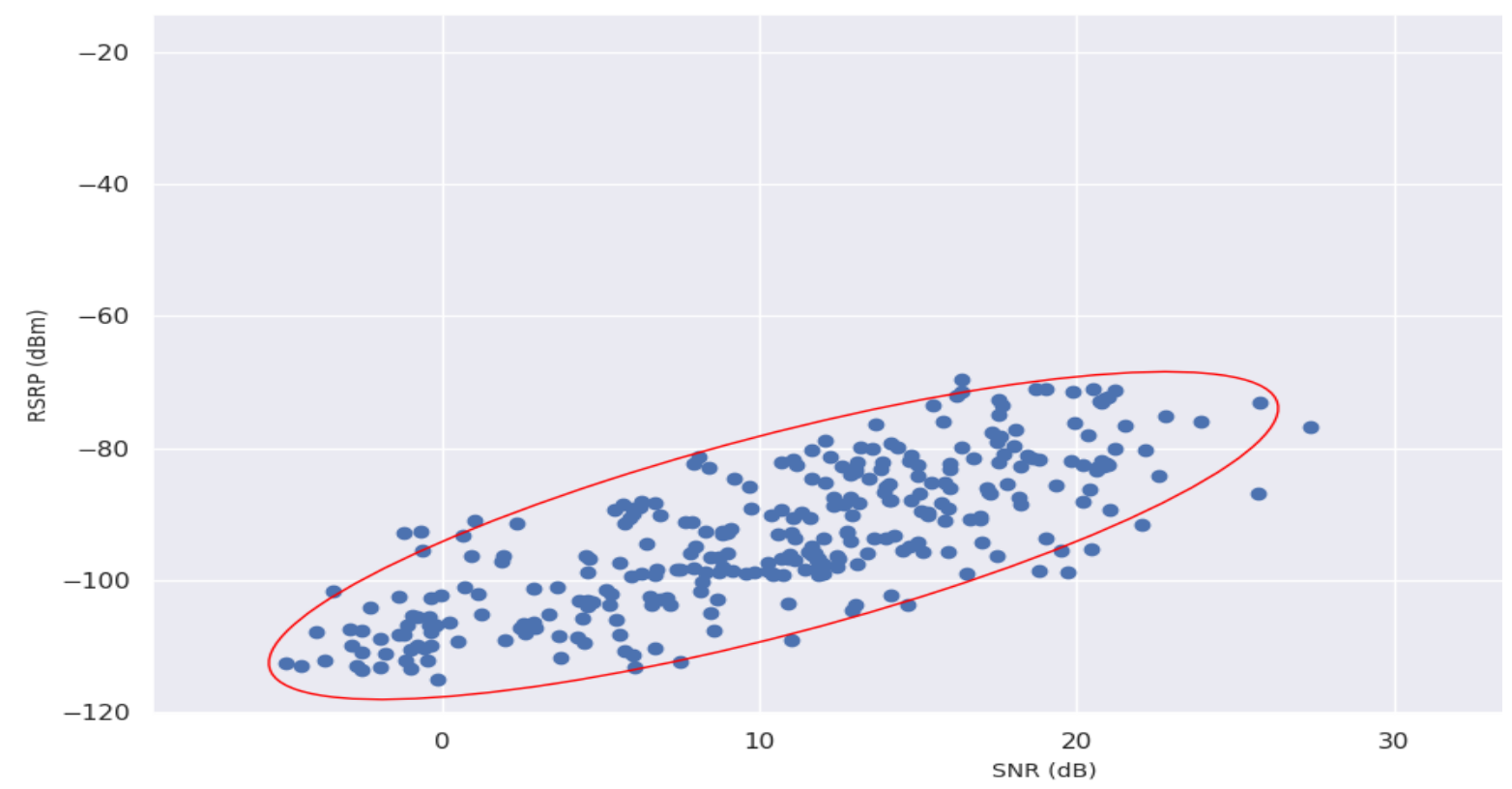

Figure 3-18: RSRP vs. SNR. 
Likewise, Figure 3-19 plots the downlink throughput against the signal strength. We can infer from the plot that the throughput is proportional to the signal strength. This confirms our earlier statement that was based on the correlation matrix.

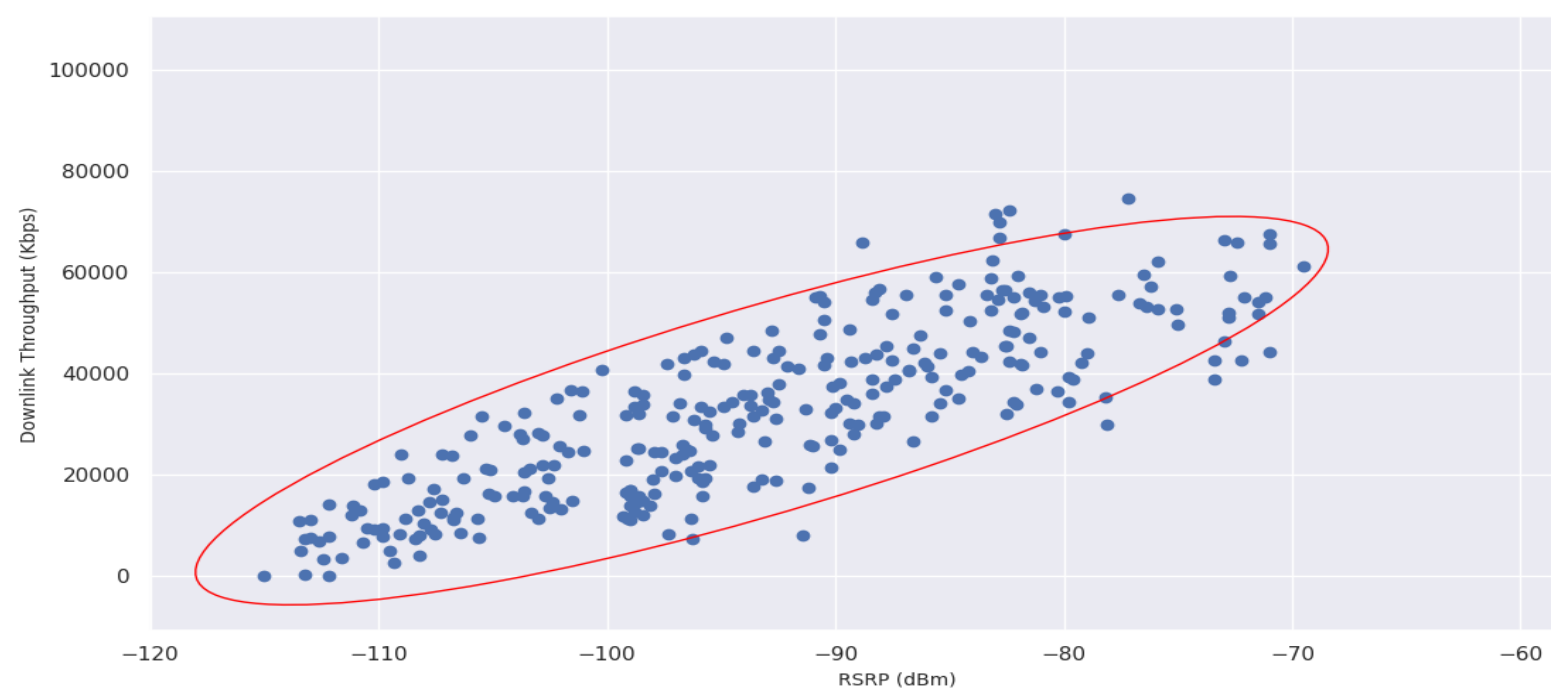

Figure 3-19: Downlink throughput vs. RSRP.

\subsection{Summary}

In this chapter, we presented our data collection goals and metrics, and then explained in detail our measurement setup. Furthermore, we described the dataset attributes and talked about the limitations that we faced during our network survey. Additionally, we explored the data attributes, focusing on the signal strength and throughput variations with time and location, and analyzed the correlation between the data attributes. In the next chapter, we will explain data preprocessing steps that we have done and discuss the process of throughput modelling and prediction using different machine learning algorithms. 


\section{Chapter 4}

\section{Throughput Modelling and Prediction}

In this chapter, we explain the steps we carried out to apply machine learning models, as well as time series forecasting approaches, on the collected data for throughput prediction. In Section 4.1, we present the preprocessing steps that we did to prepare the data for the machine learning models. In Section 4.2, we discuss the different techniques used for feature selection. In Section 4.3 and Section 4.4, we discuss the various machine learning models that we applied for throughput prediction and the hyperparameters tuning process, respectively. In Section 4.5, we describe different techniques for data splitting. In Section 4.5, we explain time series forecasting and the different approaches that we used on the data. And lastly, in Section 4.6, we present the evaluation metrics that were used to assess the performance of the models.

\subsection{Data Preprocessing}

Raw data is often noisy and incomplete; therefore, machine learning models cannot be applied directly on raw data. To ensure the accuracy and efficiency of the machine learning models, some preprocessing steps need to be performed before the data can be fed to the models. In the next subsections, the preprocessing steps used in this work are presented.

\subsubsection{Outliers Detection and Removal}

In statistics, an outlier is a data point that differs significantly from other observations [37]. Outliers can be caused by measurement errors, sampling errors, data entry errors or can occur naturally. The presence of outliers may affect the performance of the machine learning model, as the quality of the data determines the quality of the prediction model. Therefore, it is often desirable to detect and remove outliers before the data is passed to the model.

Outlier detection, also known as anomaly detection, is the process of finding data points that deviate strongly from the rest of the data. There are multiple methods for detecting outliers; we present below the most commonly used methods: 


\section{1- Z-score}

The z-score measures the number of standard deviations above or below the mean a data point is, giving indication of how far from the mean a data point is located [38]. The z-score of a data point can be calculated using the following equation:

$$
\mathrm{Z}=\frac{(\mathrm{x}-\mu)}{\sigma}
$$

where $\mathrm{x}$ is a data point, $\mu$ is the mean of all data points and $\sigma$ is the standard deviation of all data points.

The z-score works well when the data has a Gaussian distribution. The outliers are considered the data points that are located at the tails of the distribution, as they are viewed as too far from the mean to be reasonable. To detect outliers, a threshold is specified for the value of the z-score. Usually, a value of 3 or -3 is specified as the threshold, and a data point is detected as an outlier when its z-score is greater than 3 or less than -3 .

An advantage of the $\mathrm{z}$-score method is that it is very effective if the data has Gaussian distribution. A disadvantage is that it is not practical to use in a high dimensional feature space.

\section{2- The Interquartile Range (IQR) score}

The IQR score is another widely used method for outlier detection. Unlike the z-score, the IQR score does not assume a Gaussian distribution. The value of the IQR depends on the values of the first and third quartiles [39]. The first quartile, denoted by Q1, is the median of the lower half of the dataset, and it holds $25 \%$ of the numbers in the dataset below it. Similarly, the third quartile, which is denoted Q3, is the median of the upper half of the dataset and holds $25 \%$ of the numbers in the dataset above it. The IQR is defined as the difference between the first and third quartiles and is calculated using the following formula:

$$
\mathbf{I Q R}=\mathbf{Q 3}-\mathbf{Q 1}
$$

This technique for outlier detection works as follows: any value that lies above Q3 by $1.5 \times I Q R$ or below Q1 by $1.5 \times I Q R$ is viewed as an outlier and can then be removed from the dataset.

The IQR method has an advantage that it does not depend on a specific distribution, as it uses percentiles. 


\section{3- The Density Based Spatial Clustering of Applications with Noise (DBSCAN)}

Clustering techniques can be useful for detecting outliers. DBSCAN is a density-based clustering algorithm. Clustering algorithms group similar data together into clusters. DBSCAN works by grouping points that are close to each other, based on a distance measure, into clusters [40]. Outliers can then be found as clusters with few data points. DBSCAN classifies data points into three types:

- Core point: a point that contains in its neighborhood a number of points equal to or above the hyperparameter MinPts. The neighborhood radius is defined by the hyperparameter $\varepsilon$.

- Border point: a point is in the same cluster as the core points but are located much further away from the center of the cluster.

- Outlier: a point that does not belong to any cluster.

The DBSCAN method has multiple advantages: it is very effective when the distribution of data is not known, and it is practical to use when the feature space is multidimensional.

On the other hand, before the DBSCAN method is applied, the values of the dataset need to be scaled. Moreover, it is difficult to select the optimal parameters MinPts and $\varepsilon$.

\section{4- Isolation Forests}

Another method used for outlier detection is isolation forests, which is a method based on binary decision trees. Isolation forests depend on the idea that outliers are far away from the rest of the data points [41]. The concept of isolation forests is similar to random forests, although the objective is different. An isolation forest is made up of multiple isolation trees. Each isolation tree is constructed in a top-down approach. The algorithm runs as follows:

First, an attribute is selected randomly from the data, and the algorithms performs a binary split at a point chosen randomly between the minimum and maximum ranges of that attribute. This process is repeated until there is only a single point in each node. The idea is that points in dense regions will need more splits than points in sparse regions. Accordingly, the outlier score of each point is equal to its depth in the isolation tree. The average of the scores from different isolation trees is then calculated to obtain the final outlier 
score. In this approach, outliers are defined as data points that can be isolated easily using random splits [42].

The main advantage of the isolation forest method is that it is effective and robust, and the data does not need to be scaled. Furthermore, the distribution of the data does not need to be known. On the other hand, the disadvantage of this method is that it is difficult to visualize the results.

Since our data attributes have a Gaussian distribution, as we mentioned in Chapter 3, we used the z-score method for outlier detection. More specifically, we used the z-score method implemented in Python's Scikit-Learn library [43] to remove the outliers.

\subsubsection{Filling in Missing Data}

Missing data are one of the most common sources of error in any code, and the machine learning models do not work when the input data contains missing values. A simple way to deal with missing values is to remove them completely, but that would decrease the amount of data significantly and would risk losing valuable information. Accordingly, a more common solution is to impute the missing values. The following are the commonly used imputation techniques:

\section{1- Imputation with Zero/Most Frequent}

Imputation with zero and imputation with most frequent are done by replacing the missing value in a column with zero or with the most frequently occurring value in that column, respectively. This technique is often done for categorical data and has an advantage of being easy and simple to understand and implement. On the other hand, the technique does not preserve the correlations between variables, and might not be very accurate.

\section{2- Imputation with Mean/Median}

Imputation with mean/median is done by replacing the missing values in a column with the mean/median of the other values in that column. Unlike imputation with zero/most frequent, imputation with mean/median only works on numerical data. 
As with the imputation with zero/most frequent technique, imputation with mean/median is also easy and simple to understand and implement. However, it does not preserve the correlations between different data variables.

\section{3- K Nearest Neighbors (KNN)}

$\mathrm{KNN}$ is also a powerful imputation technique. In this technique, $\mathrm{k}$ neighbors are selected based on a certain distance measure and their average is computed and used as an imputation estimate [44]. Imputation by KNN has two hyperparameters that must be selected: the number of nearest neighbors (k) and the distance metric. The distance metric depends on the type of data, whether it is numeric or categorical. Common distance metrics for numeric data are the Euclidean [45], Manhattan [46] and Cosine [47] distances. On the other hand, for categorical data, the Hamming distance [48] is often used, which counts how many times the points in the two data vectors being compared differ, or the number of substitutions needed to convert one of the data vectors into the other.

A major advantage of imputation by $\mathrm{KNN}$ is that it is much more accurate than the imputation by mean, median or most frequent methods. On the contrary, it is sensitive to outliers and can be computationally expensive.

\section{4- Multivariate Imputation by Chained Equations (MICE)}

Another powerful method for replacing missing values is imputation using MICE. This method creates multiple imputations, instead of just one. The idea behind this method is that multiple imputations are more effective than a single imputation, as multiple imputations can more accurately reflect the distribution of the underlying data [49]. The MICE algorithm works by running multiple regression models and estimating each missing data value based on the other observed values. The advantages of this imputation method are that it is flexible, easy to use and can handle different data types.

Since we did not have a large amount of missing values in our dataset, we performed the mean imputation using Pandas library. 


\subsubsection{Feature Scaling}

Feature scaling is often required in machine learning when the data features have different ranges, especially when tested models rely on the distance between the features. Therefore, features with larger range would influence the result more than those with smaller range.

The following are a few more commonly used scaling techniques:

\section{1- Z-score Normalization}

Z-score normalization, also called standardization, is a scaling technique that transforms the distribution of the data, causing it to have a mean of 0 and a standard deviation of 1 . In this technique, each data point is replaced by its z-score. As we mentioned earlier, the z-score can be computed using Equation 4.1.

\section{2- Mean Normalization}

Another feature scaling technique is the mean normalization, which causes the data to have values between -1 and 1 and mean of 0 . Mean normalization can be performed using the following formula:

$$
x^{\prime}=\frac{x-\operatorname{mean}(x)}{\max (x)-\min (x)}
$$

To perform mean normalization, it is necessary to know the maximum and minimum values of the data.

\section{3- Min-Max Scaling}

Min-Max scaling can also be used to scale data features. In Min-Max scaling, the features are scaled between 0 and 1 [50]. The formula for Min-Max scaling is given by the following equation:

$$
x^{\prime}=\frac{x-\min (x)}{\max (x)-\min (x)}
$$

In this work, the z-score normalization method was used.

\subsubsection{Data Binning}

As we mentioned before, raw data tend to be noisy. Data binning or discretization is a preprocessing technique that reduces the effect of noisy data [51]. This technique works by first sorting the data, dividing the data values into bins with smaller intervals, and then replacing their values with a more general value 
calculated for each bin, such as the mean or median. Data binning has a smoothing effect on the data and causes the model to have better generalization power.

There are two main approaches for binning:

- Equal Width Binning: In this approach, each bin contains a specific numeric range. The algorithm divides the data into $\mathrm{k}$ equal-width intervals. The width of intervals is given by the following formula:

$$
\boldsymbol{w}=\frac{(\max -\min )}{k}
$$

- Equal Frequency Binning: This approach divides the data into bins that contain an equal number of points.

Furthermore, there are three approaches for data smoothing through binning:

- Smoothing by bin mean: Where the value of each bin is replaced by the mean of all values in the bin.

- Smoothing by bin median: Where the value of each bin is replaced by the mean of all values in the bin.

- Smoothing by bin boundary: Where the maximum and minimum values in a bin are selected as the bin boundaries, and then the value of the bin is replaced by the closest boundary value.

We applied the equal frequency binning method by aggregating data for every five seconds. Moreover, we used the smoothing by bin mean approach to get the mean of each attribute during the five seconds interval.

\subsection{Feature Selection}

Feature selection is the process of selecting the features that contribute most to the prediction variable. It is an important step in the machine learning pipeline as training a model with irrelevant features could decrease the accuracy of the model and result in erroneous predictions. Moreover, training the model with fewer attributes reduces the complexity of the model, and makes the model simpler and easier to understand. In the following subsections, three commonly used methods for feature selection are discussed. 


\subsubsection{Univariate Selection}

This method performs statistical tests that indicate which features have the strongest relationship with the target variable. The Chi-square test is one example of these statistical tests and is often used to test the association between two variables [52]. Basically, the Chi-square between each feature and the target is calculated, and then features with best Chi-square scores are selected.

The Chi-square is calculated using the following equation:

$$
\boldsymbol{x}_{c}^{2}=\sum \frac{\left(\boldsymbol{O}_{i}-E_{i}\right)^{2}}{E_{i}}
$$

where $\mathrm{c}$ is the degrees of freedom, $\mathrm{O}$ is the observed value and $\mathrm{E}$ is the expected value.

When a feature has a high correlation with the target value, the observed count is far from the expected count. Therefore, the features will have a high Chi-Square value. Having a high Chi-square value means that the target value is dependent on the feature, and thus the feature can be selected for model training.

\subsubsection{Feature Importance}

The importance of each feature in the dataset can be computed using the feature importance property of the model. The idea of feature importance is to calculate a certain score for each feature in the dataset, where higher scores indicate that this feature contributes more toward the prediction of the target variable. Two common techniques that are used for computing feature importance are: model specific feature importance and permutation feature importance.

\section{1- Model Specific Feature Importance}

This technique is implemented by the random forest algorithm in Python's Scikit-Learn library. In this technique, the average reduction in impurity caused by each feature is calculated across all the trees in the forest. Furthermore, the features that cause the nodes to split closer to the root will have a higher feature importance value [53].

\section{2- Permutation Feature Importance}

Permutation feature importance measures the importance of a certain feature by permuting that feature and then measuring the mean decrease in performance. A feature has a high importance value when the model's 
prediction performance decreases when the feature's values are permuted, meaning that the feature contributed to the model's prediction. Along with the feature importance estimate, this technique also gives a measure of uncertainty of that estimate. On the contrary, the main disadvantage of the permutation feature importance is that it becomes computationally expensive when the feature space increases.

\subsubsection{Correlation Matrix with Heatmap}

Correlation is a statistical term that measures the association between variables [54]. A positive correlation between two variables means that when one variable increases, the other increases as well. On the contrary, a negative correlation means that when one variable increases, the other decreases.

Correlation analysis can help with feature selection, as a feature that has a high correlation with the target variable can be selected as input to the model. Furthermore, features with high correlation with each other can be linearly dependent, and therefore have a similar effect on the target variable. In that case, one of the two features can be dropped.

In Chapter 3, we analyzed the correlation matrix of the different data variables and found that the features with highest correlation with the throughput are the RSRP, RSSI, and SNR. In addition, the feature importance property of the random forest model indicated that the most important features for throughput prediction are ranked as follows: RSRP, RSSI, SNR, RSRQ, timestamp, longitude, and latitude. Therefore, we only used these variables as input to the machine learning model and disregarded the remaining data variables.

\subsection{Machine Learning Models}

In order to determine the best model that could be used on the collected data for throughput prediction, we trained and tested various models on the data to compare their results. The evaluation and comparison are presented in Chapter 5. All the models that we used were implemented in Scikit-Learn library in Python. Specifically, the following are the machine learning models that we used in this work:

\section{1- Ridge Regression}


Ridge regression is a variant of linear regression [55], which is a machine learning algorithm that performs regression analysis [56]. Regression models a target value based on the input features. Particularly, regression predicts a dependent variable value based on a given independent variable. In linear regression, this is done by finding a linear relationship between the dependent and independent variables, as shown in Figure 4-1. A simple regression line can be modelled by:

$$
y=B_{0}+B_{1} x
$$

where $\mathrm{x}$ is the input training data and $\mathrm{y}$ is the target variable. During training, the model tries to find the best-fit line to predict the value of $\mathrm{y}$ for a given value of $\mathrm{x}$, by finding the optimal values for the coefficients $B_{0}$ and $B_{1}$. Figure 4-1 shows an example of linear regression with one independent variable.

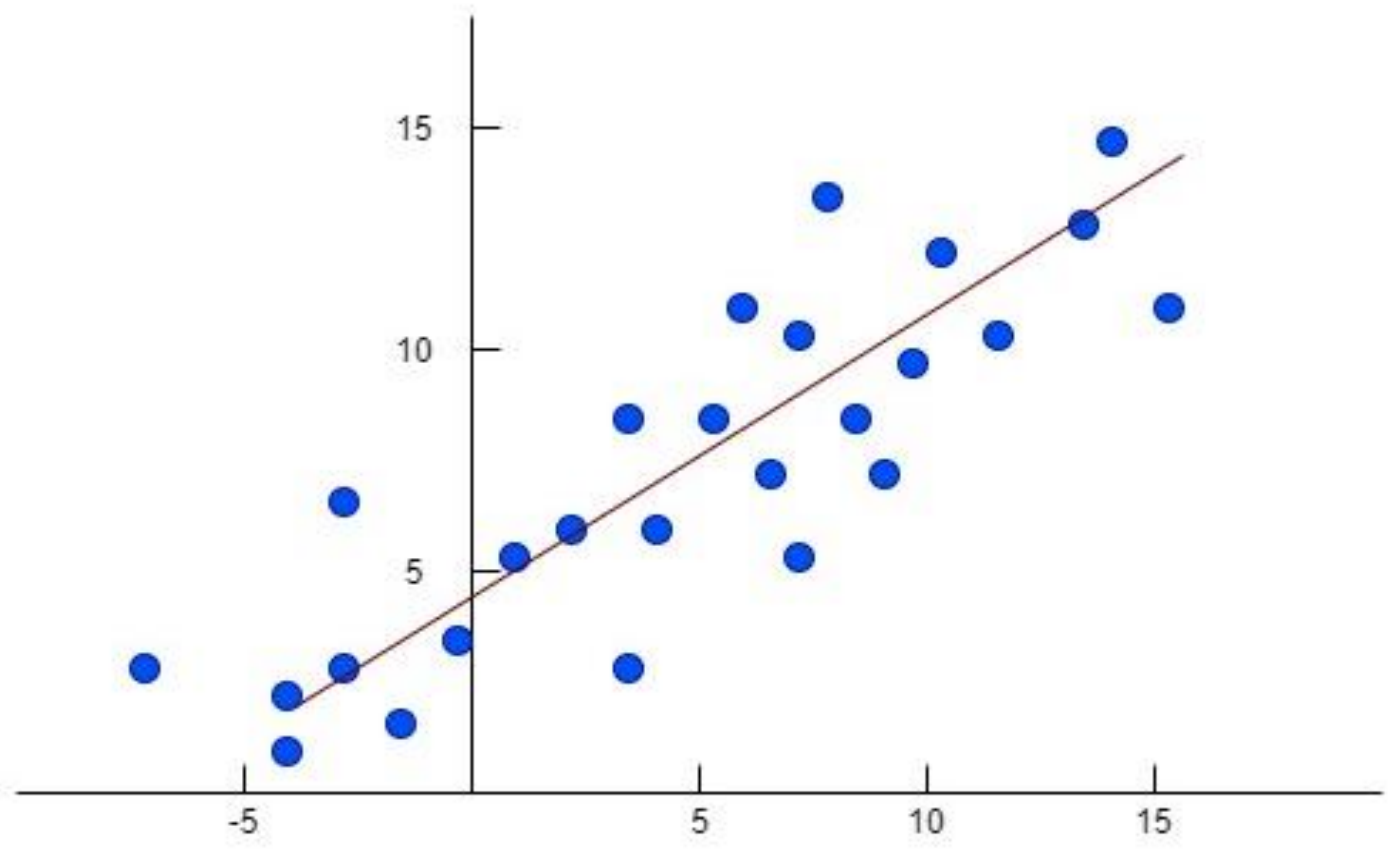

Figure 4-1: Linear regression example.

The task of finding the best-fit line is often done by minimizing the sum of squares of the residuals [57], where a residual is the difference between the observed value and the value fitted by the model. This formula for minimizing the sum of squares is shown below:

$$
\min _{w}\left\|X_{w}-y\right\|_{2}^{2}
$$


Two common problems that occur in machine learning are underfitting and overfitting. Underfitting occurs when the model does not learn enough from the data and cannot capture its underlying structure [58]. This causes the model to have unreliable predictions and low generalization power. On the other hand, overfitting occurs when the model is very flexible, and cannot generalize well from the training data to other unseen data [59]. In other words, the model performs well on the training set, but has a very poor performance on the test set. When there is collinearity between the data variables, i.e., the data variables are highly correlated, the least squares method causes overfitting. Overfitting is a major concern, as it negatively impacts the performance of the model. To prevent overfitting, regularization techniques are often used to reduce the complexity of the model. There are two variants of linear regression that perform regularization:

- Lasso Regression: Performs L1 regularization [60], where the least squares method is modified to also minimize the absolute sum of $\beta$ through the shrinkage hyperparameter $\lambda$, as illustrated in the following formula:

$$
\operatorname{argmin}_{\beta \in \mathbb{R}^{P}}\|y-X \beta\|_{2}^{2}+\lambda\|\beta\|_{1}
$$

where $\beta$ is the coefficient vector $\left[B_{0}, B_{1}\right]$.

- Ridge Regression: performs L2 regularization, where the least squares method is modified to also minimize the squared absolute sum of $\beta$ through the shrinkage hyperparameter $\lambda$, as shown in the following formula:

$$
\operatorname{argmin}_{\beta \in \mathbb{R}^{P}}\|y-X \beta\|_{2}^{2}+\lambda\|\beta\|_{2}^{2}
$$

where $\beta$ is the coefficient vector $\left[B_{0}, B_{1}\right]$.

We first trained a linear regression model for throughput prediction, but the model's performance was not satisfactory. This is attributed to overfitting. To prevent overfitting, we then trained a ridge regression model and the model's performance was significantly better than the linear regression's one.

\section{2- Regression using KNN}

The $\mathrm{KNN}$ is a supervised learning algorithm that is commonly used in machine learning tasks for classification and regression due to its simplicity and applicability in lots of real-world problems. It is a 
non-parametric algorithm that does not make any assumptions about the data distribution. The KNN algorithm predicts the target value based on the similarity between different points. To find similar points, the algorithm uses a distance measure such as Euclidean distance, Hamming distance, Manhattan distance and Minkowski distance [61].

For every point $P_{i}$ it takes as input, it finds the nearest k neighboring points of $P_{i}$ using one of the distance measures mentioned above, and then predicts the output of $P_{i}$ based on the value of its nearest neighbors. In case of classification, the output label of $P_{i}$ would be equal to the majority vote of its k neighbors. In case of regression, the output target value of $P_{i}$ would be the mean of its nearest k neighbors. Accordingly, for the algorithm to compute the output of any point, it needs to have available all the other points. As a result, all the training data is stored and used in the testing stage. This process takes up memory and disk space and causes the testing to be slower. Figure 4-3 displays two examples of KNN for regression and classification, where the number of $\mathrm{k}$ is 3 .
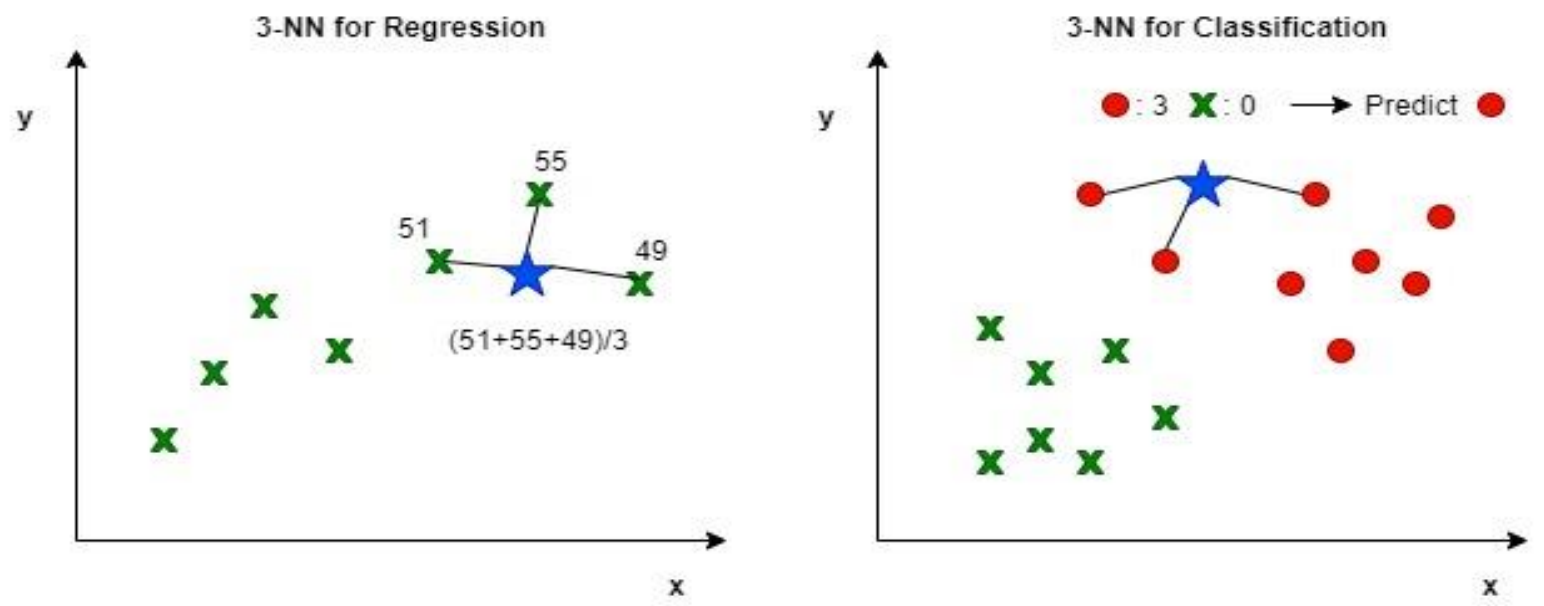

Figure 4-2: KNN Example for (a) regression, (b) classification.

Other than taking up space and memory, the algorithm has another disadvantage; it requires feature scaling as distance measures like the Euclidean distance are very sensitive to the magnitudes of the data.

The advantage of the KNN algorithm is that its training phase is significantly faster than other machine learning algorithms, as it is not required to train the model for generalization. 


\section{3- Support Vector Machine Regression (SVR)}

Support vector machine (SVM) is a supervised learning algorithm that can be used for both classification (SVC) and regression (SVR). SVM uses a technique called the kernel trick to transform the data variables into a higher dimensional space, and then finds the optimal boundary between the possible outputs based on these transformations [63]. Before explaining how SVM works, we should first clarify some definitions. A hyperplane is a separation line that helps classify different data points in the case of classification, or a line that fits the data in the case of regression. Additionally, there are two other lines in SVM called the boundary lines, and their function is to create a boundary. In classification, these boundary lines separate the two classes. On the other hand, in regression, the boundary lines bound the points that are considered for prediction between them. Another term we need to define is the support vectors, which are points that are closest to the boundary, and can even lie on it.

In the case of classification, the SVC uses the kernel trick to convert a problem with data that is nonseparable into data that is separable. For example, if there are two classes in the data and the classes are not linearly separable, the kernel trick can add one more dimension to the data, and then the algorithm can find a hyperplane that could separate the two classes in the higher dimensional space.

In the case of regression, SVR uses the kernel trick to map the input into a higher dimensional feature space and then constructs a linear regression model in this higher dimensional space [62].

In SVR, the distance between the hyperplane and the boundary line is denoted by $\varepsilon$. The goal is to find the optimal value of $\varepsilon$ that the support vectors lie within that boundary line. Figure 4-3 illustrates the concept of SVR, where the hyperplane is shown by the solid line, and the boundary lines are shown by the dashed lines in the figure.

The linear function is given by:

$$
y=w x+b
$$

where $y$ is the target variable, $x$ is the input variable, $w$ is the weight coefficient and $b$ is the bias. 
The optimization function works to maximize the margin by minimizing the squared sum of the weight coefficients to ensure the function is as flat as possible, and it is given by the following formula:

$$
\frac{1}{2}|w|^{2}
$$

The constraint that all of the residuals are less than $\varepsilon$ is ensured by the following rule:

$$
\forall_{n}:\left|y_{n}-\left(w x_{n}+b\right)\right| \leq \varepsilon
$$

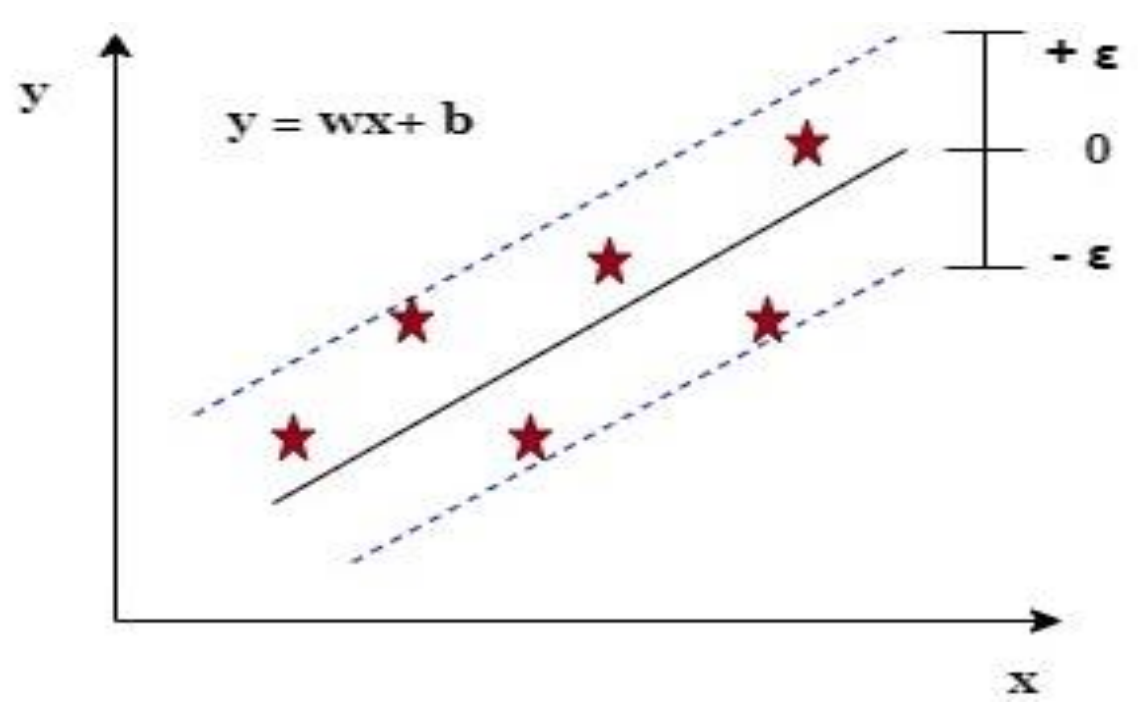

Figure 4-3: SVR Algorithm.

\section{4- Random Forest for Regression}

Random Forest is a type of ensemble learning method, where a group of weak models are combined to form a strong model [64]. It is a supervised learning algorithm that is constructed through an aggregation of decision trees, where each tree is trained on a subset taken from the data. Generally, the larger the number of decision trees, the more accurate the prediction results are.

Decision trees use a tree-like graph to formulate rules and make predictions based on these rules. In decision trees, each node represents a feature, each branch represents a decision, and each leaf represents an output. The goal is to create a tree for all the features in the dataset and use it to produce a different output at each 
leaf. The output that is produced depends on the set of decisions made by the tree as it processes the input feature vector.

The decision trees are constructed in a top-down approach that is also known as recursive binary splitting. The construction begins at the top of the tree where the entire dataset is available, and then splits the predictor space into two new branches down the tree. During construction, an important step is to identify the feature that will be represented by the root node at each level. For efficient prediction, we need to split the nodes at the most informative features [65]. In the case of classification, a commonly used metric that is used in this step is the information gain [65], which is the decrease in entropy. Entropy is a measure of the impurity in the data samples and is computed by Equation 4.14. Whenever we split a node, the entropy changes. Information gain computes the difference between entropy before and after the split of node.

$$
E(t)=-\sum_{i=1}^{m} p(i \mid t) \log _{2} p(i \mid t)
$$

where $p(i \mid t)$ is the number of the samples that belong to class $\mathrm{c}$ for a certain node $t$.

In the case of regression, however, another metric is needed to accommodate for the continuous variables. A metric that could be used in regression is weighted mean square error (MSE), which is given by:

$$
\operatorname{MSE}(t)=\frac{1}{N_{t}} \sum_{i \in D_{t}}\left(y^{(i)}-\widehat{y}_{t}\right)^{2}
$$

where $N_{t}$ is the number of training samples at node $t, D_{t}$ is the training subset at node $\mathrm{t}, y^{(i)}$ is the actual target value, and $\hat{y}_{t}$ is the predicted target [65].

Decision trees are widely used due to their high accuracy and stability. Unlike linear models, they are capable of mapping nonlinear relationships within the data attributes.

As we mentioned before, random forest is a collection of decision trees. It works by randomly picking a number of sub-samples from the data samples to build each tree. Then, a number of features are selected 
randomly from all the features to ensure that the trees are not highly correlated. To make a prediction, each tree makes a vote by predicting the target value. The forest then takes the average of all the votes by the different trees in the forest. The algorithm is illustrated in Figure 4-4.

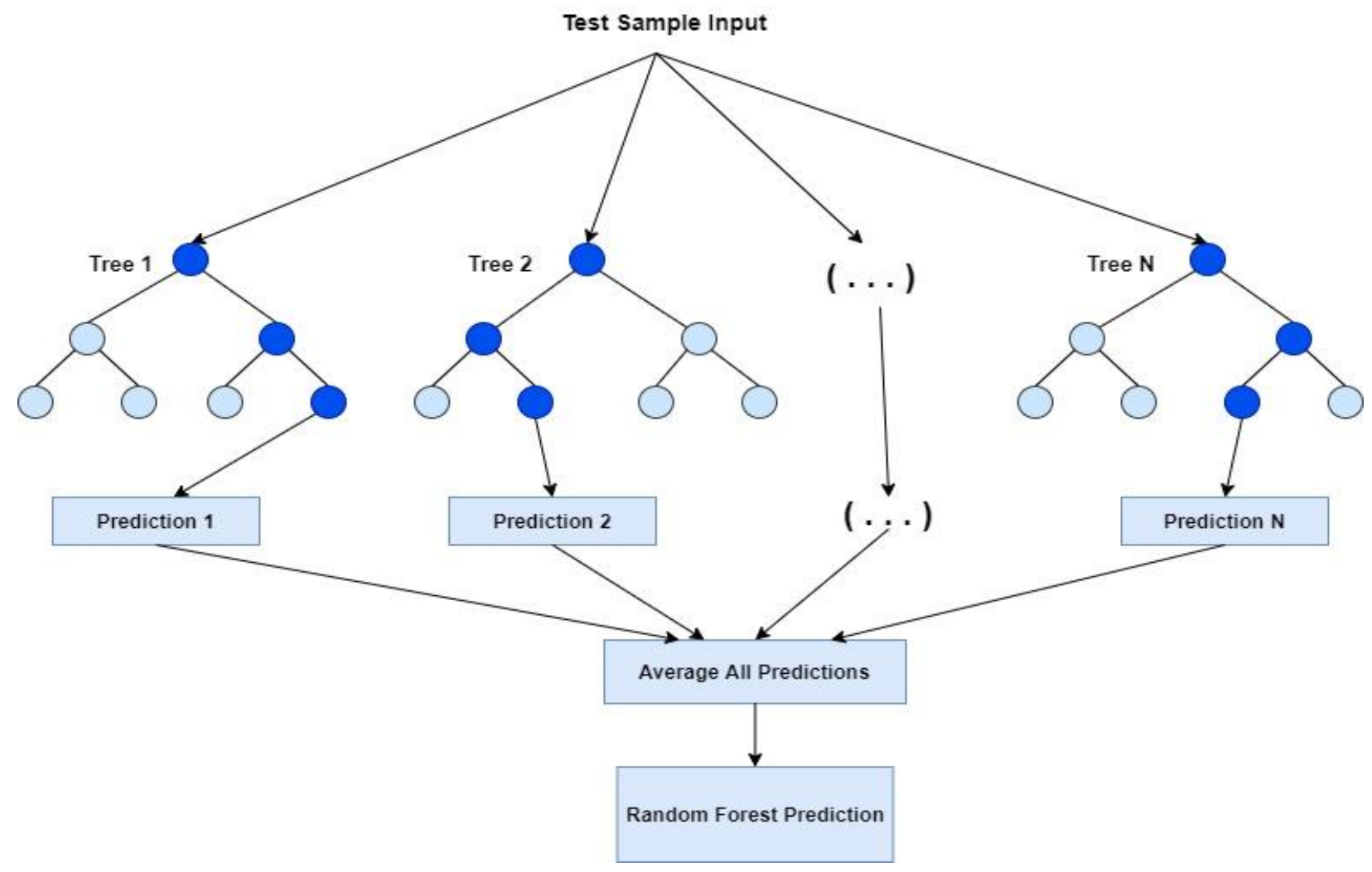

Figure 4-4: Random forest algorithm structure.

There are numerous advantages to the random forest algorithm. It is a robust model that can handle datasets with high dimensionality. Moreover, the model is very useful in data exploration, as it has a property called feature importance that shows the importance of each feature to the prediction, which is useful for feature selection. Additionally, the model requires less data cleaning than many other modeling techniques and it does not make any assumptions about the data distribution.

\subsection{Hyperparameters Tuning}

Hyperparameters are configurable parameters that define the model architecture and govern the training process [66]. They are tuned by training the model, testing it and examining the error, and then adjusting 
the parameters. Moreover, hyperparameters are not learned automatically by the model, they have to be set manually. Various methods have been proposed for hyperparameters tuning. The following are the most used:

\section{1- Grid Search}

In this method, the set of hyperparameters are defined and then an extensive search is performed to search through all combinations of hyperparameters to find the optimal one [67]. Moreover, the method determines the optimal hyperparameter by measuring the performance using cross-validation. The grid search is a simple, easy to use method, but it can be computationally expensive when the data has a high dimensional space.

\section{2- Random Search}

Unlike grid search, where all different hyperparameter combinations are tested, this method pulls a set of random values from the hyperparameter space until the optimal value is found [68]. The random search method has a higher speed and performance level than the grid search. However, a disadvantage of this method is that it is not affected by previous selections when choosing the next set of hyperparameters.

\section{3- Bayesian Optimization}

In this method, a probabilistic model is constructed between the hyperparameter values and the performance measure using the test data [69]. Additionally, different hyperparameters combinations are tested and the model is adapted based on the evaluation of these combinations.

Furthermore, different machine learning algorithms require different hyperparameters to be tuned. For each algorithm below, we discuss the hyperparameters that need to be optimized.

- Ridge Regression: The hyperparameter that is used in ridge regression is the $\alpha$, which corresponds to shrinkage hyperparameter $\lambda$ we described earlier. A larger value of $\alpha$ reduces the model complexity and prevents overfitting. However, after a certain point, increasing the value of $\alpha$ may 
lead to underfitting. Hyperparameter tuning in ridge regression involves balancing the regularization strength in order to achieve the best possible model performance.

- KNN for Regression: Choosing the value of $\mathrm{k}$ affects the performance of the model. Increasing the value of $\mathrm{k}$ reduces the noise and leads to smoother predictions but may cause underfitting. As with ridge regression, the idea of hyperparameter tuning in $\mathrm{KNN}$ regression is to find the optimal value of $\mathrm{k}$ that improves the performance of the model.

- SVR: The hyperparameters used in SVR training are the kernel function, $\varepsilon$, and the regularization parameter $\mathrm{C}$, which penalizes misclassification and margin errors. As there are multiple combinations of these hyperparameters, they are often tuned using the grid search. This approach is based on the concept of exhaustive search; it tries out different hyperparameter combinations and determines the optimal value of each hyperparameter.

- Random Forest for Regression: Hyperparameters in a random forest include the number of decision trees in the forest and the maximum number of features considered by each tree for splitting a node. Increasing the number of decision trees improves the accuracy of the model as it considers the votes from various trees, but it can be computationally expensive. Moreover, increasing the maximum number of features considered by each tree for splitting a node can lead to a higher model performance as it causes the trees to have more features to select from the optimal split, but this can cause overfitting

\subsection{Data Splitting}

To effectively evaluate the performance of machine learning models, cross-validation is commonly used. In cross-validation, the dataset should be split into two subsets: a training set and a test set [70]. The training set is the partition of the dataset that the model is trained on. The test set is the partition of the dataset that the model has not seen before. Both the training set and the test set consist of some input features and a target variable. The model is fit on the features and target variable from the training set and then the fitted 
model is used to predict the target variable for the features in the test set. The test set should be large enough to produce statistically meaningful results and should be representative of the entire dataset.

The dataset split ratio depends on two factors: the number of samples in the dataset and the type of model that is trained, as some models require a large amount of data to train on.

There are three main methods for performing cross-validation: the holdout method, the k-fold crossvalidation and the leave-one-out cross-validation.

- Holdout Method: This approach of this method is to remove a subset of the dataset and then using it to evaluate model trained on the rest of the data [71]. The error is an indication of how well the model is going to perform on the test set. A common split ratio is $70 \%-30 \%$, where the training set consists of $70 \%$ of the dataset and test set consists of $30 \%$ of the dataset.

- K-Fold Cross-Validation: In this approach, the dataset is divided into k subsets. Next, the model is trained on all subsets except for one, and then the model is evaluated on that one subset that was not used in training. This process is repeated $\mathrm{k}$ times, where each time a different subset is used for evaluation [72]. Thus, k-fold cross-validation performs the holdout method k times. This approach is commonly used in small datasets to prevent overfitting, as it improves the generalization power of the model.

- Leave-One-Out Cross-Validation: This approach is a special version of k-fold cross-validation. In leave-one-out cross-validation, the number of folds is equal to the dataset samples [73]. Furthermore, the model is trained repeatedly, where each time one sample is selected as a test set, and all the other samples are selected as a training set.

We trained and evaluated the models using the hold-out method with various data split ratios and reached the best performance with a split ratio of $70 \%$ training set and $30 \%$ test set.

\subsection{Time Series Forecasting}

A time series is a collection of observations taken at consecutive points in time. The aim of time series forecasting is to build a model to forecast future values of a time series based on the previous values. In 
particular, the observations of a time series at time $t$ are used to forecast the future values of it at some future time $t+h$ [74]. Unlike regression predictive modelling, a time series adds an explicit order dependence between the observations. Various techniques and mechanisms have been proposed for time series forecasting. In our work, we used Autoregressive Integrated Moving Average (ARIMA) and Long Short-Term Memory (LSTM) models [76].

\subsubsection{Autoregressive Integrated Moving Average (ARIMA)}

An ARIMA model is a class of statistical models and is one of the most commonly used techniques for time series forecasting. Autoregressive models use a linear combination of the current observation and a number of lagged observations to forecast future values of a variable. On the other hand, moving average models use a linear combination of the residual errors to predict the forecast error at the next time step. ARIMA models combine both approaches into one model to perform time series forecasting [75].

ARIMA models require the time series to be stationary. A stationary time series is one whose statistical properties are not dependent on the time at which the series is observed [75]. One approach that could convert a non-stationary time series to a stationary one is to compute the differences between successive observations. This approach is called differencing and is often an important step in time series forecasting using ARIMA models. Furthermore, ARIMA models have three parameters, namely $p, d$, and $q$, where $p$ is the number of lag observations, $\mathrm{d}$ is the degree of differencing, and $\mathrm{q}$ is the size of the moving average window, also called the order of the moving average.

\subsubsection{Long Short-Term Memory (LSTM)}

LSTM is a type of recurrent neural networks (RNNs) [77] that is used in the field of deep learning. Unlike feedforward neural networks, recurrent neural networks have feedback connections. These feedback connections make the recurrent neural networks capable of handling sequence dependencies. LSTM networks use special units in addition to the standard units of the RNN networks in order to capture long term temporal dependencies. The architecture of LSTM networks includes a memory cell, which is used to 
maintain information for long periods of time. The memory cell consists of four gates: forget gate, input gate, state update gate, and output gate. The input and state update gates are responsible of feeding information into the memory cell, while the forget gate is used for resetting the memory cell. The output gate determines how the output of the memory cell affects other LSTM cells [78]. Figure 4-5 shows the LSTM structure, where $i_{t}$ is the input gate, $f_{t}$ is the forget gate, $o_{t}$ is the output gate, $\sigma$ and tanh correspond to the activation function, $c_{t}$ is the state of the memory cell and $h_{t}$ is the output of the cell.

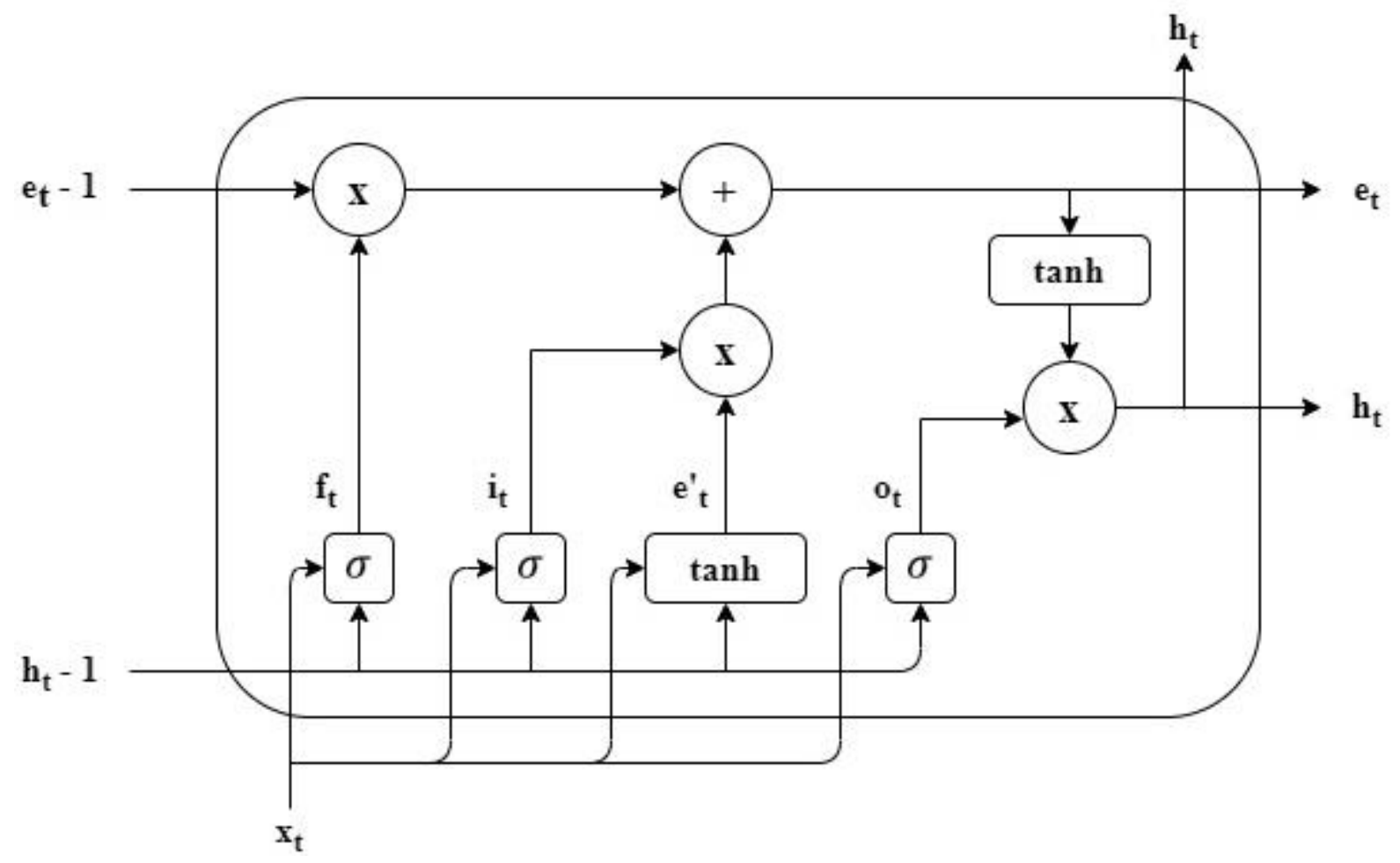

Figure 4-5: Structure of LSTM.

LSTM models can be used for time series modelling and forecasting. For LSTM models to produce accurate predictions, they require very large amounts of data. Additionally, LSTM models can have an additional dropout [79] layer which helps prevent overfitting by ignoring a number of randomly chosen neurons during training. 


\subsection{Evaluation Metrics}

We evaluated the performance of the models using two well-known metrics: the $R^{2}$ score and the root mean square error (RMSE). In the following subsections, the two metrics are described.

\subsection{1 $R^{2}$ score}

Also called the coefficient of determination, the $R^{2}$ score is a goodness-of-fit measure for regression models. It indicates the percentage of the variance in the dependent variable that can be explained by the independent variables [80]. The $R^{2}$ score measures the strength of the relationship between your model and the dependent variable on a convenient $0-100 \%$ scale.

The $R^{2}$ score is computed by:

$$
R^{2}=1-\frac{\sum_{i=1}^{N}\left(y_{i}-\widehat{y}_{i}\right)^{2}}{\sum_{i=1}^{N}\left(y_{i}-\bar{y}_{i}\right)^{2}}
$$

where $\mathrm{y}$ is the actual value, $\widehat{y}$ is the predicted value and $\bar{y}$ is the mean of all y values. The $R^{2}$ score has a range from $0-1$, where 0 indicates that the model doesn't explain any of the variability of the response data around its mean, and 1 indicates that the model explains all the variability of the response data around its mean. Accordingly, a higher $R^{2}$ score specifies that the model fits the data better.

\subsubsection{Root Mean Square Error (RMSE)}

The RMSE is the standard deviation of the prediction errors, also called the residuals [75]. In Figure 4-6, we can see an example of a fitted linear model, where the residuals are the vertical lines showing the difference between the actual data values and values predicted by the model.

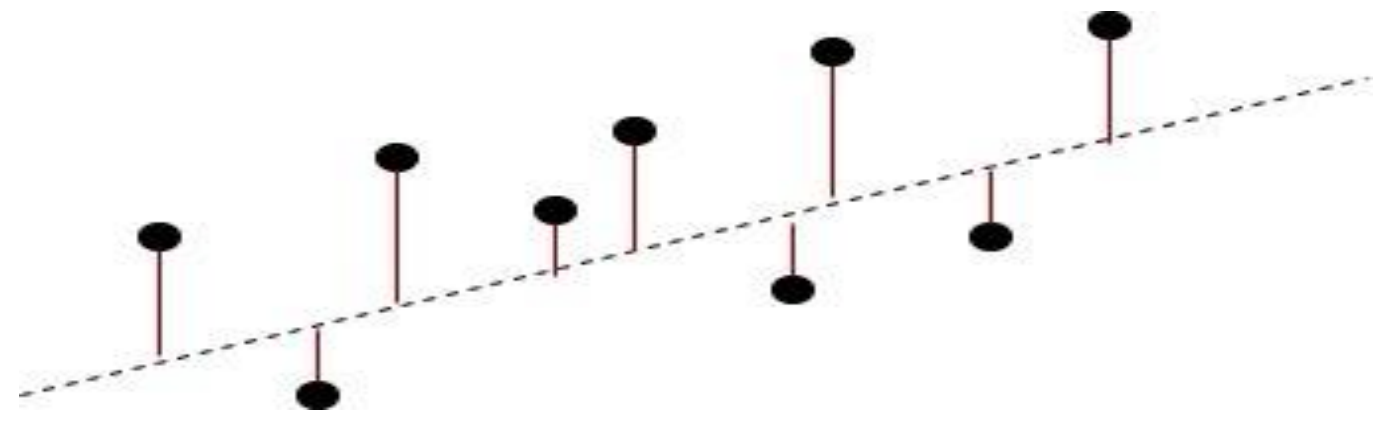

Figure 4-6: Residuals in a linear model. 
The RMSE is used to measure how the residuals are dispersed. Moreover, it can be used to compare the prediction errors of different models to determine the model with the highest performance on the data.

The RMSE can be calculated by the following equation:

$$
R M S E=\sqrt{\frac{\sum_{i=1}^{N}\left(\text { Predicted }_{i}-\text { Actual }_{i}\right)^{2}}{N}}
$$

Where Precidted $_{i}$ corresponds to the model predictions, Actual $_{i}$ corresponds to the actual values of the data samples, and $\mathrm{N}$ is the number of samples. Lower RMSE values indicate that the model fits the data better

\subsection{Summary}

In this chapter, we described the preprocessing steps and explained how we performed feature scaling. Moreover, we discussed the machine learning models that we trained and the hyperparameters tuning that we have completed. Furthermore, we explained different time series forecasting methods and talked about the evaluation metrics that we used to evaluate the performance of our models. In the next chapter, we will present the results of training and testing the models for throughput prediction and compare them based on the evaluation metrics that we mentioned in this chapter. 


\section{Chapter 5}

\section{Results and Discussion}

In this chapter, we present the results of training different models on the collected dataset for throughput prediction. In Section 5.1, we describe the experimental setup for our problem. In Section 5.2, we illustrate the performance of each machine learning model and explain how different hyperparameters affect the model performance. Moreover, we perform a comparative analysis between the different trained models. In Section 5.3, we analyze the performance of the time series forecasting models. Lastly, in Section 5.4 we discuss the results and describe different ways to improve the performance of our models.

\subsection{Experimental Setup}

In this section, we explain how we collected and prepared the data for applying machine learning algorithms for throughput prediction. Additionally, we describe the platform on which we performed data preprocessing and machine learning training and testing.

\subsubsection{Data Collection and Preparation}

As we mentioned in Chapter 3, the data was collected on a public transit bus in Kingston, Ontario, using two Android smartphones: Samsung Galaxy S9 and Samsung Galaxy S10e. The measurements were taken at three different times of the weekday, namely $9 \mathrm{am}, 12 \mathrm{pm}$ and $6 \mathrm{pm}$. After the data collection was completed, we combined the data from both phones using the mean of each measurement. The reason for this step was to reduce the variations and effects of noise in the data. To prepare the data for the machine learning models, we performed multiple preprocessing steps. Outliers were removed using the z-score method, missing values filled in using imputation with mean, then we scaled the features using z-score normalization and performed equal frequency data binning to further reduce the effect of noise. Subsequently, we selected the features to use for training by analyzing the correlation matrix. The features selected were the RSRP, RSSI, RSRQ, SNR, timestamp, longitude, and latitude. To train the machine 
learning models, we used the holdout cross-validation technique, where we split our dataset into $70 \%$ training set and 30\% test set. Figure 5-1 illustrates the pipeline that we used for throughput prediction.

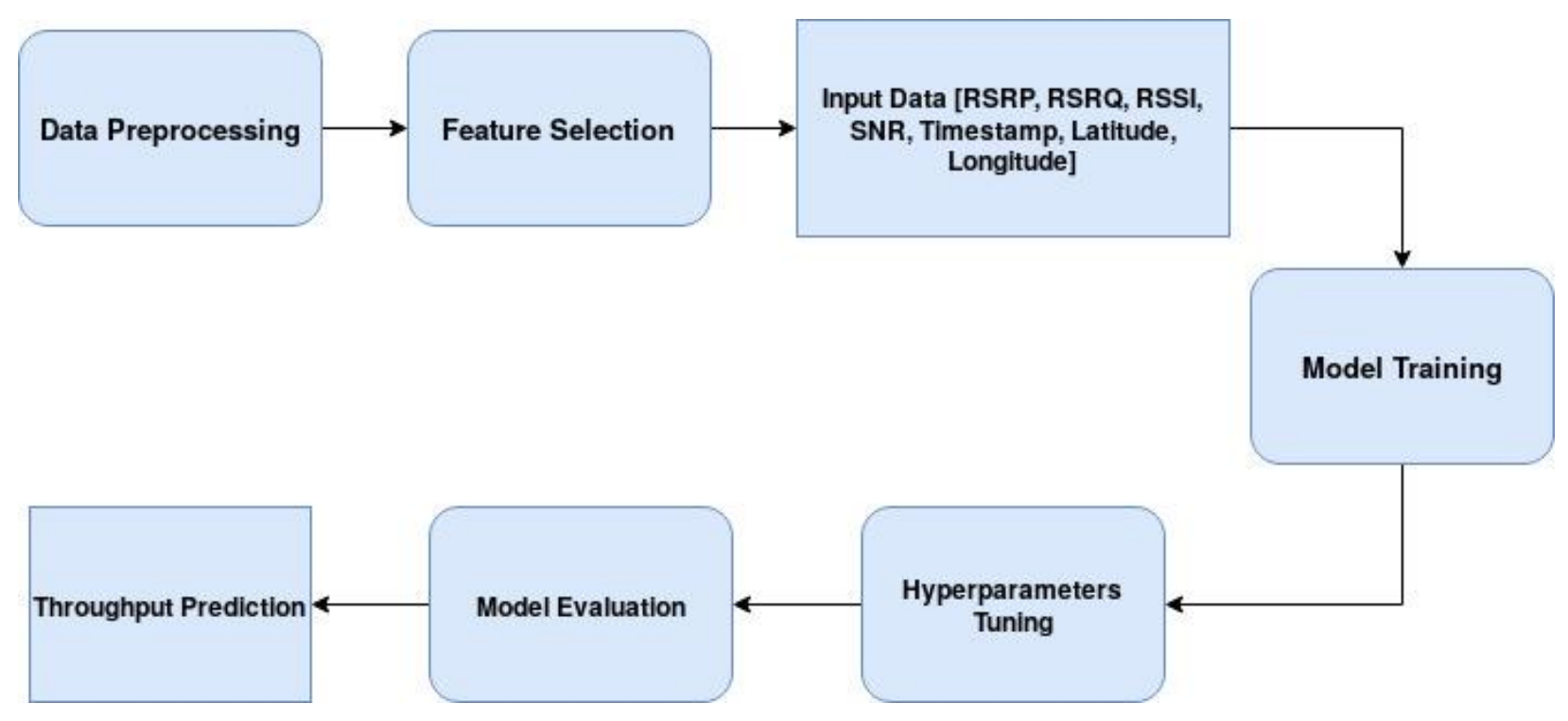

Figure 5-1: Throughput prediction pipeline.

\subsubsection{Platform Used}

For preprocessing the data and training the machine learning models, we used an ASUS computer, INTEL® CORETM i5-7200U Processor and a 4 GB RAM. Moreover, we used Python 3 on a Linux operating system. Scikit-Learn [81], Numpy [82], Pandas [83] and Matplotlib [84] libraries in Python comprise the machine learning framework.

\subsection{Results of Machine Learning Models}

As mentioned in the Chapter 4, we trained several machine learning models on the collected data for throughput prediction. To assess the learning model performance, we used both evaluation metrics mentioned in Chapter 4, the $R^{2}$ score and root mean square error (RMSE). Moreover, we performed a comparative analysis between the different models to determine the best predictor for our problem. The test results for each model are presented in the following subsections. 


\subsubsection{SVR}

The SVR algorithm contains various hyperparameters, namely $\mathrm{C}$, which is the regularization parameter, the kernel function and $\varepsilon$. As there are various possible combinations of hyperparameters, we took advantage of the grid search to determine the optimal combination. Based on the results from the grid search, we used a radial basis function as the kernel function and a gamma value of one. In Figure 5-2, Figure 5-3, and Figure 5-4, we show how changing the values of $\mathrm{C}$ and $\varepsilon$ affects the model performance by plotting the predictions made by the model on the test set along with the actual throughput values. The results are summarized in Table 1. From the table, one can see how the value of $\mathrm{C}$ strongly impacts the performance of the model.

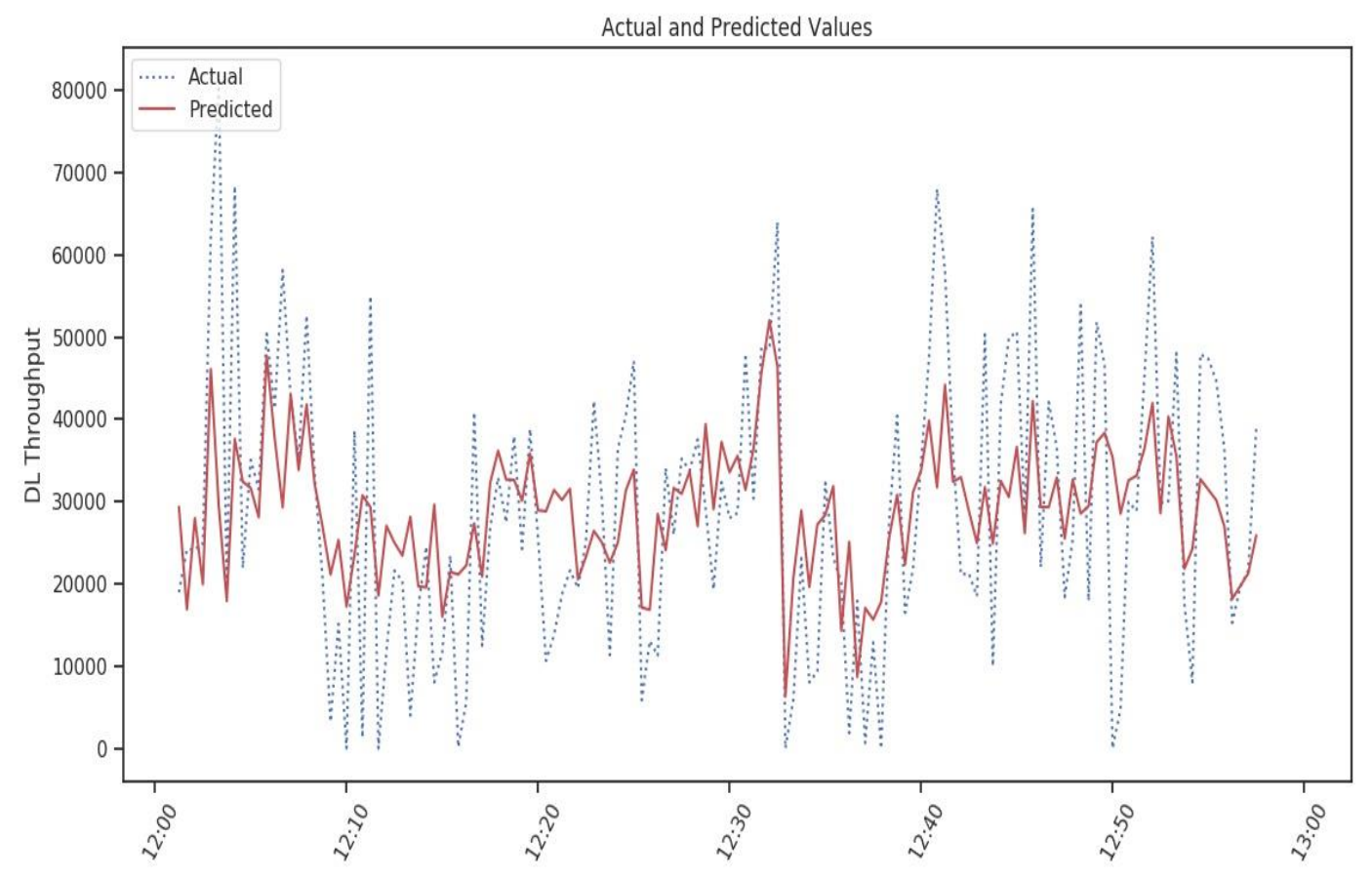

Figure 5-2: SVR model predictions with $C=10000, \varepsilon=0.01$. 


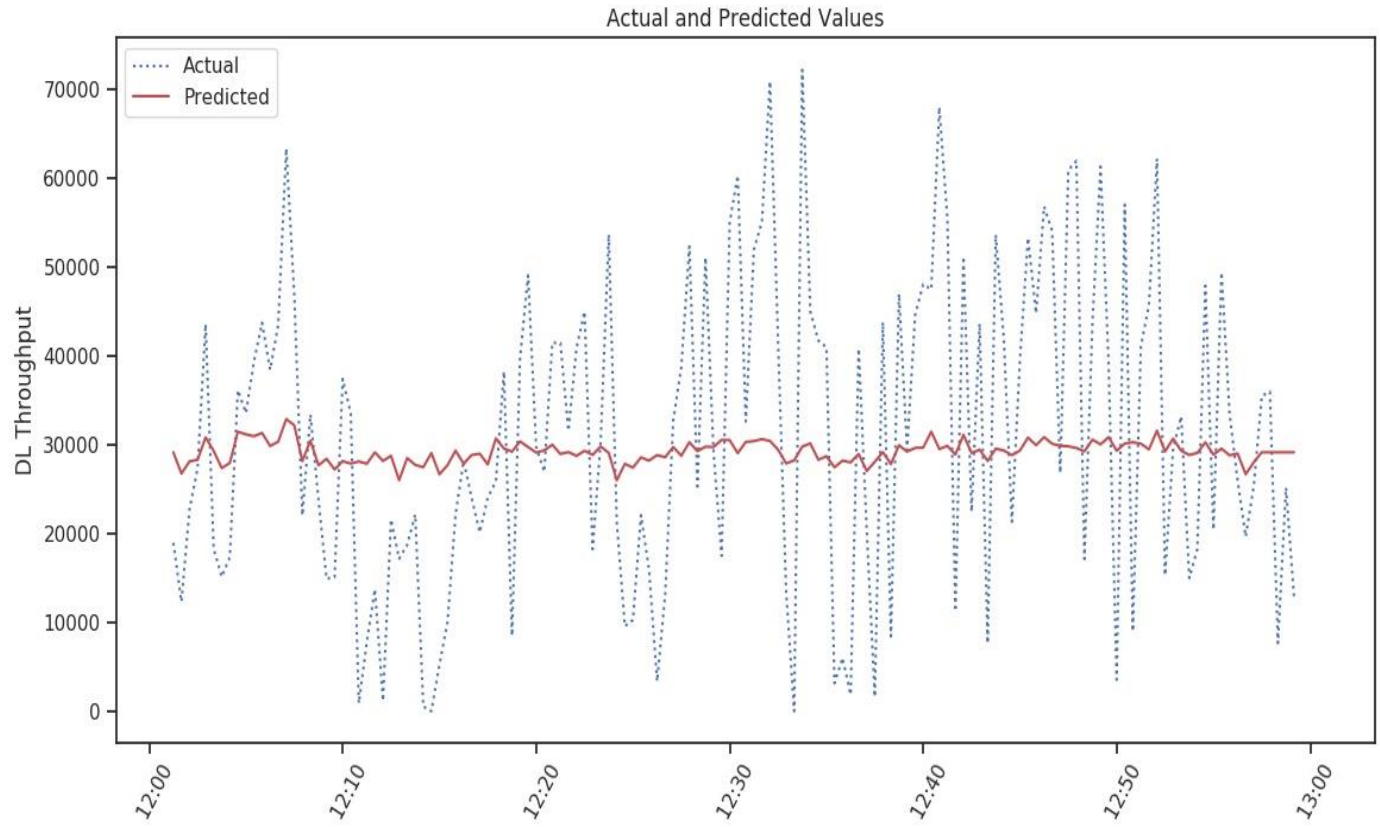

Figure 5-3: $S V R$ model predictions with $C=1000, \varepsilon=0.01$.

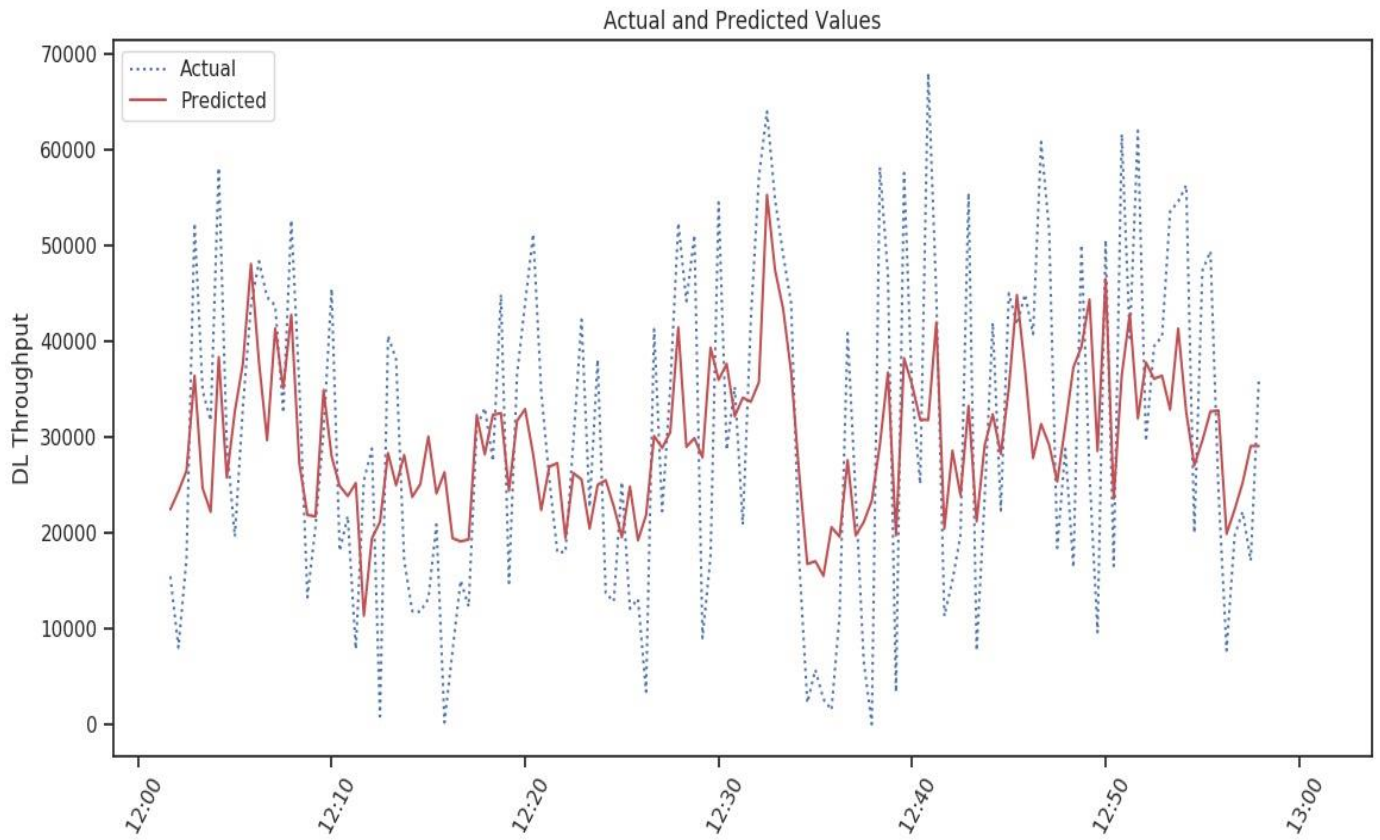

Figure 5-4: SVR model predictions with $\mathrm{C}=10000, \varepsilon=0.001$. 


\begin{tabular}{|c|c|c|}
\hline Hyperparameters & RMSE & $R^{2}$ \\
\hline $\mathrm{C}=10000, \varepsilon=0.01$ & 14000 & 0.36 \\
\hline $\mathrm{C}=1000, \varepsilon=0.001$ & 14500 & 0.30 \\
\hline $\mathrm{C}=1000, \varepsilon=0.01$ & 17000 & 0.06 \\
\hline
\end{tabular}

Table 1: SVR model evaluation using different hyperparameters

\subsubsection{Regression using KNN}

For the KNN algorithm, we trained the model using multiple $\mathrm{k}$ values (the number of neighbors) to determine the hyperparameter value that leads to the best performance. Figure 5-5, Figure 5-6, and Figure 5-7 display the model predictions as well as the actual throughput measurements. Increasing the value of $\mathrm{k}$ decreases the effect of noise and improves performance, but only to a certain limit before the error starts increasing. The results of using different $\mathrm{k}$ values are illustrated in Table 2. Based on the results shown in the Table 2, we concluded that 13 is the optimal value for the hyperparameter $\mathrm{k}$ in our problem.

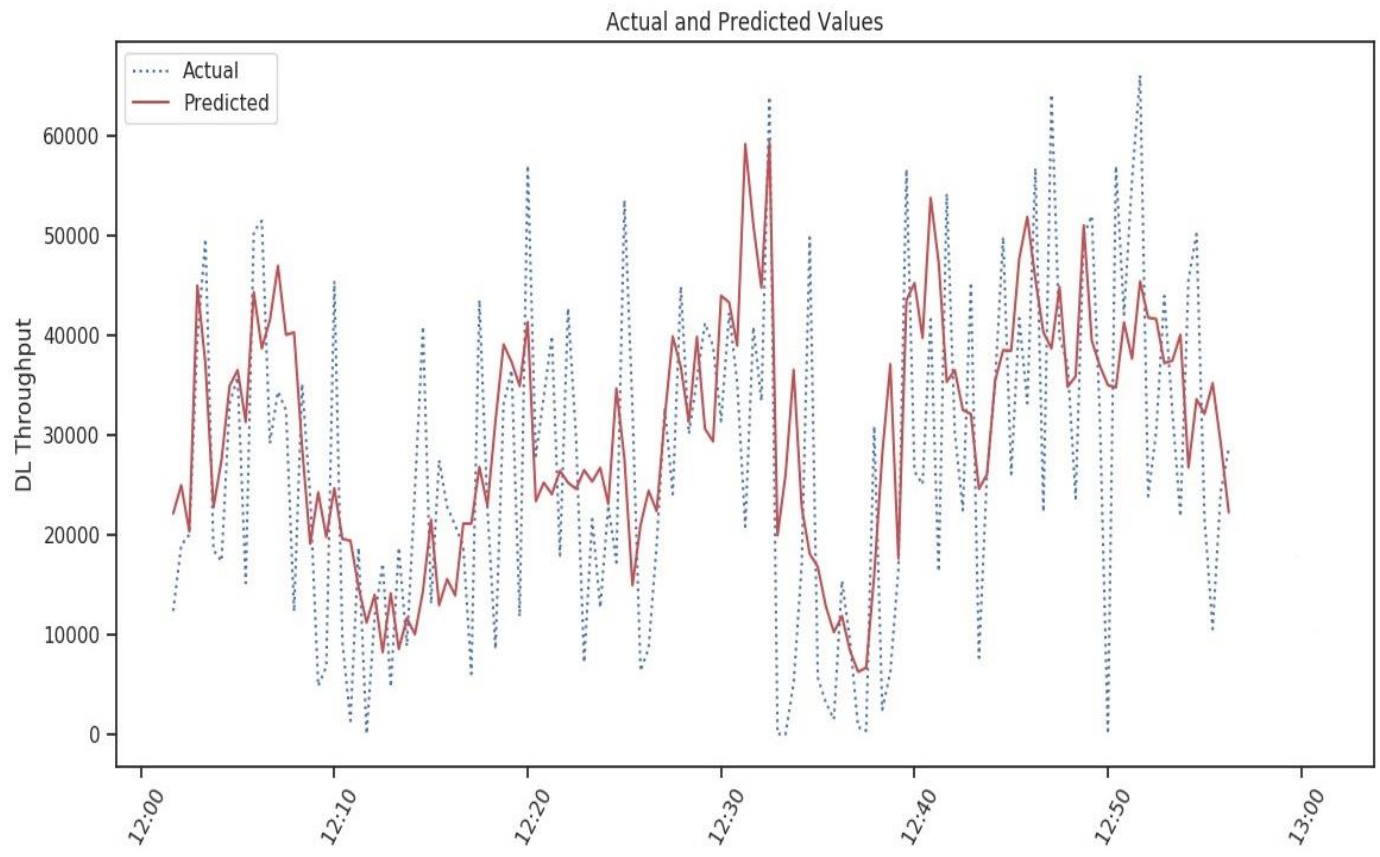

Figure 5-5: $\mathrm{KNN}$ model predictions with $\mathrm{k}=5$. 


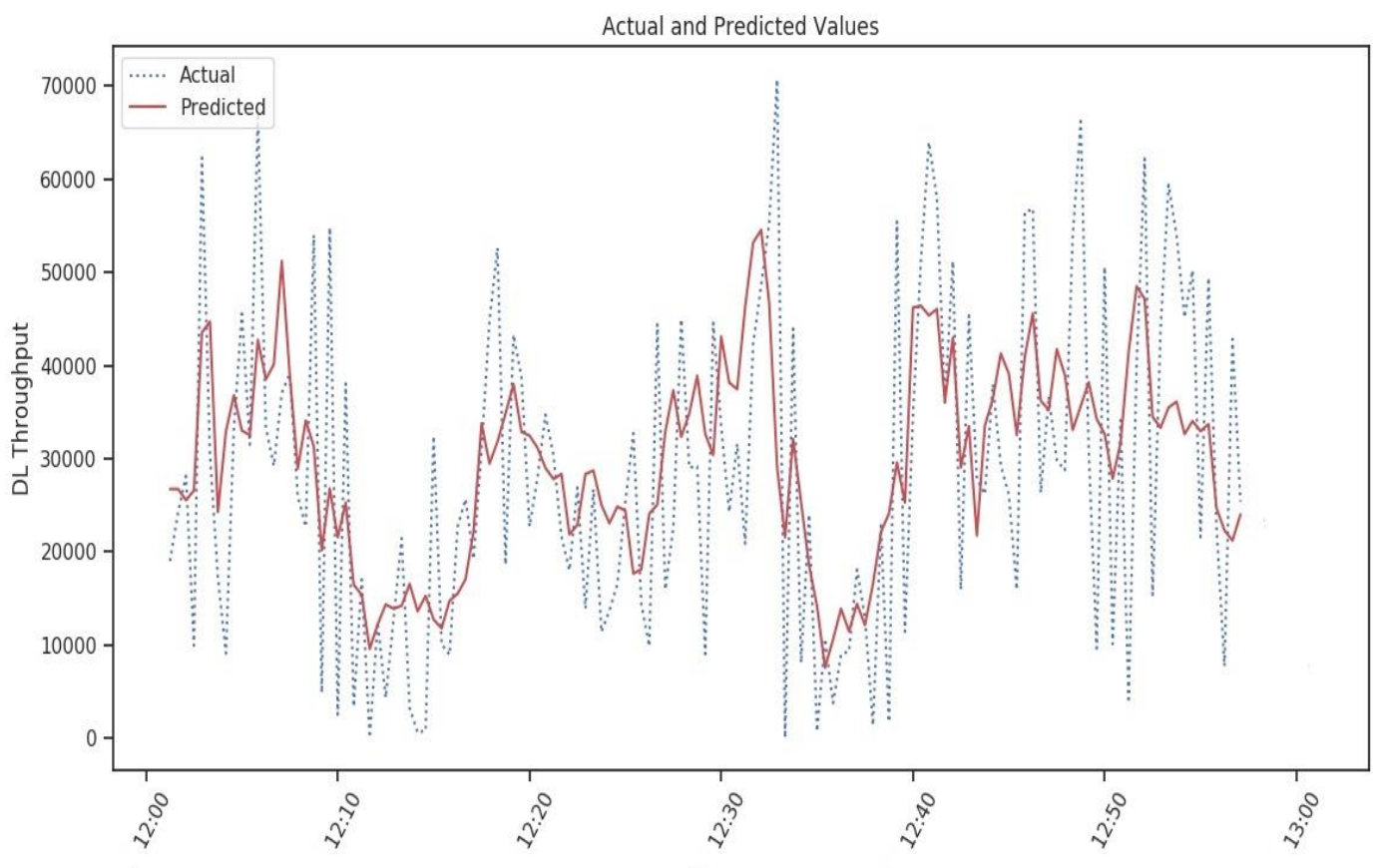

Figure 5-6: KNN model predictions with $\mathrm{k}=13$.

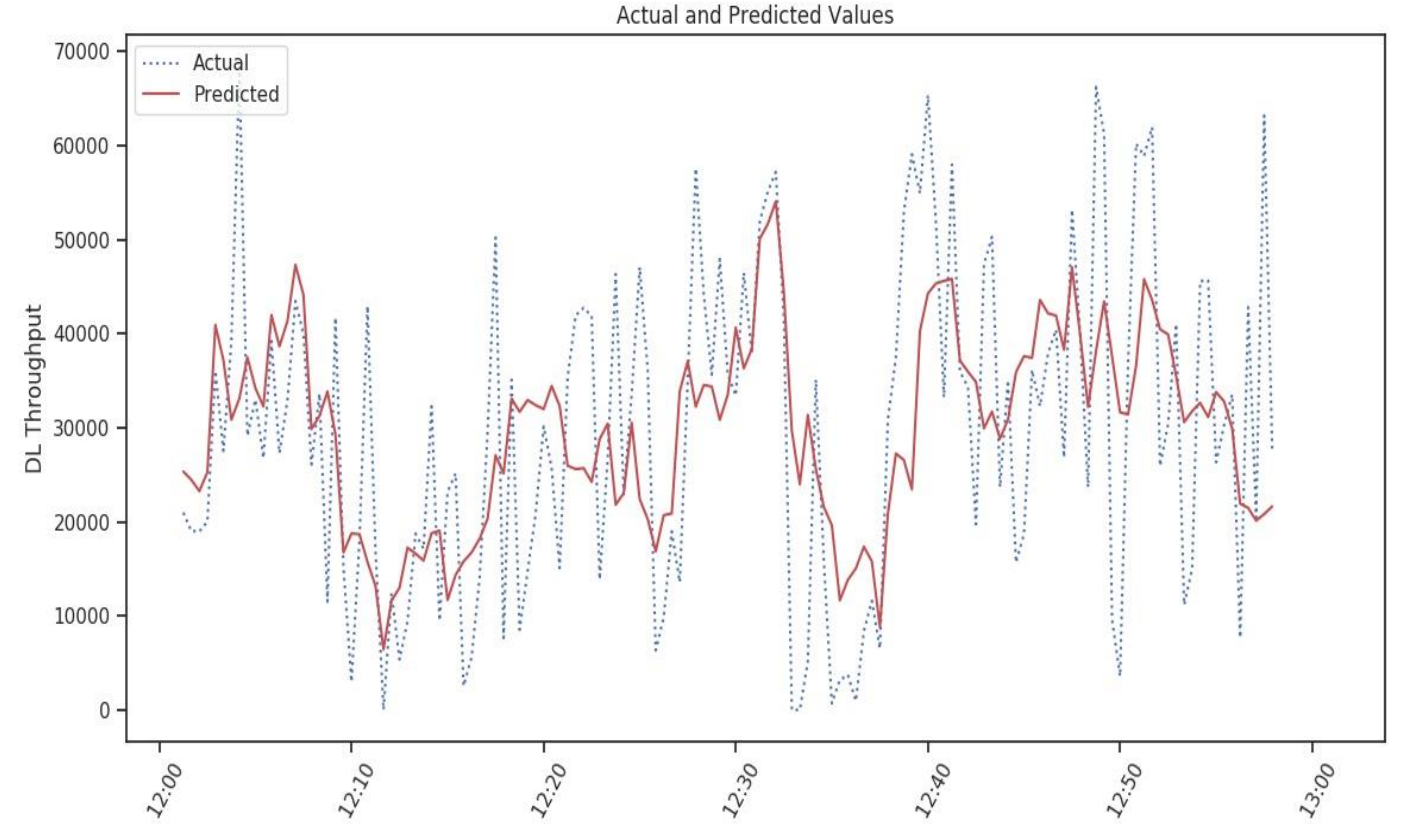

Figure 5-7: KNN model predictions with $k=17$. 


\begin{tabular}{|c|c|c|}
\hline K & RMSE & $R^{2}$ \\
\hline 5 & 15000 & 0.20 \\
\hline 13 & 13700 & 0.39 \\
\hline 17 & 14500 & 0.30 \\
\hline
\end{tabular}

Table 2: KNN model evaluation using different hyperparameters

\subsubsection{Ridge Regression}

While training the ridge regression model, we applied different values for the hyperparameter $\alpha$ (the regularization strength). To illustrate how the use of different hyperparameters affects the model performance, we present figures showing the model performance for each hyperparameter used. Figure 5-8, Figure 5-9, and Figure 5-10 show the model predictions plotted on top of the actual throughput measurements. Table 3 shows the model evaluation using different values of $\alpha$. Based on the results in the table, we concluded that a value of 10 was the optimal choice for the hyperparameter $\alpha$, as it led to the smallest prediction error on our test data.

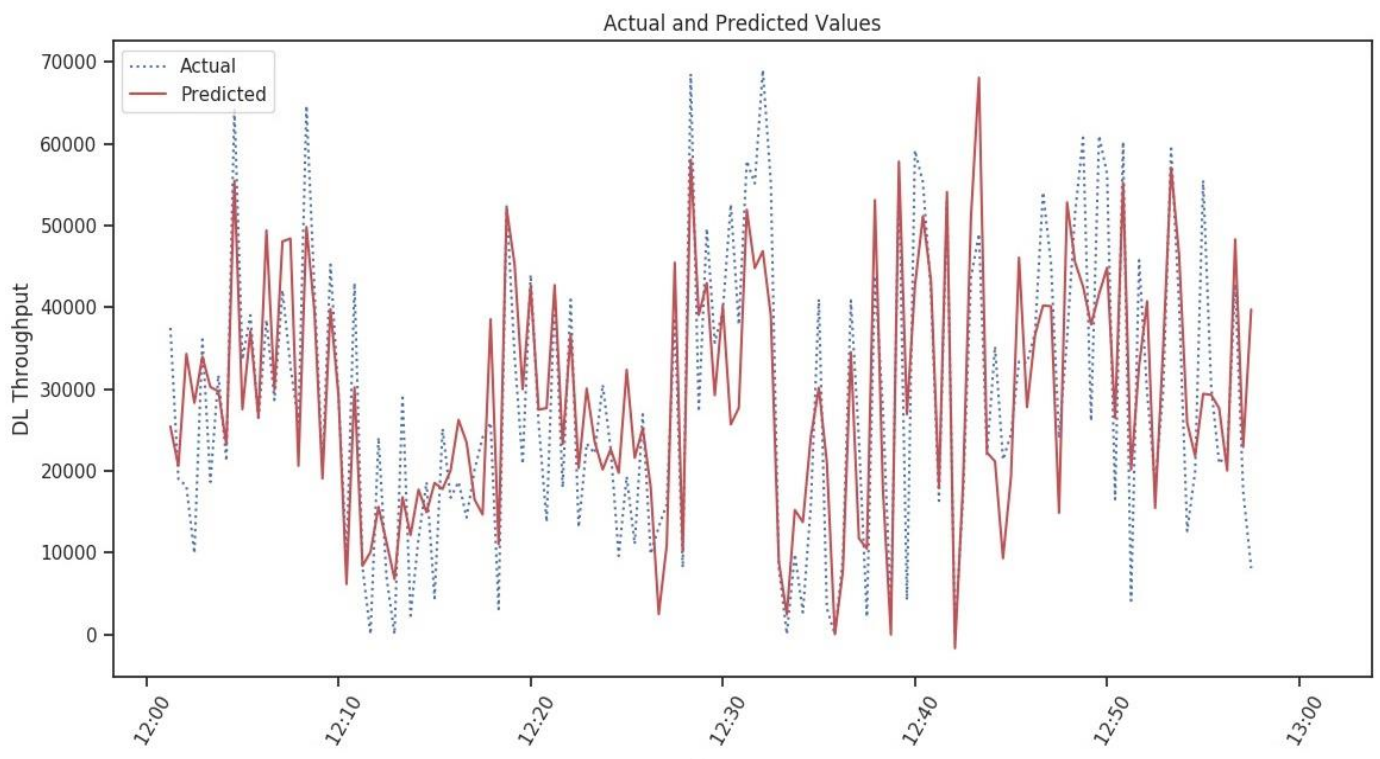

Figure 5-8: Ridge regression model predictions with $\alpha=0.1$. 


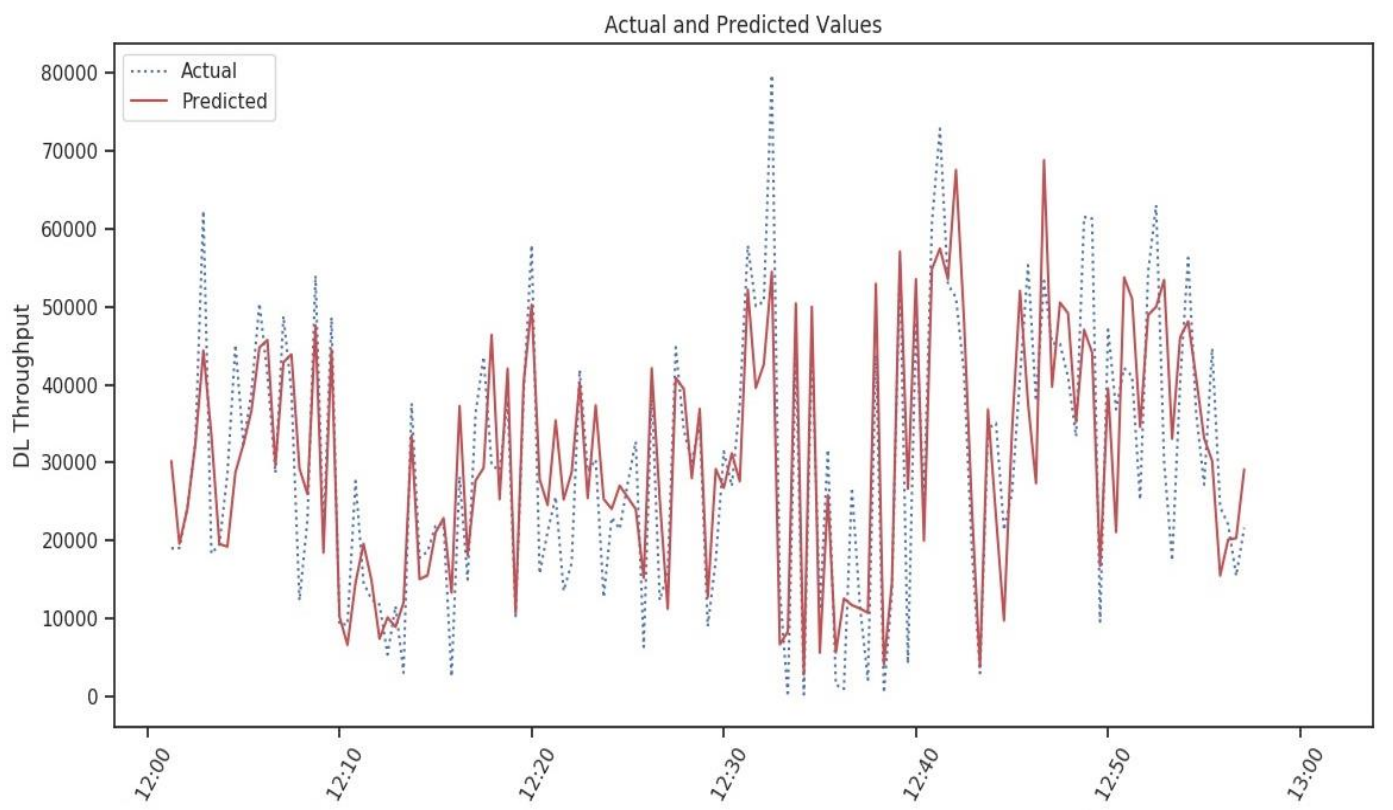

Figure 5-9: Ridge regression model predictions with $\alpha=1.5$.

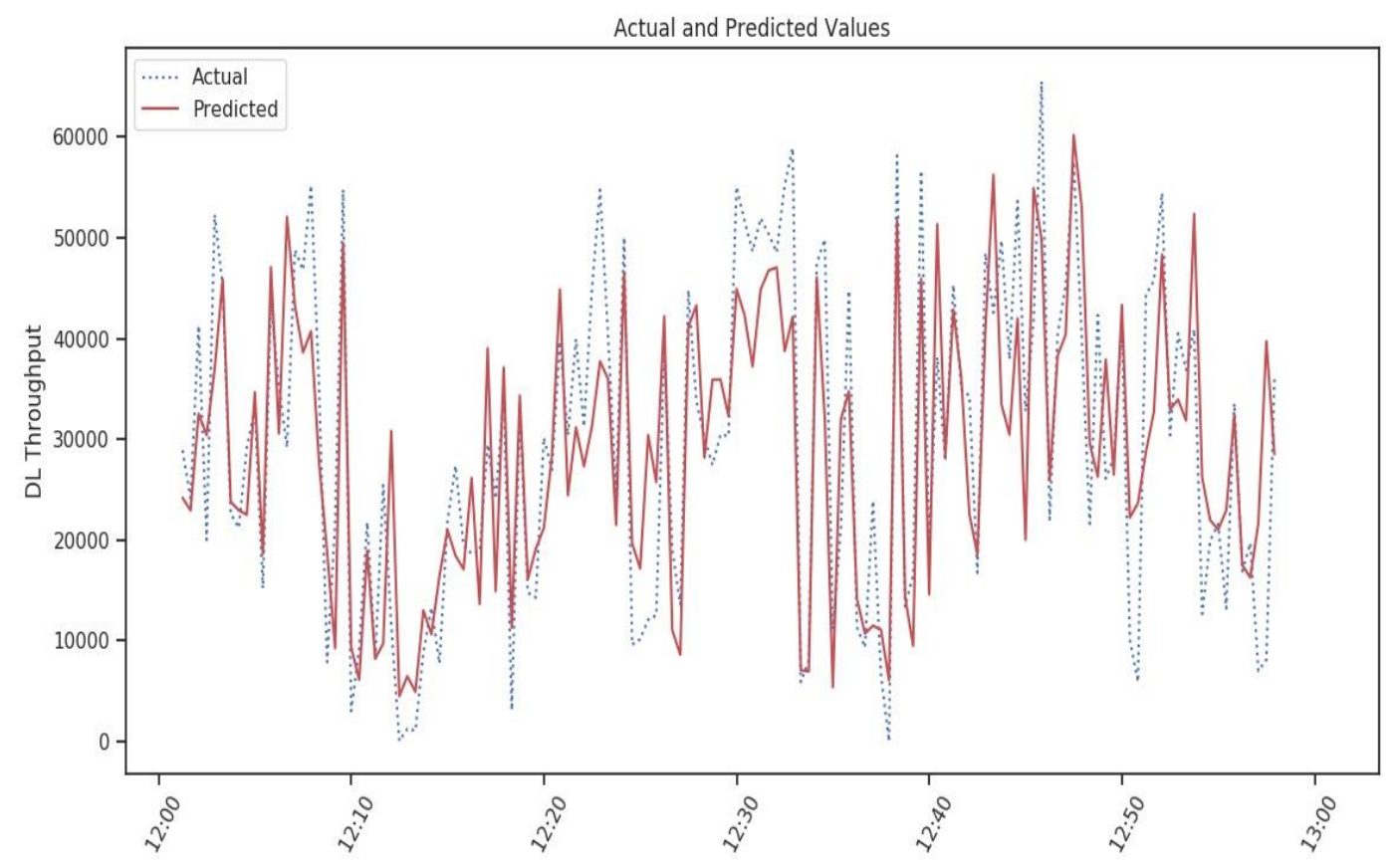

Figure 5-10: Ridge regression model predictions with $\alpha=10$. 


\begin{tabular}{|c|c|c|}
\hline$\alpha$ & RMSE & $R^{2}$ \\
\hline 0.1 & 9500 & 0.68 \\
\hline 1.5 & 9200 & 0.71 \\
\hline 10 & 9700 & 0.69 \\
\hline
\end{tabular}

Table 3: Ridge regression model evaluation using different hyperparameters

\subsubsection{Random Forest for Regression}

The performance of the random forest algorithm depends on the hyperparameter used in training, which is the number of trees. Increasing the number of trees increases the performance of the model but causes the model to have a longer training time. As with the other models, we experimented with different hyperparameters during our model training. Figure 5-11, Figure 5-12, and Figure 5-13 show the model performance with different numbers of trees. The results of using different number of trees are summarized in Table 4. Accordingly, we concluded that 400 trees were the optimal hyperparameter choice for our model.

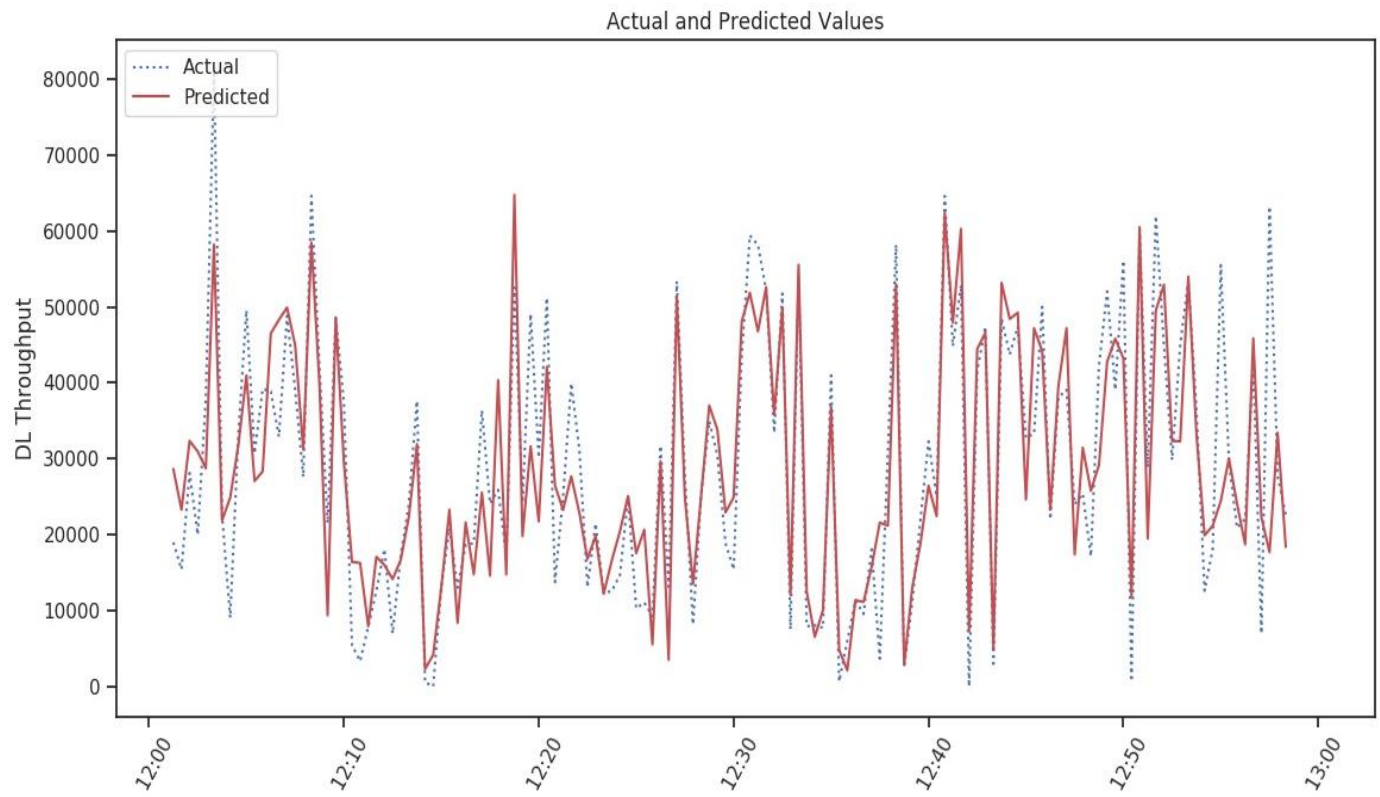

Figure 5-11: Random forest model predictions with number of trees=250. 


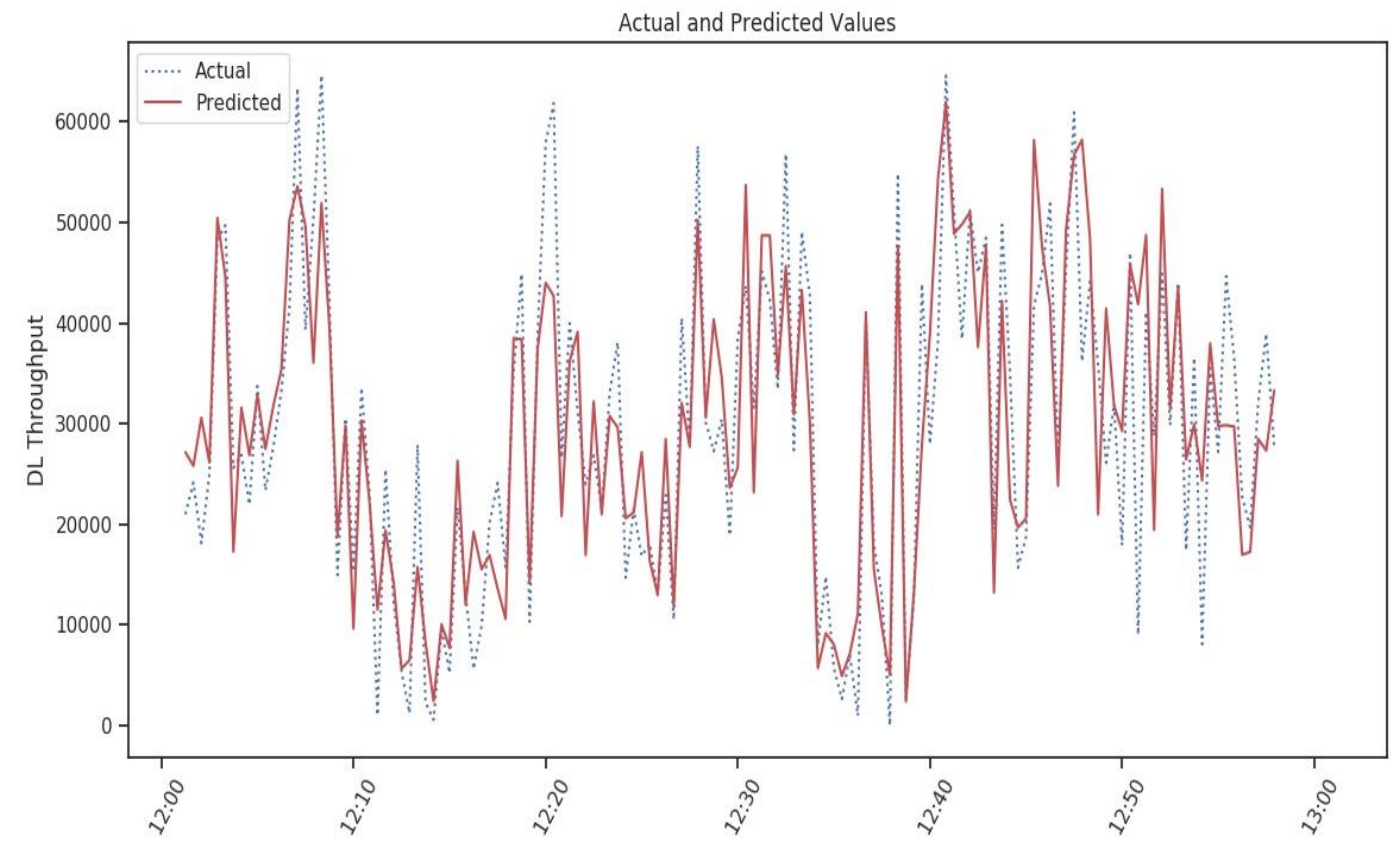

Figure 5-12: Random forest model predictions with number of trees=400.

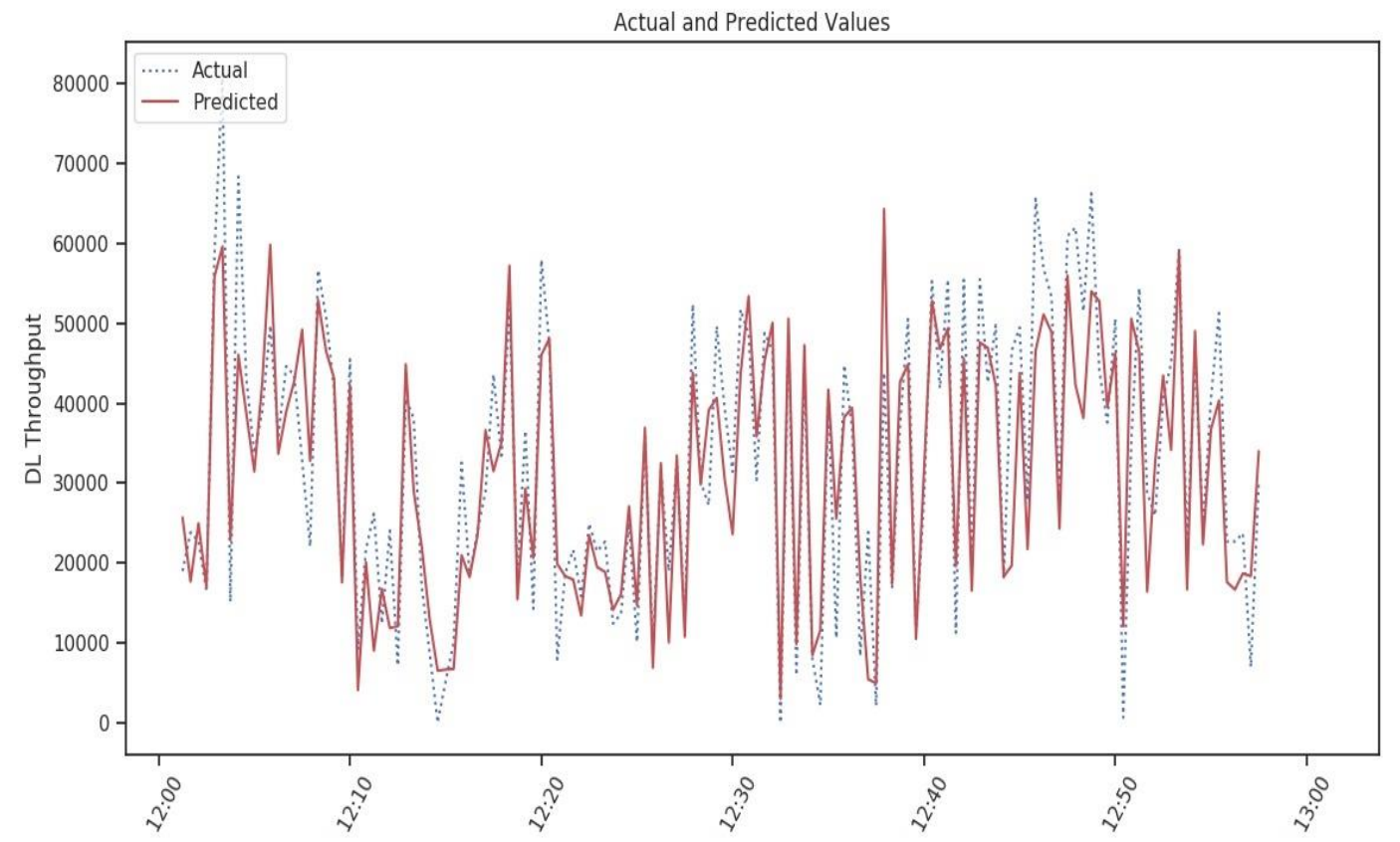

Figure 5-13: Random forest model predictions with number of trees=700. 


\begin{tabular}{|c|c|c|}
\hline Number of trees & RMSE & $R^{2}$ \\
\hline 250 & 8700 & 0.74 \\
\hline 400 & 8200 & 0.78 \\
\hline 700 & 8500 & 0.75 \\
\hline
\end{tabular}

Table 4: Random forest model evaluation using different hyperparameters

\subsubsection{Model Comparison}

To compare between the different models, we evaluated their performance using the two evaluation metrics mentioned earlier, the $R^{2}$ score and the RMSE. Table 5 shows the $R^{2}$ score and the RMSE value of each model.

\begin{tabular}{|c|c|c|}
\hline Model & $R^{2}$ & RMSE \\
\hline SVR & $0.36 \pm \mathbf{0 . 0 3}$ & $14000 \pm 300$ \\
\hline KNN & $0.38 \pm 0.02$ & $13700 \pm 300$ \\
\hline Ridge Regression & $\mathbf{0 . 7 1} \pm \mathbf{0 . 0 2}$ & $\mathbf{9 3 0 0} \pm \mathbf{2 0 0}$ \\
\hline Random Forest for Regression & $\mathbf{0 . 7 8} \pm \mathbf{0 . 0 1}$ & $\mathbf{8 2 0 0} \pm \mathbf{2 0 0}$ \\
\hline
\end{tabular}

Table 5: Throughput prediction model comparison

As shown in the table, the random forest achieved the best performance with the highest $R^{2}$ score of around 0.78 and the least RMSE value of approximately 8200 . Moreover, the performance of the ridge regression model was close to that of the random forest, with an $R^{2}$ of around 0.71 and RMSE value of around 9300. On the other hand, the performances of the KNN and the SVR models were not satisfactory. The KNN model had an $R^{2}$ score of around 0.38 and RMSE value of around 13700, while the SVR model had the lowest performance, with an $R^{2}$ score of around 0.36 and RMSE value of around 14000. 


\subsection{Results of Time Series Forecasting Models}

Along with regression predictive modelling, we applied time series forecasting techniques. We used two common approaches for time series forecasting, namely ARIMA and LSTM models. In the following subsections, we describe the models we trained along the hyperparameters tuning we performed.

\subsubsection{ARIMA Model}

As we mentioned in Chapter 4, ARIMA models have three parameters: $p, d$ and $q$, where $p$ is the number of lag observations, $\mathrm{d}$ is the degree of differencing, and $\mathrm{q}$ is the order of the moving average. The first step in determining the optimal set of parameters, is plotting the data. In Figure 5-14, one can see that our throughput time series is stationary, so differencing is not needed in the model. To find the best number of lag observations, we plotted the autocorrelation graph of our time series in Figure 5-15. From the figure, a strong positive correlation in the first five lags can be seen, so we chose a p parameter value of five. Moreover, after trying various q values, we reached the highest performance with a value of five. Figure 5-16 shows the results of using an ARIMA model on our dataset for forecasting the throughput values. The evaluation of this model displayed an RMSE value of 10400 and an $R^{2}$ score of 0.62 on the testing data.

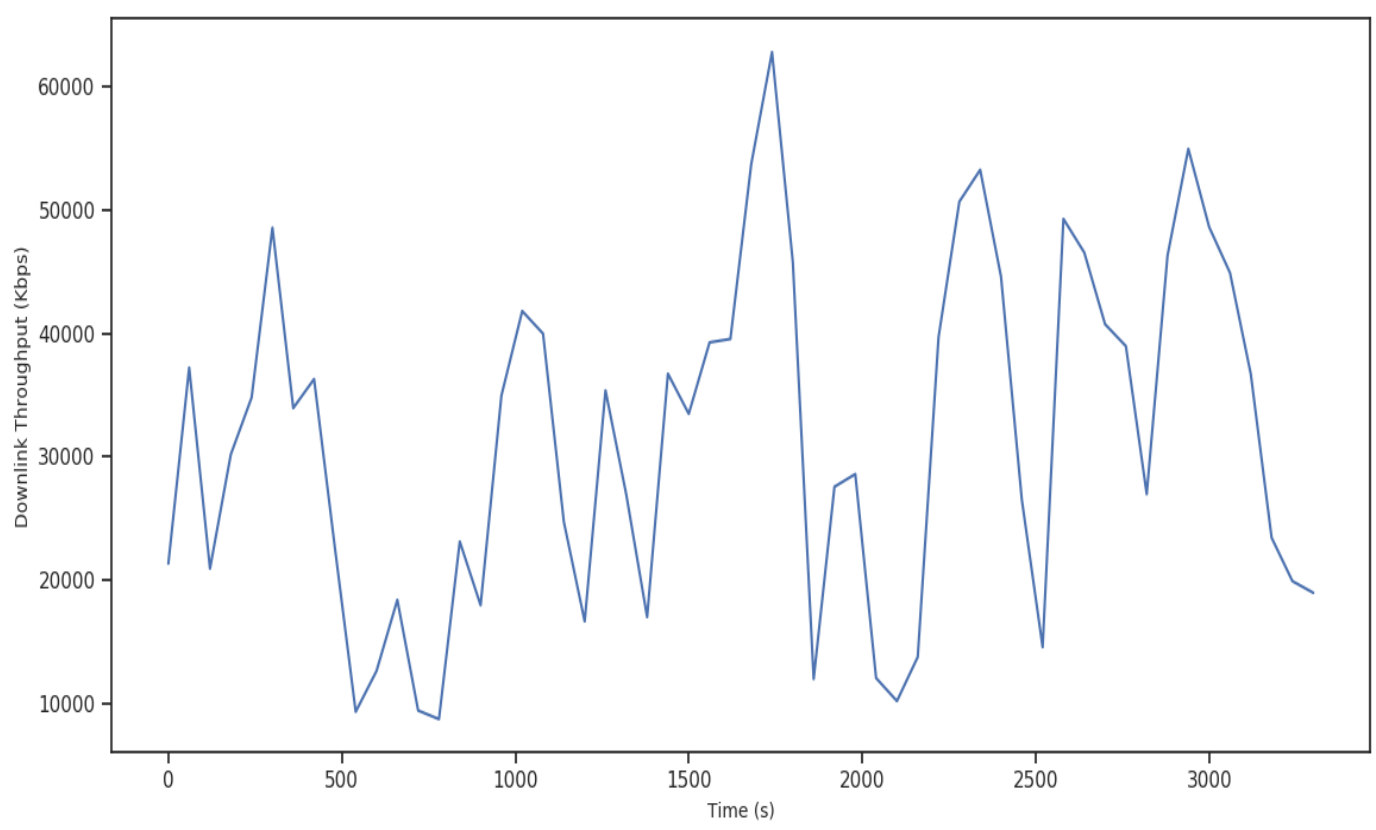

Figure 5-14: Throughput vs. Time. 


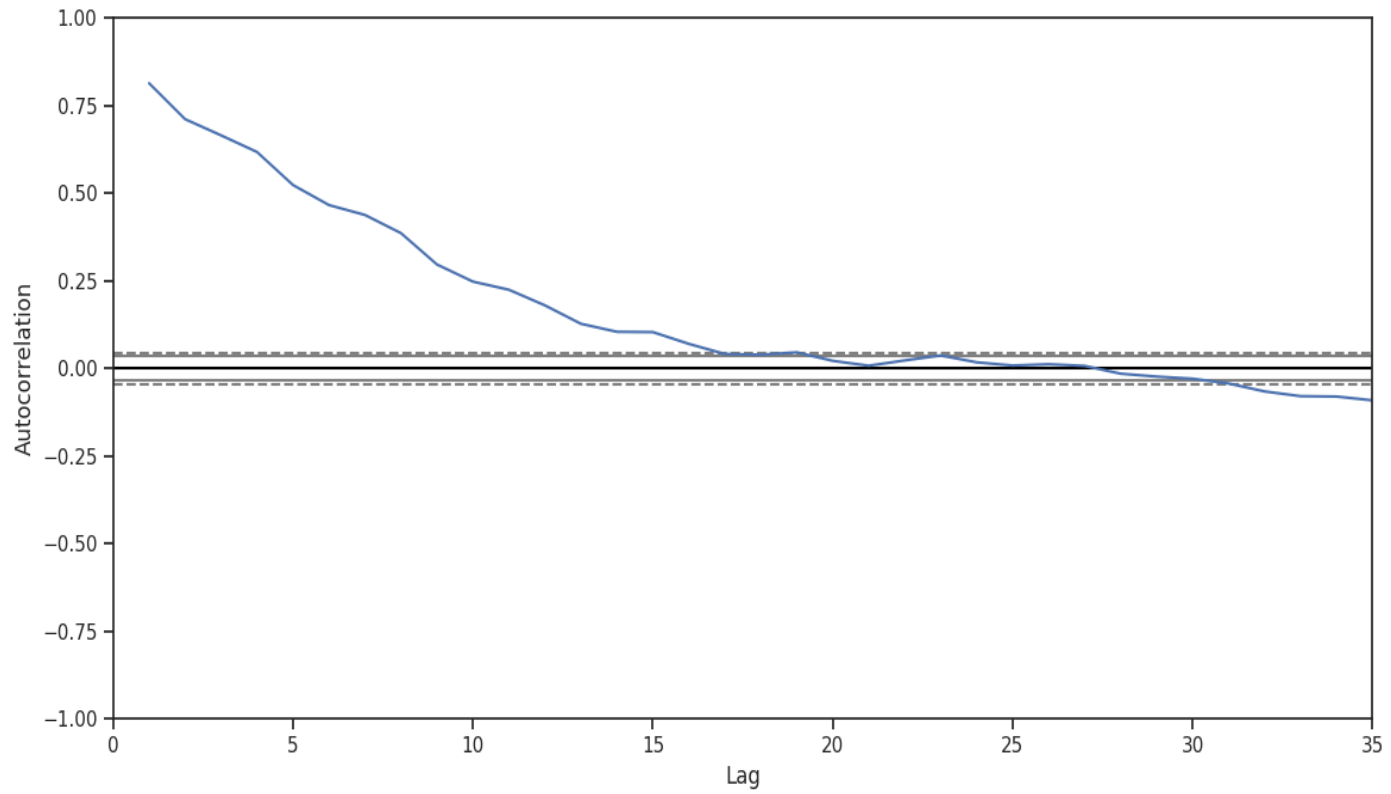

Figure 5-15: Autocorrelation plot of the time series.

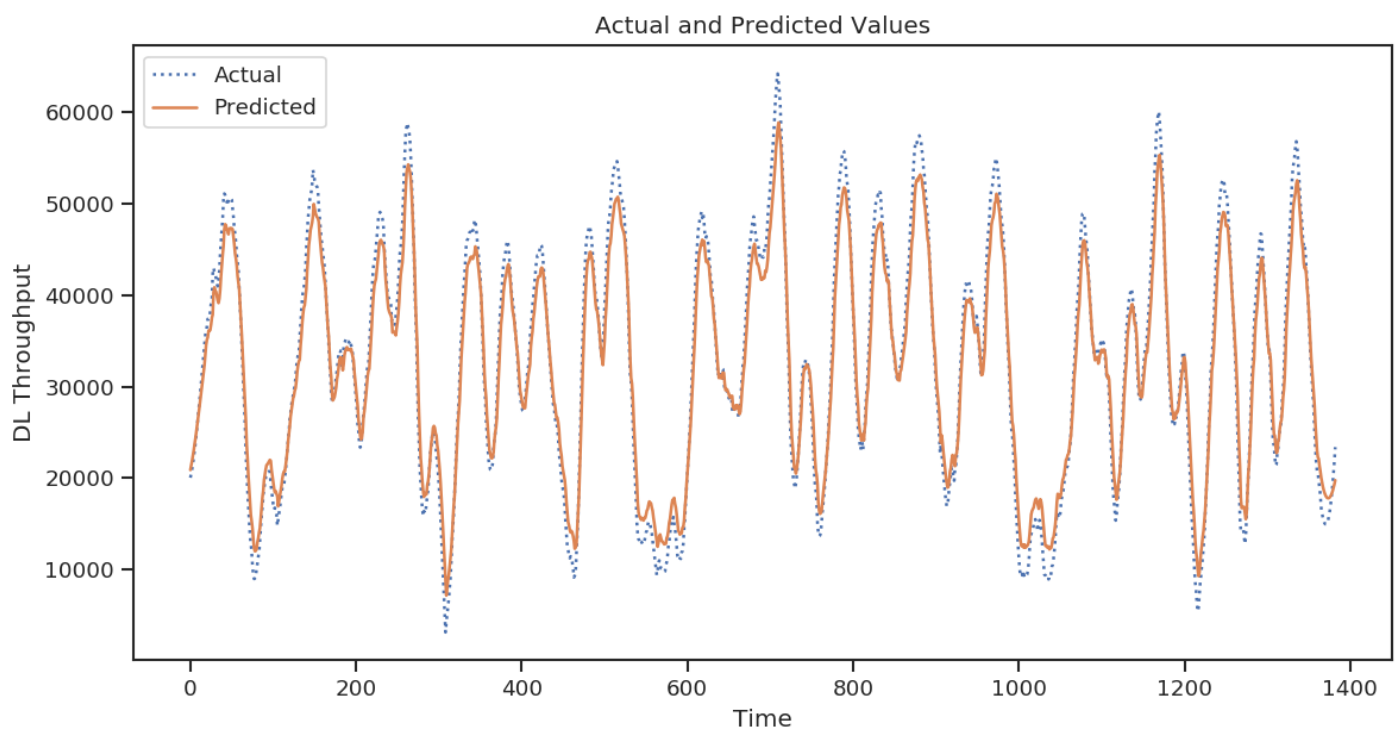

Figure 5-16: ARIMA model performance for time series throughput forecasting. 


\subsubsection{LSTM Model}

For the LSTM model, there are multiple hyperparameters to be tuned, such as the number of layers in the network, the learning rate, and the number of epochs. Furthermore, additional features such as a dropout layer could be added to the model to prevent overfitting. To reach a high level of model performance, we should select a dropout value that prevents overfitting while still retaining the model accuracy. We experimented with different hyperparameters to reach the highest performance possible. Figure 5-17 shows the architecture of the LSTM model that we used for throughput forecasting. We used two LSTM layers and one fully connected layer, a learning rate of $0.01,100$ epochs, and a dropout of 20\%. Figure 5-18 displays the model performance on our time series data for throughput forecasting. The model shown in the figure had a RMSE value of 10800 and an $R^{2}$ score of 0.59 on the testing data.

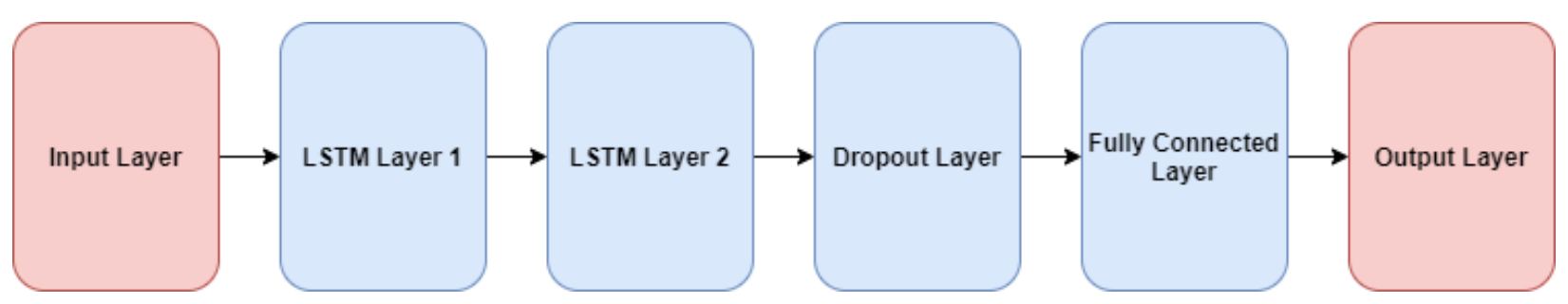

Figure 5-17: LSTM model architecture.

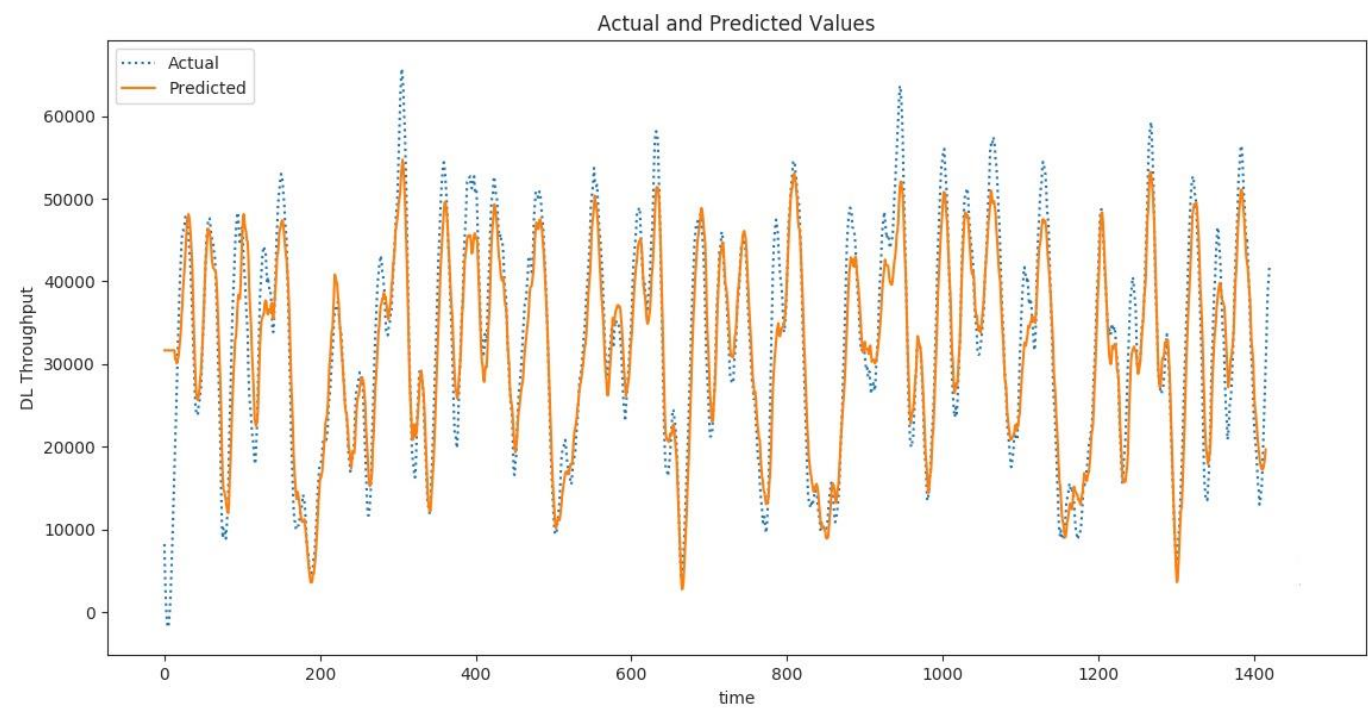

Figure 5-18: LSTM model performance for time series throughput forecasting. 


\subsection{Discussion}

As we have seen in Table 5, the random forest model had the highest throughput prediction performance on our dataset. The reason for this high performance is that random forests have a strong generalization edge that prevents overfitting and improves the accuracy of the model. By choosing a few random subsamples to build each tree and selecting a random set of features, the random forest algorithm decreases the correlation between the different trees and greatly reduces the variance in the predictions made by the model.

For the time series forecasting, the ARIMA model had a slightly higher performance than the LSTM model as the LSTM networks require a large amount of data. This is mainly because LSTMs have various units that require weights to be trained.

We believe that we could achieve higher throughput prediction performance if we had access to additional data from network operators as in the work of [29]. Training the models on data from network operators along with our client-side data would significantly improve the prediction performance.

In addition, having a dataset with a higher granularity as in the related work in [7] would improve the accuracy of the data and therefore lead to a higher prediction performance.

\subsection{Summary}

In this chapter, we have described how we collected and prepared the data for throughput prediction algorithms. Furthermore, we explained how we experimented with different hyperparameters during the training of the machine learning models for throughput prediction. Moreover, we evaluated the performance of each model and performed a comparison between the different models in order to determine the optimal one for our problem. Additionally, we presented the results of applying time series forecasting algorithms on our data. Lastly, we discussed the results that we achieved and explained how we could further improve the prediction performance. 


\section{Chapter 6}

\section{Conclusions and Future Directions}

The advancements in the cellular network technologies over the past decade have brought endless services and capabilities to the users. Smartphone users today rely on their phones for work as well as leisure activities, such as online gaming and video streaming. This has increased the load on cellular networks, causing the network traffic to increase. Cellular network operators are always looking for solutions to cope with this rising demand by developing new mechanisms for resource allocation and load balancing. One way to improve the network QoS is to predict the fluctuations in the network connectivity before they occur, in order to take preventative actions. Accordingly, several researchers have proposed the concept of throughput analysis as a method for determining the network quality in advance. Such an analysis requires substantial amounts of network data. However, there is a noticeable deficiency in the 4G LTE network data in the research community.

In this work, we executed a measurement study along routes taken by public transportation buses in Kington, Ontario, collecting data during 30 different bus trips. We collected measurements of various 4G LTE network parameters, downlink and uplink throughput, and context information such as GPS locations and speed. To account for changes in the road traffic and network connectivity levels, we conducted the measurements at three different times of the weekday: 9 am, $12 \mathrm{pm}$ and $6 \mathrm{pm}$. Additionally, we performed an in-depth analysis of the collected data to investigate the effects of the geographical area, time and contextual factors such as bus stops and number of passengers on the network performance and quality. To be able to anticipate the fluctuations in the network connectivity and enhance the QoS, we applied throughput prediction techniques on our data. In particular, we performed multiple preprocessing steps on our data and then trained and tested various machine learning algorithms for throughput prediction, namely SVR, KNN, ridge regression, and random forest. Moreover, we deployed time series forecasting models on the collected data for throughput prediction. We then compared the results of the different models deployed 
by using different evaluation metrics in order to determine the optimal one for throughput prediction based on our data.

Our analysis shows that there are significant fluctuations in the network connectivity at different times of the day. At $6 \mathrm{pm}$, the mean signal strength and throughput measurements are notably less than at 9 am and $12 \mathrm{pm}$. Furthermore, the location variations at $6 \mathrm{pm}$ were much higher than at the other two times of the day. We believe this occur because there is more traffic on the road and more passengers on the bus at 6 pm, which in turn causes an increased network traffic at this time. From these observations we conclude that the time of the day and the road traffic condition play an important role in controlling the network QoS. Moreover, we showed that some locations along the bus route experience remarkably lower signal strength and throughput values. This is mainly due to the lack of cell towers located in these areas, compared to other areas along the bus route. We feel that network operators could greatly enhance the network connectivity by deploying more towers at these locations.

For throughput prediction, we achieved the highest prediction accuracy using the random forest algorithm. This result is mostly due to the fact that random forests add an additional layer of randomness to the features, which greatly reduces the variance and causes the model to have a strong generalization power. Moreover, the additional layer of randomness, added by the random forests, leads to a higher model performance on unseen data and prevents overfitting.

\section{Future Directions}

The solution presented in this thesis can be further improved. In the following points, we suggest future directions to research that could improve our solution, as well as further studies that could be conducted on our dataset.

1. By collecting more measurements of cellular network parameters, the performance of the prediction models can be greatly enhanced.

2. Acquiring additional data from network operators, such as average cell throughput and average number of users per cell, could significantly benefit the throughput prediction models. 
3. User movement prediction could be performed by investigating the correlation between mobility and cellular network parameters.

4. Since the dataset contains information about the GPS locations of the device and serving cells and channel parameters of the serving cell and neighboring cells, handover analysis could be applied on the data.

5. The data could be used for predictive resource allocation mechanisms, such as content prefetching, which rely on the user location awareness. 


\section{References}

[1] Q. Xu, S. Mehrotra, Z. Mao, and J. Li, "PROTEUS: network performance forecast for real-time, interactive mobile applications," in Proc. of the 11th Annual International Conf. on Mobile Systems, Applications, and Services, pp. 347-360, 2013.

[2] H. Abou-zeid, H. S. Hassanein, Z. Tanveer and N. AbuAli, "Evaluating mobile signal and location predictability along public transportation routes," IEEE Wireless Communications and Networking Conference (WCNC), New Orleans, LA, pp. 1195-1200, 2015.

[3] R. Margolies, A. Sridharan, V. Aggarwal, R. Jana, N. K. Shankaranarayanan, V. A. Vaishampayan, and G. Zussman, "Exploiting mobility in proportional fair cellular scheduling: Measurements and algorithms," in Proc. IEEE INFOCOM, pp. 1339-1347, 2014.

[4] D. Zhou, Z. Yan, Y. Fu, and Z. Yao, “A survey on network data collection”, Journal of Network and Computer Applications, Journal of Network and Computer Applications, vol. 116, 15, pp. 9$23,2018$.

[5] K. Connolly, Law of Internet Security and Privacy. Aspen Publishers, 2001, pp. 131-132.

[6] Qualcomm, Qualcomm eXtensible Diagnostic Monitor (QXDM Professional), 2008. [Online]. Available: http://www.qualcomm.com/media/documents/tags/qxdm. [Accessed: Jan. 25, 2020].

[7] R. Jin, Enhancing upper-level performance from below: Performance measurement and optimization in LTE networks. Doctoral Dissertations, Univ. of Connecticut, United States, 2015.

[8] Wikipedia contributors, "Crowdsourcing”. Wikipedia, The Free Encyclopedia. [Online]. Available: https://en.wikipedia.org. [Accessed: Feb. 9, 2020].

[9] F. Afroz, R. Subramanian, R. Heidary, K. Sandrasegaran, and S. Ahmed, "SINR, RSRP, RSSI and RSRQ Measurements in Long Term Evolution Networks," International Journal of Wireless \& Mobile Networks, pp. 113-123, 2015.

[10] G. Miao, J. Zander, K. Sung, and B. Slimane, "Fundamentals of Mobile Data Networks," Cambridge University Press, 2016. 
[11] M. Sauter, From GSM to LTE: An Introduction to Mobile Networks and Mobile Broadband, 1st ed., Wiley Publishing, 2011.

[12] S. Farahani, ZigBee Wireless Networks and Transceivers, 2008, pp. 225-246.

[13] M. Posinna. "Different types of fiber optic cables". HFCL, 2014.

[14] C. M. Bishop, Pattern Recognition and Machine Learning, New York: Springer, 2006.

[15] S. J. Russell and P. Norvig, Artificial Intelligence: A Modern Approach, 3rd Edition, Prentice Hall, 1995.

[16] E. Alpaydin, "Introduction to Machine Learning," MIT Press, p. 9, 2010.

[17] R. D. Cook and S. Weisberg, "Criticism and Influence Analysis in Regression," Sociological Methodology, vol. 13. pp. 313-361, 1982.

[18] G. Hinton and T. Sejnowski, "Unsupervised Learning: Foundations of Neural Computation,” MIT Press, 1999.

[19] R. C. Tryon. Cluster Analysis: Correlation Profile and Orthometric (factor) Analysis for the Isolation of Unities in Mind and Personality, Edwards Brothers, 1939.

[20] S. T. Roweis and L. K. Saul, "Nonlinear Dimensionality Reduction by Locally Linear Embedding," Science, vol. 290 pp. 2323-2326, 2000.

[21] L. P. Kaelbling, M. L. Littman, and A. W. Moore, "Reinforcement Learning: A Survey," Journal of Artificial Intelligence Research, vol. 4, pp. 237-285, 1996.

[22] X. K. Zou, J. Erman, V. Gopalakrishnan, E. Halepovic, R. Jana, X. Jin, J. Rexford, and R. K. Sinha. "Can Accurate Predictions Improve Video Streaming in Cellular Networks?" In Proc. of the 16th International Workshop on Mobile Computing Systems and Applications (HotMobile '15), association for Computing Machinery, New York, USA, pp. 57-62, 2015.

[23] F. Jomrich, A. Herzberger, T. Meuser, B. Richerzhagen, R. Steinmetz, and C. Wille, "Cellular Bandwidth Prediction for Highly Automated Driving - Evaluation of Machine Learning Approaches based on Real-World Data," in Proc. of the 4th International Conf. on Vehicle 
Technology and Intelligent Transport Systems, vol.1, pp. 121-132, Funchal, Madeira, Portugal, 2018.

[24] F. Lu, H. Du, A. Jain, G. M. Voelker, A. C. Snoeren, and A. Terzis, "CQIC: Revisiting cross-layer congestion control for cellular networks," in Proc. of the 16th International Workshop on Mobile Computing Systems and Applications, pp. 45- 50, 2015.

[25] J. Yao, S. S. Kanhere, and M. Hassan, "An empirical study of bandwidth predictability in mobile computing," in Proc. ACM WiNTECH, pp. 11-18, 2008.

[26] D. Han, J. Han, Y. Im, M. Kwak, T. T. Kwon, and Y. Choi, "MASERATI: Mobile adaptive streaming based on environmental and contextual information," in Proc. ACM WiNTECH, pp. 3340, 2013.

[27] A. Samba, Y. Busnel, A. Blanc, P. Dooze, G. Simon, "Instantaneous Throughput Prediction in Cellular Networks: Which Information Is Needed?" in IFIP/IEEE International Symposium on Integrated Network Management (IM), Lisbonne, Portugal, 2017.

[28] D. Raca, J.J. Quinlan, A.H. Zahran, C.J. Sreenan. "Beyond Throughput: a 4G LTE Dataset with Channel and Context Metrics," in Proc. of ACM Multimedia Systems Conference, Amsterdam, The Netherlands, 2018.

[29] T. Kamakaris and J. V. Nickerson, "Connectivity maps: Measurements and applications," in Proc. of the 38th Annual Hawaii International Conf. on System Sciences, Big Island, HI, USA, pp. 307$307,2005$.

[30] T. Pögel, and L. Wolf, "Prediction of 3G network characteristics for adaptive vehicular connectivity maps (poster)," in Vehicular Networking Conf. (VNC), IEEE, pp. 121-128, 2012.

[31] T. Pögel, and L. Wolf, "Optimization of vehicular applications and communication properties with connectivity maps," in Local Computer Networks Conference Workshops (LCN Workshops), IEEE 40th, pp. 870-877, 2015. 
[32] Y. Liu, and J. Y. Lee, "An empirical study of throughput prediction in mobile data networks," in Global Communications Conference (GLOBECOM), IEEE, pp. 1-6, 2015.

[33] M. Sauter, "Mobility Management in the Cell-DCH State," in GSM to LTE: An Introduction to Mobile Networks and Mobile Broadband. John Wiley \& Sons, pp. 160-160, 2010. [Online].

[34] D. Wu, Y. Li and Y. Sun, "Construction and Block Error Rate Analysis of Polar Codes Over AWGN Channel Based on Gaussian Approximation," in IEEE Communications Letters, vol. 18, no. 7, pp. 1099-1102, July 2014.

[35] M. Hazewinkel, "Normal distribution," in Encyclopedia of Mathematics, Springer Science+Business Media B.V. / Kluwer Academic Publishers, 2001.

[36] S. McKillup, Statistics explained: An Introductory guide for life scientists, Cambridge: Cambridge University Press, 2011.

[37] G. S. Maddala, "Outliers," in Introduction to Econometrics, 2nd ed. New York: MacMillan. pp. 89-90, 1992.

[38] E. Kreyszig, Advanced Engineering Mathematics, 4th ed. Wiley. pp. 880-881, 1979.

[39] G. Upton, I. Cook, “Understanding Statistics,” Oxford University Press. p. 55, 1996.

[40] M. Ester, H.-P. Kriegel, J. Sander, and X. Xu, “A Density-Based Algorithm for Discovering Clusters in Large Spatial Databases with Noise.," in KDD, pp. 226-231, 1996.

[41] F. T. Liu, K. M. Ting, Z. H. Zhou, "Isolation Forest," in 2008 Eighth IEEE International Conf. on Data Mining: 413-422, 2008.

[42] C. C. Aggarwal and S. Sathe, Outlier ensembles: An introduction. Springer. 2017.

[43] F. Pedregosa, G. Varoquaux, A. Gramfort, V. Michel, B. Thirion, O. Grisel, M. Blondel, P. Prettenhofer, R. Weiss, V. Dubourg, J. Vanderplas, A. Passos, D. Cournapeau, M. Perrot, and E. Duchesnay "Scikit-learn: Machine Learning in Python," Journal of Machine Learning Research, vol. 12, pp. 2825-2830, 2011. 
[44] N. S. Altman, "An introduction to kernel and nearest-neighbor nonparametric regression," The American Statistician, vol. 46, no.3, pp. 175-185, 1992.

[45] H. Anton, Elementary Linear Algebra, 7th ed., John Wiley \& Sons, pp. 170-171, 1994.

[46] P. E. Black, Dictionary of Algorithms and Data Structures, 2006.

[47] A. Singhal, "Modern Information Retrieval: A Brief Overview," Bulletin of the IEEE Computer Society Technical Committee on Data Engineering, vol. 24, no. 4, pp. 35--43, 2001.

[48] D. J. S. Robinson, An Introduction to Abstract Algebra. Walter de Gruyter, pp. 255-257, 2003.

[49] M. J. Azur, E. A. Stuart, C. Frangakis, P. J. Leaf, "Multiple imputation by chained equations: what is it and how does it work?," International Journal of Methods in Psychiatric Research, vol. 20, no. 1, pp. 40-49, 2011.

[50] A. Jain, K. Nandakumar, A. Ross, "Score normalization in multimodal biometric systems," Pattern Recognition, vol. 38, no. 12, pp. 2270-2285, 2005.

[51] S. Cannistra, "Small explanation of binning in image processing". [Online]. Available: http://www.starrywonders.com/binning.html. [Accessed Feb. 10, 2020].

[52] K. Pearson, "On the criterion that a given system of deviations from the probable in the case of a correlated system of variables is such that it can be reasonably supposed to have arisen from random sampling," The London, Edinburgh, and Dublin Philosophical Magazine and Journal of Science, vol. 50, no. 302, pp. 157-175, Jul. 1900.

[53] S. Stringer, "Feature Importance - What's in a name?" July, 2018, Medium. [Online]. Available: https://medium.com/bigdatarepublic/feature-importance-whats-in-a-name-79532e59eea3. [Accessed Feb. 10, 2020].

[54] T. C. Urdan, Statistics in plain English, Santa Clara University, 2016. 
[55] A. N. Tikhonov, A. Goncharsky, V. V. Stepanov, A. G. Yagola, Numerical Methods for the Solution of Ill-Posed Problems. Netherlands: Springer Netherlands, 1995.

[56] X. Yan, "Linear Regression Analysis: Theory and Computing," World Scientific, pp. 1-2, 2009.

[57] T. J. Archdeacon, "Correlation and regression analysis: a historian's guide," University of Wisconsin Press. pp. 161-162, 1994.

[58] W.M.P. Van der Aalst, V. Rubin, H.M.W. Verbeek, B. F. Van Dongen, E. Kindler, and C. W. Günther "Process mining: a two-step approach to balance between underfitting and overfitting," Softw Syst Model vol. 9, no. 87, 2010.

[59] P. Domingos, “A few useful things to know about machine learning," Commun. ACM, vol. 55, no. 10, pp. 78-87, 2012.

[60] Tibshirani, Robert, "Regression Shrinkage and Selection via the lasso," Journal of the Royal Statistical Society. Series B (methodological). Wiley, vol. 58, no. 1, pp. 267-88, 1996.

[61] Wikipedia contributors, "Minkowski distance". Wikipedia, The Free Encyclopedia. [Online]. Available: https://en.wikipedia.org. [Accessed: Feb.10, 2020]

[62] H. Drucker, C. C. Burges, L. Kaufman, A. J. Smola, and V. N. Vapnik, "Support Vector Regression Machines," in Advances in Neural Information Processing Systems 9, pp.155-161, 1996.

[63] C. Cortes and V. N. Vapnik "Support-vector networks," Machine Learning, vol. 20 no. 3, pp. 273297, 1995.

[64] H. TK, "The Random Subspace Method for Constructing Decision Forests," IEEE Transactions on Pattern Analysis and Machine Intelligence, vol. 20, no.8, pp. 832-844, 1998.

[65] L. Li, “Classification and Regression Analysis with Decision Trees, ” Towards Data Science, May 2019, [Online]. Available: .Https://towardsdatascience.com/https-medium-com-lorrli- 
classification-and-regression-analysis-with-decision-trees-c43cdbc58054. [Accessed: Feb. 10, 2020].

[66] M. Claesen and B. De Moor, Hyperparameter Search in Machine Learning, The XI Metaheuristics International Conference, 2015.

[67] R. Ghawi and J. Pfeffer, "Efficient Hyperparameter Tuning with Grid Search for Text Categorization using KNN Approach with BM25 Similarity,” Open Computer Science, vol. 9, no. 1, pp. 160-180. 2019.

[68] J. Bergstra, and Y. Bengio, "Random Search for Hyper-Parameter Optimization," Journal of Machine Learning Research, vol. 13, pp. 281-305, 2012.

[69] J. Snoek, H. Larochelle, and R. P. Adams, "Practical Bayesian optimization of machine learning algorithms," in Proc. of the 25th International Conf. on Neural Information Processing Systems, vol. 2, pp. 2951-2959, 2012.

[70] P. A. Devijver and J. Kittler, Pattern Recognition: A Statistical Approach. London, GB: PrenticeHall, 1982.

[71] J. Awwalu and F. Ogwueleka, "On Holdout and Cross Validation: A Comparison between Neural Network and Support Vector Machine," International Journal of Trend in Research and Development, vol. 6, no.2, pp. 2394-9333, 2019.

[72] T. Fushiki, "Estimation of prediction error by using K-fold cross-validation," Statistics and Computing vol. 21, pp. 137-146, 2011.

[73] C. Sammut and G. I. Webb G.I. (eds.), "Leave-One-Out Cross-Validation", Encyclopedia of Machine Learning. Springer, Boston, MA, 2011.

[74] G. E. P. Box and G. M. Jenkins. Time Series Analysis: Forecasting and Control., Revised edition. Holden-Day: San Francisco, 1976. 
[75] R. J. Hyndman and G. Athanasopoulos, Forecasting: Principles and practice. Vic. Heathmont: OTexts, 2014.

[76] S. Hochreiter and J. Schmidhuber, "Long Short-Term Memory," Neural Computation, vol. 9, no. 8, pp.1735-1780, 1997.

[77] S. Dupond, "A thorough review on the current advance of neural network structures," Annual Reviews in Control, vol. 14, pp. 200-230, 2019.

[78] L. M. Camarinha-Matos, R. Almeida and J. OliveiraI (eds.) “Technological Innovation for Industry and Service Systems," 10th IFIP WG 5.5/SOCOLNET Advanced Doctoral Conference on Computing, Electrical and Industrial Systems, DoCEIS, Costa de Caparica, Portugal, 2019.

[79] N. Srivastava, G. Hinton, A. Krizhevsky, I. Sutskever, and R. Salakhutdinov, "Dropout: a simple way to prevent neural networks from overfitting”. Journal of Machine Learning Research, vol. 15, no. 1 pp. $1929-1958,2014$.

[80] R. G. D. Steel and J. H. Torrie, Principles and Procedures of Statistics with Special Reference to the Biological Sciences. McGraw Hill, 1960.

[81] F. Pedregosa, G. Varoquaux, A. Gramfort, V. Michel, B. Thirion and O. Grisel, "Scikit-learn: Machine learning in Python,” Journal of Machine Learning Research, pp. 2825-2830, 2011.

[82] T. E. Oliphant, A guide to NumPy (Vol. 1). Trelgol Publishing, USA, 2006.

[83] W. McKinney, "Data structures for statistical computing in python," in Proc. of the 9th Python in Science Conf., vol. 445, pp. 51-56., 2010.

[84] J. D. Hunter, "Matplotlib: A 2D graphics environment," in Computing in Science \& Engineering, vol. 9, no.3, pp. 90-95, May-June 2007. 